\title{
LUMINOUS INFRARED GALAXIES. III. MULTIPLE MERGER, EXTENDED MASSIVE STAR FORMATION, GALACTIC WIND, AND NUCLEAR INFLOW IN NGC $3256^{1}$
}

\author{
S. LíPARI ${ }^{2,3}$ R. DíAz, ${ }^{2,3}$ Y. TANiguChI, ${ }^{4}$ R. TERLEVICH, ${ }^{5}$ H. DOTTORI, ${ }^{3,6}$ AND G. CARRANZA ${ }^{2}$ \\ Received 2000 March 29; accepted 2000 April 26
}

\begin{abstract}
We report detailed evidence for multiple merger, extended massive star formation, galactic wind, and circular/noncircular motions in the luminous infrared galaxy NGC 3256, based on observations of highresolution imaging (Hubble Space Telescope, ESO NTT), and extensive spectroscopic data (more than 1000 spectra, collected at Estación Astrofísica de Bosque Alegre, Complejo Astronómico el Leoncito, Cerro Tololo InterAmerican Observatory, and IUE observatories).

We find in a detailed morphological study (resolution $\sim 15 \mathrm{pc}$ ) that the extended massive star formation process detected previously in NGC 3256 shows extended triple asymmetrical spiral arms $(r \sim 5$ $\mathrm{kpc})$, emanating from three different nuclei. The main optical nucleus shows a small spiral disk $(r \sim 500$ pc), which is a continuation of the external one and reaches the very nucleus. The core shows blue elongated structure $(50 \mathrm{pc} \times 25 \mathrm{pc})$ and harbors a blue stellar cluster candidate $(r \sim 8 \mathrm{pc})$. We discuss this complex morphology in the framework of an extended massive star formation driven by a multiple merger process (models of Hernquist et al. and Taniguchi et al.).

We study the kinematics of this system and present a detailed $\mathrm{H} \alpha$ velocity field for the central region $\left(40^{\prime \prime} \times 40^{\prime \prime} ; r_{\max } \sim 30^{\prime \prime} \sim 5 \mathrm{kpc}\right)$, with a spatial resolution of $1^{\prime \prime}$ and errors of $\pm 15 \mathrm{~km} \mathrm{~s}^{-1}$. The color and isovelocity maps show mainly (1) a kinematic center of circular motion with "spider" shape, located between the main optical nucleus and the close $\left(5^{\prime \prime}\right)$ mid-IR nucleus and (2) noncircular motions in the external parts. We obtained three "sinusoidal rotation curves" (from the $\mathrm{H} \alpha$ velocity field) around position angle (P.A.) $\sim 55^{\circ}, \sim 90^{\circ}$, and $\sim 130^{\circ}$. In the main optical nucleus we found a clear "outflow component" associated with galactic winds plus an "inflow radial motion." The outflow component was also detected in the central and external regions $(r \leq 5-6 \mathrm{kpc})$. The main axis of the inflow region (P.A. $\sim 80^{\circ}$ ) is practically perpendicular to the ouflow axis (at P.A. $\sim 160^{\circ}$ ).

We analyze in detail the physical conditions in the giant $\mathrm{H}$ II regions located in the asymmetric spiral arms, the two main optical nuclei, and the outflow component (using long-slit spectroscopy, plus standard models of photoionization, shocks, and starbursts). We present four detailed emission-line ratios $\left(\mathrm{N} \mathrm{II} / \mathrm{H} \alpha, \mathrm{S} \mathrm{II} / \mathrm{H} \alpha, \mathrm{S} \mathrm{II} / \mathrm{S}\right.$ II), and FWHM $(\mathrm{H} \alpha)$ maps for the central region $\left(30^{\prime \prime} \times 30^{\prime \prime} ; r_{\max } \sim 22^{\prime \prime} \sim 4 \mathrm{kpc}\right)$, with a spatial resolution of $1^{\prime \prime}$. In the central region $(r \sim 5-6 \mathrm{kpc})$ we detected that the nuclear starburst and the extended giant $\mathrm{H}$ II regions (in the spiral arms) have very similar properties, i.e., high metallicity and low-ionization spectra, with $T_{\text {eff }}=35,000 \mathrm{~K}$, solar abundance, a range of $T_{e} \sim 6000-7000 \mathrm{~K}$, and $N_{e} \sim 100-1000 \mathrm{~cm}^{-3}$. The nuclear and extended outflow shows properties typical of galactic wind/ shocks, associated with the nuclear starburst. We suggest that the interaction between dynamical effects, the galactic wind (outflow), low-energy cosmic rays, and the molecular+ionized gas (probably in the inflow phase) could be the possible mechanism that generate the "similar extended properties in the massive star formation, at a scale of 5-6 kpc!"

We have also studied the presence of the close merger/interacting systems NGC 3256C (at $\sim 150 \mathrm{kpc}$, $\Delta V=-100 \mathrm{~km} \mathrm{~s}^{-1}$ ) and the possible association between the NGC 3256 and 3263 groups of galaxies. In conclusion, these results suggest that NGC 3256 is the product of a multiple merger, which generated an extended massive star formation process with an associated galactic wind plus a nuclear inflow. Therefore, NGC 3256 is another example in which the relation between mergers and extreme starburst (and the powerful galactic wind, "multiple" Type II supernova explosions) play an important role in the evolution of galaxies (the hypothesis of Rieke et al., Joseph et al., Terlevich et al., Heckman et al., and Lípari et al.).
\end{abstract}

Key words: galaxies: evolution - galaxies: individual (NGC 3256, NGC 3256C, NGC 3256A, NGC 3256B, NGC 3263, NGC 3262) - galaxies: interactions -

galaxies: kinematics and dynamics - galaxies: starburst - infrared radiation quasars: general

\footnotetext{
${ }^{1}$ Based on observations obtained at the Hubble Space Telescope (HST; Wide Field Planetary Camera 2 [WFPC2] and NICMOS) satellite; International Ultraviolet Explorer (IUE) satellite; European Southern Observatory (ESO, NTT); Chile, Cerro Tololo Inter-American Observatory (CTIO), Chile; Complejo Astronómico el Leoncito (CASLEO), Argentina; Estación Astrofísica de Bosque Alegre (BALEGRE), Argentina.

${ }^{2}$ Cordoba Observatory and CONICET, Laprida 854, 5000 Cordoba, Argentina; lipari@mail.oac.uncor.edu.

${ }^{3}$ Visiting Astronomer, BALEGRE, CASLEO, CTIO, and ESO observatories.

${ }^{4}$ Astronomical Institute, Tohoku University, Aoba, Sendai 980-8578, Japan.

${ }^{5}$ Institute of Astronomy, Madingley Road, Cambridge CB3 0HA, England, UK.

${ }^{6}$ Instituto de Fisica, Univ. Fed. Rio Grande do Sul, CP 15051, Porto Alegre, Brazil.
} 


\section{INTRODUCTION}

An important current issue in astrophysics and cosmology (especially at high redshift) is the study of star formation in merger processes and their relation to the evolution and formation of galaxies (see, for references, Djorgovski 1994; White 1994; Weil \& Hernquist 1996; Scott et al. 1998). Furthermore, this issue is also related to the detection of highly energetic activity in the nuclei of galaxies (see Toomre \& Toomre 1972; Toomre 1977; Rees 1984; Joseph \& Wright 1985; Sanders et al. 1988a, 1988b; Norman \& Scoville 1988; Taniguchi, Ikeuchi, \& Shioya 1999). On the other hand, detailed studies of nearby mergers give important clues to understanding properly high-redshift mergers and merger candidates.

Luminous infrared galaxies (LIRGs; $L_{\mathrm{IR}} \geq 10^{11} L_{\odot}$ ) are dusty strong IR emitters $\left(L_{\mathrm{IR}} / L_{B} \sim 10-300\right)$, where frequently an enhancement of star formation is taking place (see Rieke \& Low 1972, 1975; Rieke et al. 1980, 1985; Weedman et al. 1981; Joseph \& Wright 1985; Norris et al. 1990; Heckman, Armus, \& Miley 1990, hereafter HAM90; Hutching \& Neff 1991; Condon et al. 1991a, 1991b; Lípari, Terlevich, \& Macchetto 1993a; Lutz et al. 1996; Downes \& Solomon 1998). Furthermore, in LIRGs, "galactic wind/ bubble" features originating in massive stars and supernova (SN) explosions have been also detected (Ulrich 1972, 1978; Heckman, Armus, \& Miley 1987; HAM90; McCarthy, Heckman, \& van Breugel 1987; Bland \& Tully 1988; Taniguchi, Trentham, \& Shioya 1988; Fabbiano 1988; Colina, Lípari, \& Macchetto 1991; Filippenko \& Sargent 1992; Phillips 1993; Lípari, Colina, \& Macchetto 1994; Veilleux et al. 1994; Lípari, Tsvetanov, \& Macchetto 1997). However, at high IR luminosities, there is also an increase in the nonthermal nuclear activity (Sanders et al. 1988a; Veilleux et al. 1996; Sanders \& Mirabel 1996; Veilleux, Kim, \& Sanders 1999).

On the other hand, imaging surveys of ultraluminous IR galaxies (ULIRGs; $L_{\mathrm{IR}} \geq 10^{12} L_{\odot}$ ) show that $\sim 100 \%$ are mergers or interacting systems (Sanders et al. 1988a; Melnick \& Mirabel 1990; Clements et al. 1996; Joseph \& Wright 1985; Rieke et al. 1985). In addition, virtually all LIRGs have been shown to be extremely rich in interstellar molecular gas (Sanders, Scoville, \& Soifer 1991) and highly concentrated in their nuclei (Scoville et al. 1991; Okumura et al. 1991; Downes \& Solomon 1998). This matter reservoir plays a central role in promoting star formation and probably fueling a massive compact object (Scoville \& Soifer 1991).

Mergers are mainly luminous IR galaxies in which their luminosities overlap with most luminous quasistellar objects (QSOs) and Seyfert galaxies; and their optical, IR, and radio properties show mainly starburst and active galactic nucleus (AGN) characteristics (Joseph \& Wright 1985; Schweizer 1980, 1982, 1990; Rieke et al. 1985; Heckman et al. 1987; Sanders et al. 1988a; Colina et al. 1991; Lípari et al. 1994; Veilleux et al. 1996, 1999; Lutz et al. 1996; Genzel et al. 1998). In addition, there is compelling evidence for merger- or interaction-driven starburst and nuclear activity, probably by depositing large amount of interstellar gas to the central regions (see Sanders \& Mirabel 1996; Scoville \& Soifer 1991; Barnes \& Hernquist 1992; Mihos \& Hernquist 1996, 1994a, 1994b). Furthermore, there are also strong evidences for galactic wind features, from young starbursts in luminous IR mergers (see
$\S$ 4.5). Also, several possible links between mergers, starburst, IR galaxies/QSOs, and evolution of galaxies have been proposed (see, for references, Lípari et al. 2000a).

NGC 3256 is a nearby luminous IR galaxy $(z=0.0094$; $\S 3.2)$ that has been thought to be a major merger between two gas-rich galaxies (Toomre 1977; Graham et al. 1984; Joseph \& Wright 1985; Schweizer 1986, 1990; Joseph 1991; Moorwood \& Oliva 1994; Sanders et al. 1995). This system shows knotty circumnuclear structure (Sersic 1959) and two extended tidal tails (de Vaucouleurs \& de Vaucouleurs 1961). The total IR luminosity of NGC 3256 is $L_{\mathrm{IR}[8-1000 \mu \mathrm{m}]}=3.3 \times 10^{11} L_{\odot}$; consequently, the bolometric luminosity makes this system the most luminous nearby galaxy (for $V \leq 3000 \mathrm{~km} \mathrm{~s}^{-1}$; Sargent, Sanders, \& Phillips 1989).

The strong $10 \mu \mathrm{m}$ IR emission in NGC 3256 is "very extended," with most of it originating outside the central kiloparsecs, indicating clearly that the near-/mid-IR emission is probably powered by a recent starburst and not by a compact object or an AGN (Graham et al. 1984; Joseph \& Wright 1985; Joseph 1991). In addition, half of molecular hydrogen mapped in $\mathrm{CO}\left(1.5 \times 10^{10} M_{\odot} \mathrm{H}_{2}\right)$ is located outside of the central $2.5 \mathrm{kpc}$ (Sargent et al. 1989). NGC 3256 is also the galaxy with the more extended polarized system known (Scarrott, Draper, \& Stockdale 1996). The extended nature at near-/mid-IR and $\mathrm{CO}$ emission confirms that NGC 3256 is not a very advanced merger.

Throughout the paper, a Hubble constant of $H_{0}=75 \mathrm{~km}$ $\mathrm{s}^{-1} \mathrm{Mpc}^{-1}$ will be assumed. For NGC 3256 we adopted the distance of $\sim 37 \mathrm{Mpc}\left(V_{\text {syst }}=2817 \pm 15 \mathrm{~km} \mathrm{~s}^{-1} ; \S 3.2 .1\right)$, and thus, the angular scale is $1^{\prime \prime} \approx 177 \mathrm{pc}$.

\section{OBSERVATIONS AND REDUCTIONS}

The optical and near-IR observations were obtained at BALEGRE, CASLEO, CTIO, and ESO Observatories with the 1.54, 2.15, 1.0, and $3.5 \mathrm{~m}$ telescopes, respectively. In addition, HST WFPC2 and NICMOS and ESO NTTSuper Seeing Imager (SUSI) archive images of NGC 3256 were studied.

The HST WFPC2 observations include broadband images, using the filters F450W ( $\sim B$ Cousins filter) and F814W $(\sim I)$, with a CCD scale of 0.046 pixel $^{-1}$, in the planetary camera (PC). HST NICMOS observations used the NIC2 and NIC1 camera, with the filters F160W (1.60 $\mu \mathrm{m}$, wide), F187N (1.87 $\mu \mathrm{m}$, narrow), F190N $(1.90 \mu \mathrm{m})$, F212N $(2.12 \mu \mathrm{m})$, F215N $(2.15 \mu \mathrm{m})$, F222M $(2.22 \mu \mathrm{m}$, medium), and F237M (2.37 $\mu \mathrm{m})$ (Table 1).

The ESO NTT data include $V$ broadband images (ESO Multi-Mode Instrument [EMMI]), and [O III] $\lambda \lambda 4959+5007$ and $\mathrm{H} \alpha+[\mathrm{N}$ II $] \lambda \lambda 6548+6584$ narrowband images (SUSI). The NTT SUSI and EMMI data (Table 1) have seeings of 0 ."7 and 1"4 FWHM and CCD scales of 0.13 and 0 .44 pixel $^{-1}$, respectively.

Moderate-resolution long-slit spectrophotometric observations were obtained at the $1.0 \mathrm{~m}$ telescope of CTIO during two photometric nights in 1990 March (Table 1). The observations were made using a slit width of 1.15 , which gives an effective resolution of $\sim 8 \AA\left(\sim 300 \mathrm{~km} \mathrm{~s}^{-1}\right)$, at the wavelength range $\lambda \lambda 3600-6900$. The seeing was approximately 1 1.5.

Images and spectrophotometric observations of NGC 3256 were taken at CASLEO, San Juan, Argentina. Longslit spectroscopic observations with moderate resolution 
TABLE 1

Journal of ObSERVATIONS OF NGC 3256 (AND the NGC 3256 Group of GalaXIES)

\begin{tabular}{|c|c|c|c|}
\hline Date & Telescope/Instrument & Spectral Region & Comments \\
\hline 1990 Mar $03 . . . .$. & CTIO $1.0 \mathrm{~m}$ & $\lambda \lambda 3600-6900$ & P.A. $90^{\circ}$ long-slit $=1^{\prime \prime} .5$ \\
\hline 1993 Feb $22 \ldots \ldots$. & ESO EMMI $3.5 \mathrm{~m}$ & $V$ & Images \\
\hline 1993 Apr 09 ...... & ESO SUSI $3.5 \mathrm{~m}$ & $\begin{array}{l}\lambda \lambda 5013 / 139 \AA \\
\lambda \lambda 5111 / 55 \AA \\
\lambda \lambda 6571 / 115 \AA \\
\lambda \lambda 7018 / 65 \AA\end{array}$ & $\begin{array}{l}{[\mathrm{O} \text { III] redshifted }} \\
{[\mathrm{O} \text { III }] \text { continuum }} \\
\mathrm{H} \alpha+[\mathrm{N} \text { II }] \text { redshifted } \\
\mathrm{H} \alpha+[\mathrm{N} \text { II }] \text { continuum }\end{array}$ \\
\hline 1994 Sep $7 \ldots \ldots \ldots$ & $H S T$ WFPC2 & $\begin{array}{l}\text { F814W, } \lambda \lambda 7970 / 1531 \AA \\
\text { F450W, } \lambda \lambda 4521 / 958 \AA\end{array}$ & \\
\hline 1997 Dec $5 \ldots \ldots \ldots$ & HST NICMOS & $\begin{array}{l}\mathrm{F} 160 \mathrm{~W}, \lambda \lambda 1.60 / 0.20 \mu \mathrm{m} \\
\mathrm{F} 110 \mathrm{M}, \lambda \lambda 1.10 / 0.10 \mu \mathrm{m} \\
\mathrm{F} 222 \mathrm{M}, \lambda \lambda 2.22 / 0.07 \mu \mathrm{m} \\
\mathrm{F} 237 \mathrm{M}, \lambda \lambda 2.37 / 0.07 \mu \mathrm{m} \\
\mathrm{F} 166 \mathrm{~N}, \lambda \lambda 1.66 / 0.01 \mu \mathrm{m} \\
\mathrm{F} 190 \mathrm{~N}, \lambda \lambda 1.90 / 0.01 \mu \mathrm{m} \\
\text { F215N, } \lambda \lambda 2.15 / 0.01 \mu \mathrm{m}\end{array}$ & $\begin{array}{l}\text { NIC2 } \\
\text { NIC1 } \\
\text { NIC2 } \\
\text { NIC2 } \\
\text { NIC1 } \\
\text { NIC2 } \\
\text { NIC2 }\end{array}$ \\
\hline 1997 Mar $11 \ldots . .$. & CASLEO $2.15 \mathrm{~m}$ & $\begin{array}{l}B \\
V \\
I\end{array}$ & Images \\
\hline 1997 Mar $12 \ldots . .$. & & $\lambda \lambda 4100-7500$ & P.A. $90^{\circ}$ long-slit $=2^{\prime \prime}$ \\
\hline 1997 Apr 30 ..... & BALEGRE $1.5 \mathrm{~m}$ & $\lambda \lambda 6400-6900$ & P.A. $80^{\circ}$ long-slit \\
\hline 1997 May $5 . . . . .$. & EMF & $\lambda \lambda 6400-6900$ & P.A. $110^{\circ}, 80^{\circ}, 00^{\circ}$ slit $=1^{\prime \prime}$ \\
\hline 1997 May $6 . . . . .$. & & $\lambda \lambda 6400-6900$ & P.A. $90^{\circ}, 00^{\circ}, 110^{\circ}$ \\
\hline 1997 May 7 ...... & & $\lambda \lambda 6400-6900$ & P.A. $135^{\circ}, 45^{\circ}$ \\
\hline 1997 May 8 ...... & & $\lambda \lambda 6400-6900$ & P.A. $25^{\circ}, 155^{\circ}$ \\
\hline 1997 May $9 . . . .$. & & $\lambda \lambda 6400-6900$ & P.A. $65^{\circ}, 110^{\circ}$ \\
\hline 1997 May $11 \ldots . .$. & & $\lambda \lambda 6400-6900$ & P.A. $25^{\circ}, 125^{\circ}$ \\
\hline 1997 May $12 \ldots \ldots$ & & $\lambda \lambda 6400-6900$ & P.A. $45^{\circ}$ \\
\hline 1997 Jun 6 ........ & & $\lambda \lambda 6400-6900$ & P.A. $56^{\circ}, 168^{\circ}$ \\
\hline \multirow{2}{*}{1997 Jun 7 ........... } & & $\lambda \lambda 6400-6900$ & P.A. $145^{\circ}$ \\
\hline & & $\lambda \lambda 4800-6900$ & P.A. $90^{\circ} 7^{\prime \prime} \mathrm{S}$ \\
\hline \multirow[t]{2}{*}{1997 Jun $8 \ldots \ldots \ldots$} & & $\lambda \lambda 6400-6800$ & P.A. $13^{\circ}$ \\
\hline & & $\lambda \lambda 4800-6900$ & P.A. $90^{\circ} 7^{\prime \prime} \mathrm{S}$ \\
\hline 1997 Jun $9 \ldots \ldots$. & & $\lambda \lambda 6400-6900$ & P.A. $100^{\circ}$ \\
\hline $1997 \mathrm{Jul} 4$......... & & $\lambda \lambda 6400-6900$ & P.A. $40^{\circ}$ \\
\hline 1997 Jul 5 ........ & & $\lambda \lambda 6400-6900$ & P.A. $130^{\circ}, 40^{\circ}$ \\
\hline 1997 Jul $13 \ldots \ldots \ldots$ & & $\lambda \lambda 6400-6900$ & P.A. $90^{\circ} 7^{\prime \prime} \mathrm{S}$ \\
\hline 1997 Jul $15 \ldots \ldots \ldots$ & & $\lambda \lambda 6400-6900$ & P.A. $90^{\circ} 7^{\prime \prime} \mathrm{S}$ \\
\hline \multirow[t]{2}{*}{1998 Feb $26 \ldots \ldots$} & & $\lambda \lambda 6400-6900, V, I$ & P.A. $90^{\circ}$ (N3256C, N3261) \\
\hline & & $\lambda \lambda 6400-6900, V, I$ & P.A. $108^{\circ}(\mathrm{N} 3263)$ \\
\hline \multirow[t]{2}{*}{1998 Feb $27 \ldots \ldots$} & & $\lambda \lambda 6400-6900$ & P.A. $18^{\circ}$ \\
\hline & & $\lambda \lambda 6400-6900$ & P.A. $82^{\circ}$ tail NE $48^{\prime \prime} \mathrm{N}$ \\
\hline 1998 May $29 . . . .$. & & $\lambda \lambda 6400-6900$ & P.A. $70^{\circ}$ \\
\hline 1998 May $30 \ldots . .$. & & $\lambda \lambda 6400-6900$ & P.A. $50^{\circ}$ tail NE $33^{\prime \prime} \mathrm{N}$ \\
\hline 1999 Jan $14 \ldots \ldots$. & & $\lambda \lambda 6400-6900, V, I$ & P.A. $90^{\circ}(\mathrm{N} 3262)$ \\
\hline 1999 Feb $19 \ldots \ldots$ & & $\lambda \lambda 6400-6900, V, I$ & P.A. $67^{\circ}(\mathrm{N} 3256 \mathrm{C})$ \\
\hline 1999 Mar $22 \ldots \ldots$ & & $\lambda \lambda 6400-6900$ & P.A. $90^{\circ}, 137^{\circ}(\mathrm{N} 3256 \mathrm{~A} / 3256 \mathrm{~B})$ \\
\hline 1999 Mar $23 \ldots \ldots$. & & $V, I$ & N3256A, B, C; N3261/3262/3263 \\
\hline
\end{tabular}

were obtained with the University of Columbia spectrograph (Lípari et al. 1997) at the $2.15 \mathrm{~m}$ Ritchey-Chrétien telescope of CASLEO during two photometric nights in 1997 March (see Table 1). The observations were made using a slit width of 2.0 , which gives an effective resolution of $\sim 7 \AA\left(\sim 280 \mathrm{~km} \mathrm{~s}^{-1}\right)$, at the wavelength range $\lambda \lambda 4100$ 7500. Broadband CCD imaging observations were obtained in 1997 March (see Table 1). Images through $B, V$, and $I$ filters were also obtained with a TektroniX $1 \mathrm{~K}$ chip with a scale of $0.813 \mathrm{pixel}^{-1}$. The observing conditions were photometric, and the seeing was $~ 1 " .8-2 " .5$.

Extensive long-slit spectroscopic observations were obtained at the $1.54 \mathrm{~m}$ telescope of Bosque Alegre Station of Cordoba Observatory using the Multifunctional Integral Field Spectrograph (Afanasiev, Dodonov, \& Carranza
1994; Diaz et al. 1999) during 18 nights between 1997 April and 1999 March (Table 1). The observations were made mainly using a slit width of $1^{\prime \prime} .0$ and a 1200 line $\mathrm{mm}^{-1}$ grating, which gives an effective resolution of $\sim 90 \mathrm{~km} \mathrm{~s}^{-1}$ and covering the wavelength range $\lambda \lambda 6400-6900$. In order to have accurate spatial positions for the velocity determinations in each extracted spectrum, zero-order double imaging of object plus slit before and after the spectroscopic exposures were used. Spectrophotometric observations were also obtained using a 300 line $\mathrm{mm}^{-1}$ grating, with an effective resolution of $\sim 330 \mathrm{~km} \mathrm{~s}^{-1}$, and covering the wavelength range $\lambda \lambda 4800-6900$. The seeing was approximately 1".4-2".5 (FWHM). A Thomson $1 \mathrm{~K}$ chip with a scale of 0.38 pixel $^{-1}$ was used. The direct image mode gives a field of $5.7 \times 5.7$. 
UV-IUE/Short Wavelength Prime (SWP) and near-IR archive spectra (covering $\lambda \lambda 1200-2000$ and $\lambda \lambda 7000-10000$, with resolutions of $\sim 8$ and $5 \AA$, respectively) were obtained from the Starburst Galaxies Spectra Catalog of StorchiBergmann, Kinney, \& Challis (1995). The IUE spectra were obtained with a wide aperture of $10^{\prime \prime} \times 20^{\prime \prime}$, and in the near-IR long-slit data, the slit width was $10^{\prime \prime}$ and the extracted window $20^{\prime \prime}$ (in order to get a final aperture close to the IUE observations).

The IRAF ${ }^{7}$ software packages were used to reduce the imaging and spectrophotometric data (obtained at CTIO and CASLEO). Bias, dark subtraction, and flat-fielding were performed in the usual way. Wavelength calibration of the spectra was done by fitting two-dimensional polynomials to the position of lines in the arc frame. The spectra were corrected for atmospheric extinction, galactic reddening, and redshift. The spectra and images were flux calibrated using observations of standard stars from the samples of Stone \& Baldwin (1983) and Landolt (1992). The $H S T$ archive data were obtained with the best reduction performed (at the Space Science Telescope Institute [STScI]).

The kinematical observations obtained at BALEGRE (more than 25 position angles of long-slit frames-mainly centered in the main optical nucleus - from which were extracted $\sim 1000$ individual spectra of 0.76 ) were reduced and analyzed using mainly $\mathrm{SAO}^{8}$ and $\mathrm{ADHOC}^{9}$ software packages (see Diaz et al. 1999 for references and the velocity field reduction). The emission lines were measured and decomposed using Gaussian profiles by means of a nonlinear least-squares algorithm described in Bevington (1969).

\section{RESULTS}

\subsection{Optical and Near-IR Structures}

A CASLEO $B$ broadband image, for the whole merger, is displayed in Figure 1a. An ESO NTT-EMMI $V$ broadband

\footnotetext{
${ }^{7}$ IRAF is distributed by the National Optical Astronomy Observatories, which are operated by the Association of Universities for Research in Astronomy, Inc., under cooperative agreement with the National Science Foundation.

${ }^{8}$ Imaging analysis software developed by the Special Astrophysical Observatory of the USSR Academy of Science.

${ }^{9}$ Imaging analysis software developed by Marseille Observatory.
}

image of the central and external regions of NGC 3256 is shown in Figure $1 b$. An ESO NTT-SUSI high-resolution narrowband image in $\mathrm{H} \alpha+[\mathrm{N}$ II $] \lambda \lambda 6548+6584$ of the central area is presented in Figure $1 c$ (the values of the seeing were $1 . .8,1$.".4, and 0.7 , respectively).

HST WFPC2 and NICMOS broad/mid-band and color images are presented in Figures $1 d, 1 e, 1 f$, and $1 g$ (for the filters F814W-PC2, F450W/F814W-PC2, and F237MNIC2).

This galaxy consists of a main body $\left(r \sim 9 \mathrm{kpc} \sim 60^{\prime \prime}\right)$, two extended tidal tails $(\sim 80 \mathrm{kpc})$, and two faint external loops. In the central $\left(r \sim 5 \mathrm{kpc} \sim 30^{\prime \prime}\right)$ and nuclear $(r \sim 2$ $\mathrm{kpc} \sim 10^{\prime \prime}$ ) regions, several bright optical and IR knots are the prominent structures. The general properties of NGC 3256 and luminous IR ongoing mergers are presented in Table 2.

\subsubsection{The Nuclear and Central Regions $\left(r \leq 30^{\prime \prime} \sim 5 \mathrm{kpc}\right)$}

The central regions of NGC 3256 show a distorted ellipsoid shape with a southwest extension (Figs. $1 b, 1 c, 1 d$, and 1e). The ESO NTT-SUSI and HST WFPC2 images (Figs. $1 c, 1 d$, and $1 e$ ) show "together" that inside this ellipsoid there is a bright knotty structure that shows a very unusual triple asymmetrical spiral arm morphology, which projected in the sky - at low resolution-looks like rings or "chaotic" knotty structures (Sersic 1959).

These three asymmetrical spiral arms (I, II, and III; see Fig. 1c) start in the three main nuclear knots (Figs. 1d, 1e, and $1 g$ ). Arm I (which probably emanates from the main optical nucleus; Fig. 1e) shows the more extended morphology and a secondary arm in the north area. Arm II, probably the nearest arm, shows strong blue colors (Table 3) and seems to be connected to the optical blue knot located at 5.7 to the east of the main optical nucleus. Arm III is partially obscured by dust and is probably connected to the mid-IR knot located at $5^{\prime \prime}$ to the south from the main optical nucleus (Fig. 1g). In the areas of arms intersection, Figures $1 c, 1 d$, and $1 e$ show clearly that (1) arm II is behind arm I and (2) arm III is behind arm II. Therefore, these arms are in three different planes.

The most prominent compact optical and near-IR knots (Figs. 1c, 1d, and 1i) are (1) the stronger optical knot or the main optical nucleus, that we call region 1 (hereafter R1); (2)

TABLE 2

General Properties of NGC 3256 and Luminous IR Ongoing Mergers

\begin{tabular}{|c|c|c|c|c|c|c|c|}
\hline Parameters & N4038/4039 & N3256 & A220 & N6240 & N7252 & Mk 231 & I19254-7245 \\
\hline \multicolumn{8}{|l|}{ Parameters adopted: } \\
\hline$D\left(\mathrm{Mpc}, H_{0}=75\right) \ldots \ldots \ldots \ldots \ldots \ldots \ldots \ldots \ldots \ldots \ldots$ & 22 & 35 & 77 & 98 & 64 & 170 & 236 \\
\hline Inclination $(\mathrm{deg}) \ldots \ldots \ldots \ldots \ldots \ldots \ldots$ & $\ldots$ & $40-45$ & $\ldots$ & $\ldots$ & 41 & $\ldots$ & $20-30$ \\
\hline \multicolumn{8}{|l|}{ Parameters derived: } \\
\hline Systemic velocity $\left(\mathrm{km} \mathrm{s}^{-1}\right) \ldots \ldots \ldots \ldots$ & 1650 & 2817 & 5450 & 7300 & 4800 & 12,450 & 17,900 \\
\hline 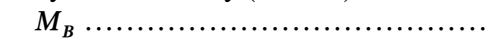 & -22.4 & -22.6 & -20.8 & -21.5 & -21.2 & -22.3 & -23.3 \\
\hline $\log L_{\mathrm{IR}}\left(L_{\odot}\right) \ldots \ldots \ldots \ldots \ldots \ldots \ldots \ldots \ldots \ldots \ldots \ldots \ldots \ldots \ldots$ & $\sim 11$ & 11.5 & 12.2 & 11.8 & 10.5 & 12.5 & 12.1 \\
\hline$L_{\mathrm{IR}} / L_{B} \ldots \ldots \ldots \ldots \ldots \ldots \ldots \ldots \ldots \ldots \ldots \ldots \ldots \ldots \ldots \ldots \ldots \ldots$ & 1 & 50 & 150 & 45 & 1 & 300 & 30 \\
\hline $\operatorname{SFR}\left(M_{\odot} \mathrm{yr}^{-1}\right) \ldots \ldots \ldots \ldots \ldots \ldots$ & 5 & 80 & 300 & 100 & 3 & $\sim 1000$ & $150-500$ \\
\hline SN rate $\left(\mathrm{yr}^{-1}\right) \ldots \ldots \ldots \ldots \ldots \ldots \ldots \ldots$ & 0.1 & 2 & 3 & 1.0 & 0.05 & 5 & 4 \\
\hline$d_{\text {nucl }}\left(\mathrm{kpc}, H_{0}=75\right) \ldots \ldots \ldots \ldots \ldots \ldots$ & 6.4 & 1.0 & 0.4 & 0.5 & 0.0 & 0.0 & 10.0 \\
\hline 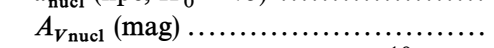 & 70 & 35 & $100-1000$ & $20-40$ & $\sim 1$ & $\geq 20$ & $\geq 10$ \\
\hline Dynam. total mass $\left(M_{\odot} \times 10^{10}\right) \ldots \ldots$ & 8.0 & $\sim 5$ & $\sim 1$ & $(8)$ & 4.0 & $\ldots$ & $\ldots$ \\
\hline Molec. $\mathrm{H}_{2}$ mass $\left(M_{\odot} \times 10^{9}\right) \ldots \ldots \ldots \ldots$ & 5.0 & 30 & 60.3 & 20.0 & 3.6 & 58.9 & 3.0 \\
\hline Atomic H I mass $\left(M_{\odot} \times 10^{9}\right) \ldots \ldots \ldots$ & 9.8 & $\ldots$ & $\ldots$ & $\ldots$ & 3.4 & $\leq 22.4$ & $\ldots$ \\
\hline Ionized $\mathrm{H}$ II mass $\left(M_{\odot} \times 10^{7}\right) \ldots \ldots \ldots$ & $\ldots$ & $\ldots$ & $\ldots$ & $\ldots$ & 11 & $\ldots$ & 11 \\
\hline Dust mass $\left(M_{\odot} \times 10^{7}\right) \ldots \ldots \ldots \ldots \ldots$ & 1.4 & $(2.0)$ & 12.0 & 7.0 & $\leq 1.5$ & 12.0 & 1.5 \\
\hline
\end{tabular}




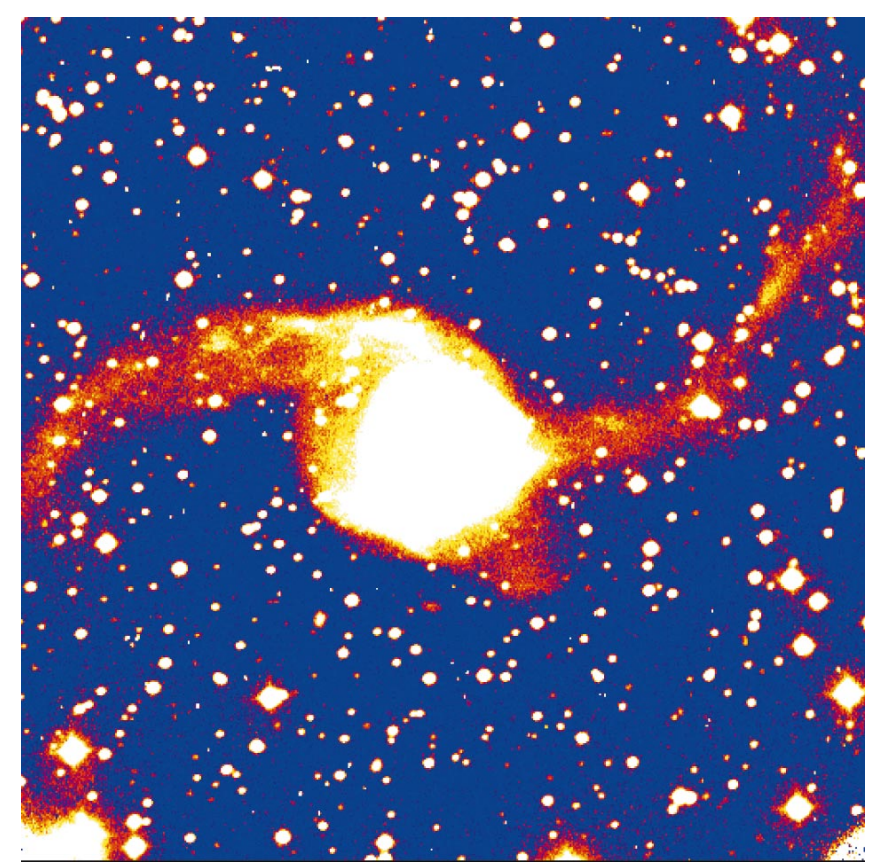

FIG. $1 a$

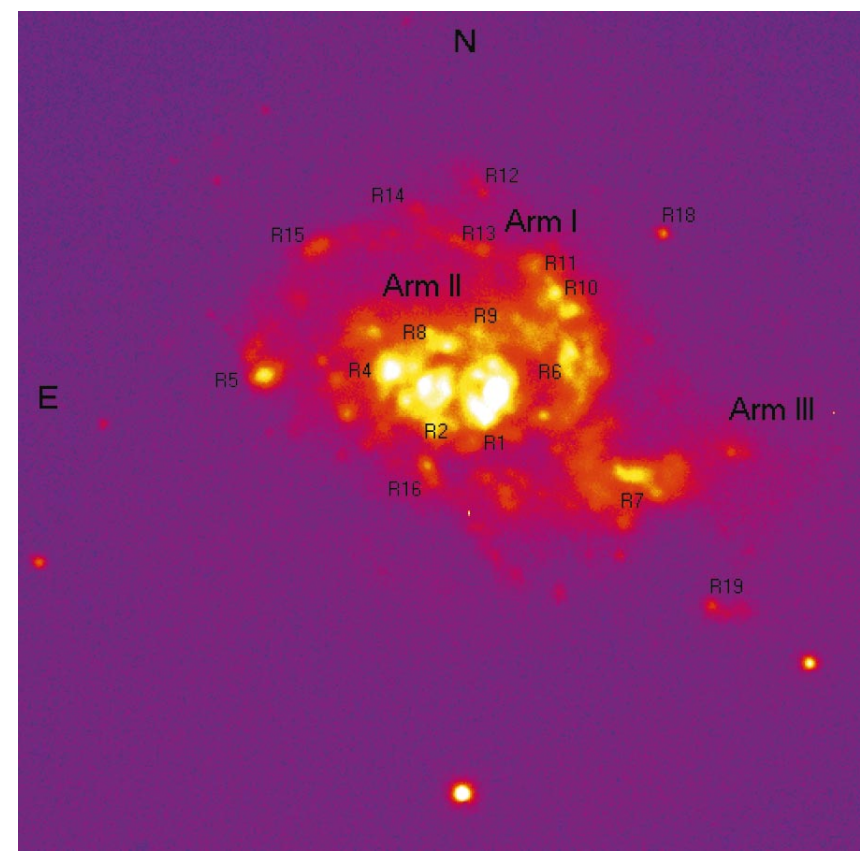

FIG. 1c

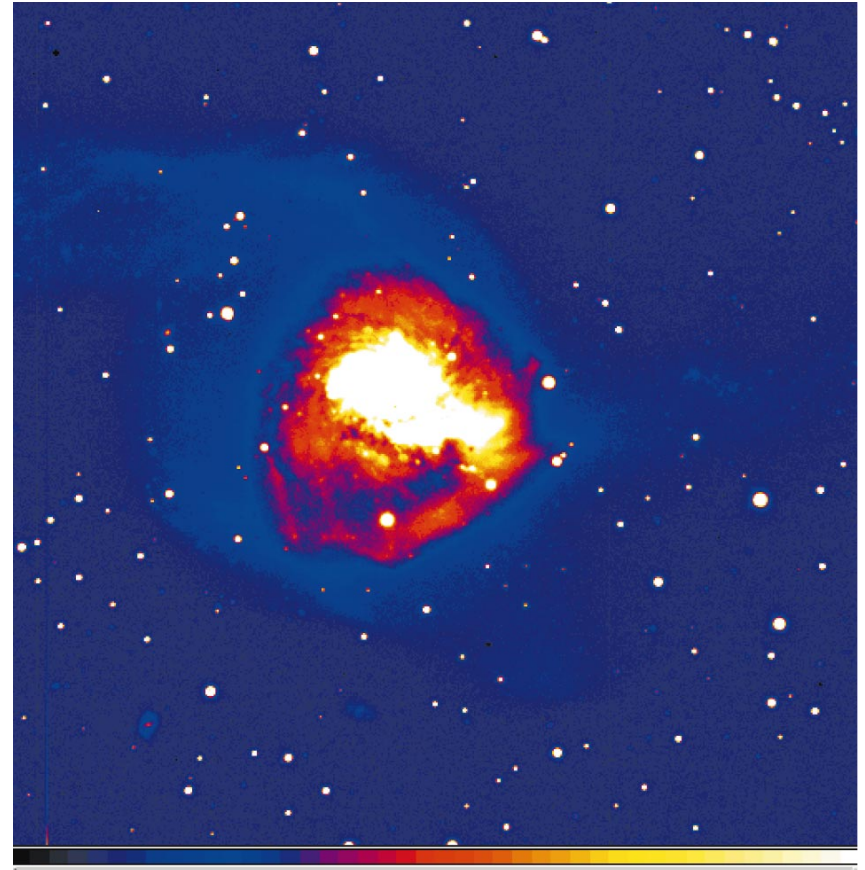

FIG. $1 b$

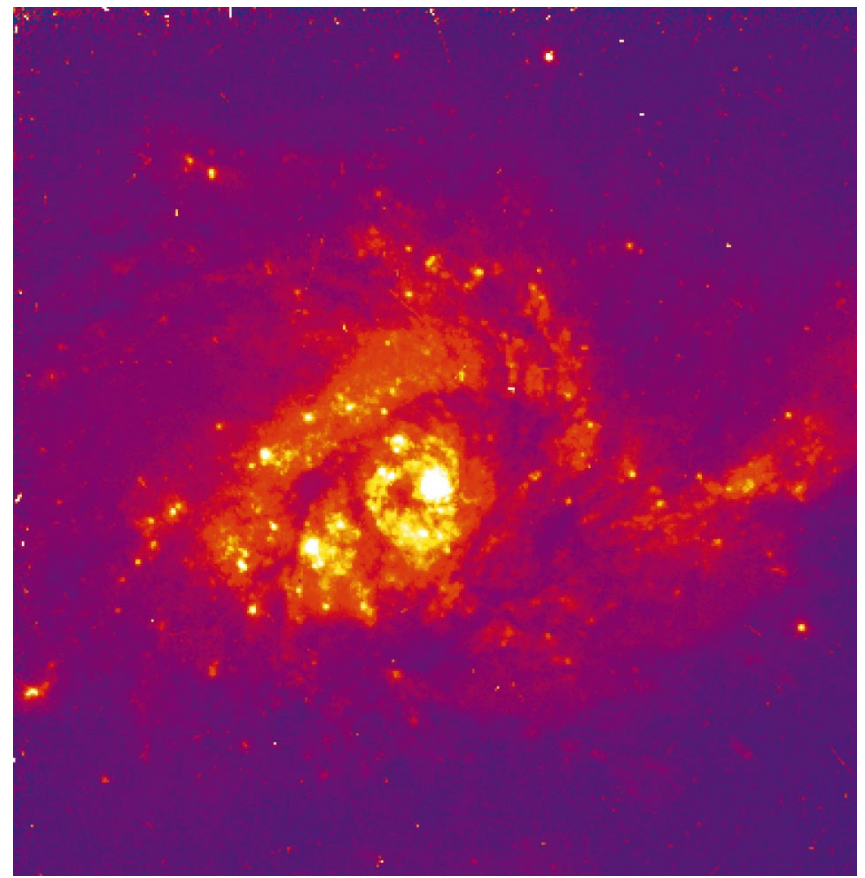

FIG. $1 d$

FIG. 1. - CCD broad- and narrowband images of NGC 3256. North is up, and east is to the left (in the HST WFPC2 and NICMOS images the north-south lines are rotated $\sim 35^{\circ}$ and $\sim 30^{\circ}$ to the left, respectively). (a) $B$ CASLEO image $\left(\sim 2^{\prime} \times 2^{\prime}\right.$, seeing 1'.8. (b) $V$ NTT-EMMI image $\left(\sim 1^{\prime} .5 \times 11^{\prime} .5\right.$, seeing 1"4). (c) Pure $\mathrm{H} \alpha+[\mathrm{N} \mathrm{II}] \lambda \lambda 6548,6583$ NTT-SUSI images $\left(\sim 1^{\prime} \times 1^{\prime}\right.$, seeing 0"7). (d) HST WFPC2 F814W image $\left(\sim 40^{\prime \prime} \times 40^{\prime \prime}\right.$, point-spread function 0"1). (e) Central region of $(d)$ (WFPC2 F814W image, $\left.\sim 30^{\prime \prime} \times 30^{\prime \prime}\right) .(f) H S T$ WFPC2 F450W/F814W image $\left(\sim 10^{\prime \prime} \times 10^{\prime \prime}\right)$; the yellow false color shows high values of the ratio F450W/F814W. $(g)$ HST NICMOS F222M image $\left(\sim 19^{\prime \prime} \times 19^{\prime \prime}\right)$. (h)-(i) HST WFPC2 F450W image contour $\left(1{ }^{\prime \prime} 5 \times 11^{\prime \prime} 5\right)$, for the main optical nucleus (R1) and R2.

a similar compact knot located $5.7(\sim 1 \mathrm{kpc})$ to the east from the optical nucleus (region 2, hereafter R2); and (3) an obscured knot detected only at wavelengths $\lambda \geq 3.75 \mu \mathrm{m}$ (region 3, hereafter R3). These three knots are probably the nuclei of the original galaxies that collided (Zenner \&
Lenzen 1993; Moorwood \& Oliva 1994; Norris \& Forbes 1995; Kotilainen et al. 1995).

In particular, R1 and R2 show giant $\mathrm{H}$ II region properties with blue colors and starburst spectra (see $\S 3.3$ ). The [O III] $\lambda \lambda 4959-5007$ and $B$-band images show that R2 


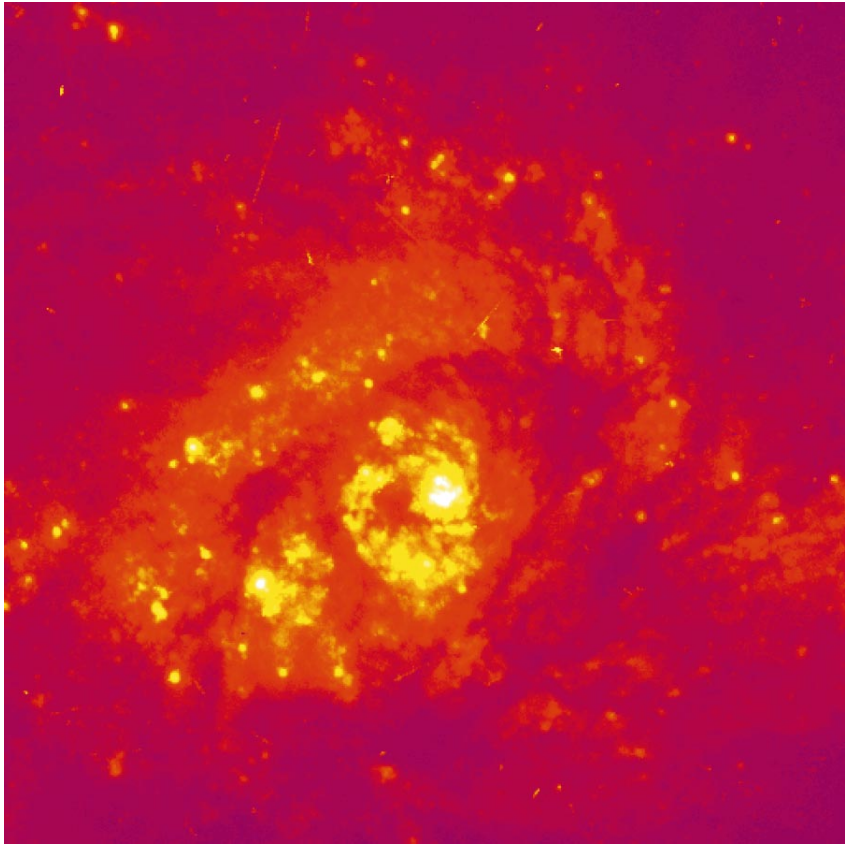

FIG. 1e

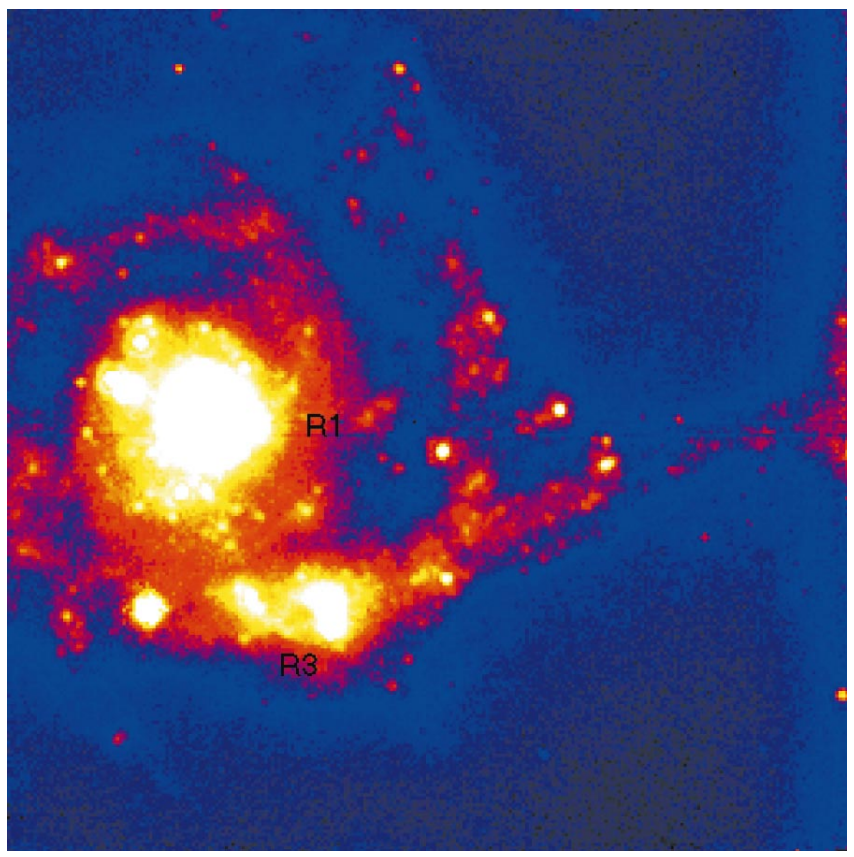

FIG. $1 g$

has strong $[\mathrm{O} \mathrm{III}]$ and blue emission, and these properties are also confirmed by the spectroscopic study $(\S 3.3$ and Fig. $3 b$ ).

Using NTT and HST data we study and compare the structure of the main optical nuclear knots and giant $\mathrm{H}$ II complexes. The NTT $\mathrm{H} \alpha$ and [O III] images show that R1 and R2 have similar morphology: a compact object $(\sim 0$ " 9 FWHM) surrounded by an extended envelope $\left(r \sim 3^{\prime \prime}\right)$. The HST WFPC2 images show new and more detailed structures, especially inside the two optical NTT compact knots. In particular, for R1, Figures $1 d$ and $1 h$ show (1) the nuclear envelope has a spiral disk morphology of $r \sim 3^{\prime \prime} \sim 500 \mathrm{pc}$ of radius, at face-on position; and (2) even the NTT compact

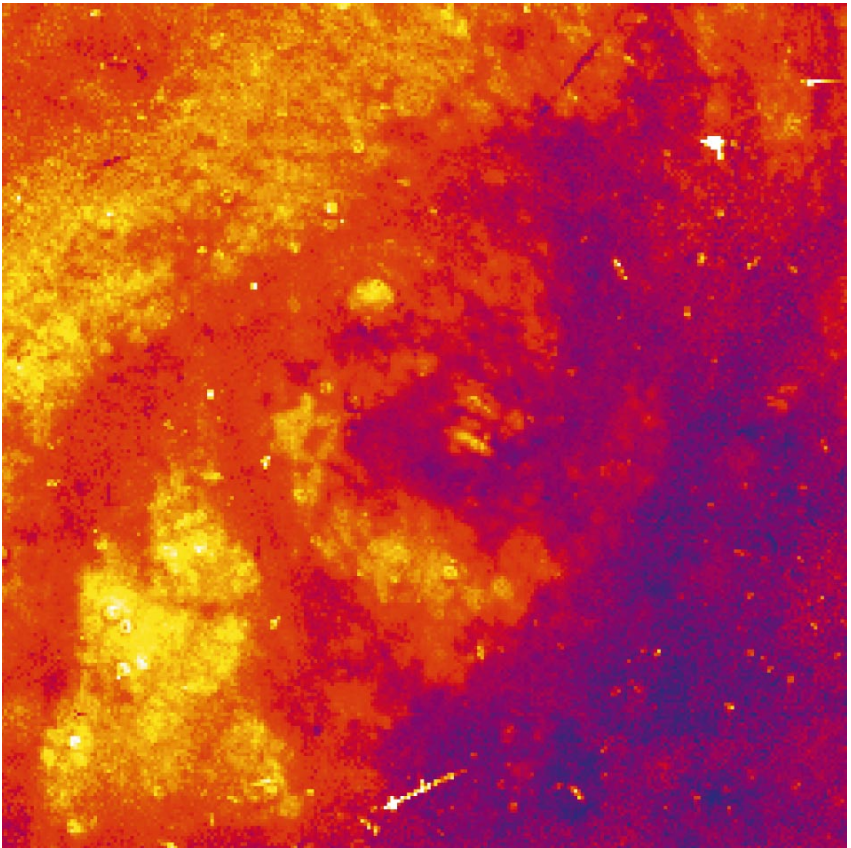

FIG. $1 f$

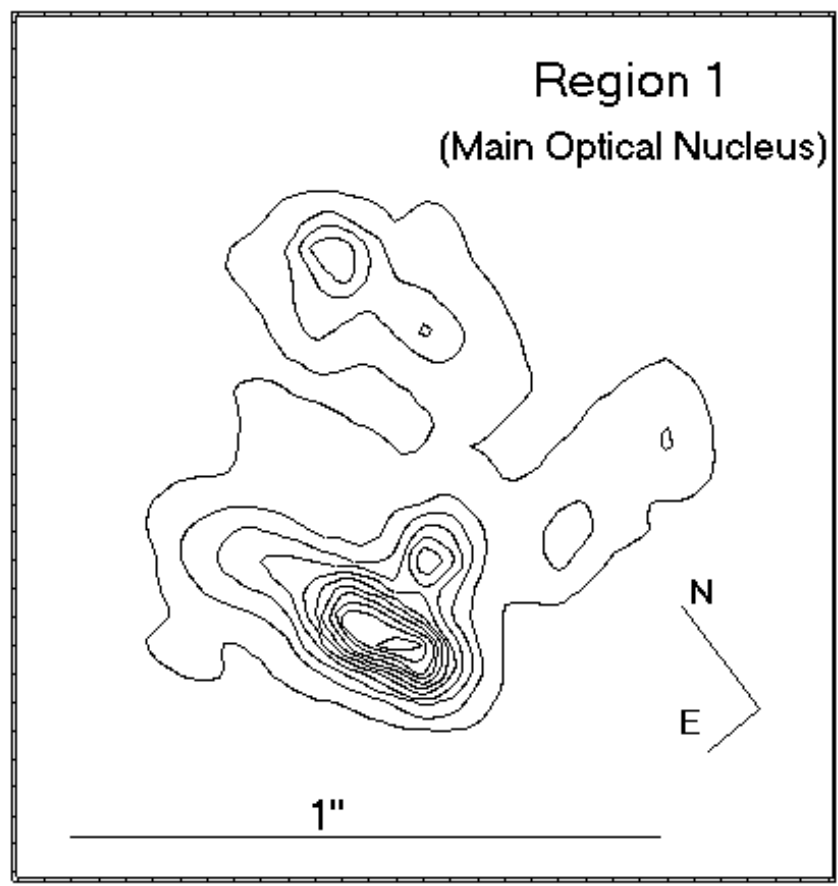

FIG. $1 h$

main nuclear object is an extended structure also with a spiral disk morphology, inside a radius of $\sim 0$ 0.9 (Fig. 1h), plus two small central knots. These two internal spiral disks are a continuation of the external spiral arm I (this is evident in the continuum and color images of the HST data: Figs. $1 e, 1 d, 1 f$, and $1 h$ ). However, there are several obscured areas, probably generated by the presence of dust. The HST observations of R2 (Figs. $1 d$ and 1i) show an envelope with clumpy spiral structure $\left(r \sim 3^{\prime \prime}\right.$; less clear than in $\mathrm{R} 1$, probably due to the high inclination of the spiral disk) and an extended structure plus two small central knots inside a radius of $\sim 0$ ".9 (Fig. $1 i$ ).

Figures $1 h$ and $1 i$ display at subarcsecond scale the struc- 


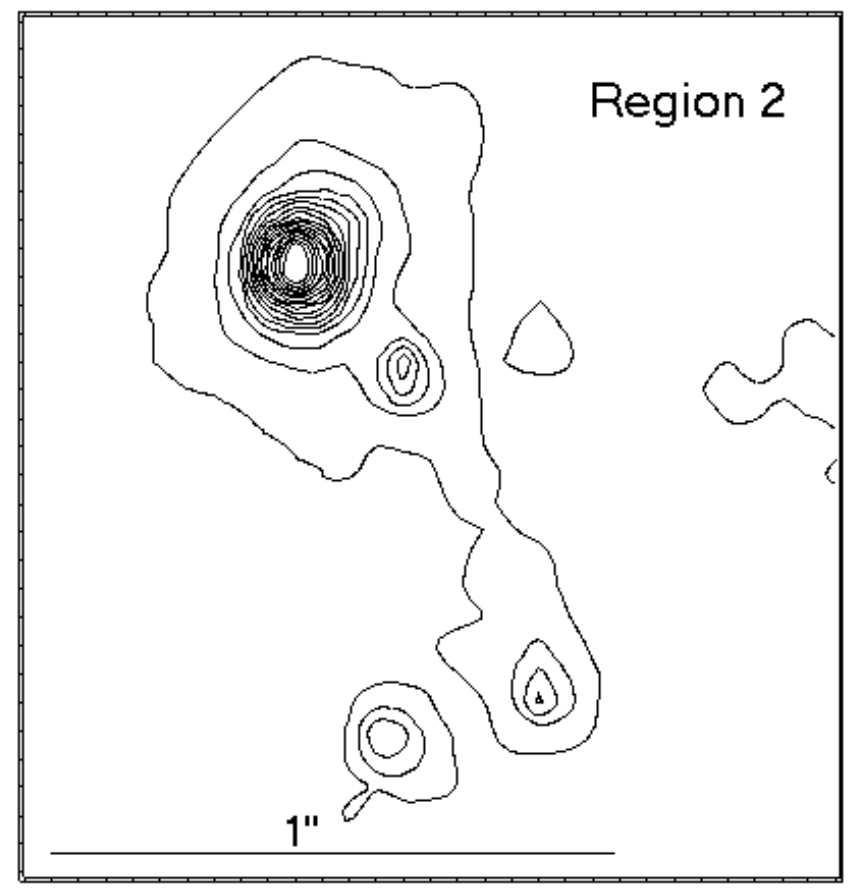

FIG. $1 i$

ture of the two main optical nuclei (R1 and R2). The contours show relatively similar morphology for both regions: (1) a main central knot, which is clearly elongated (50 $\mathrm{pc} \times 25 \mathrm{pc})$ for the R1 core and is compact for R2 core $(r \sim 16 \mathrm{pc})$; (2) a small compact knot close to the main central knot in each region $(r \sim 8 \mathrm{pc})$; and (3) extended spiral disk emission, probably the beginning of the asymmetrical spiral arms (more resolved in R1). These core structures show all blue colors in the crude color map of Figure $1 f$ and also the complete R1 and R2 in our BVI photometry (Table 3, obtained from CASLEO calibrated images). In $\S 4.4$ we discuss the properties of the main blue elongated structure, found in the main optical nucleus of NGC 3256, as a probable blue luminous star cluster. Another interesting point to consider is the properties of the small compact knots detected in each optical nucleus: the measured sizes are the same as those proposed for young star clusters (see Lutz 1991) but also for the accretion radius of a low-mass nuclear black hole (of $10^{7} M_{\odot}$; Heller \& Shlosman 1994).

It is important to note that we found two small nuclear spiral disks $(r \sim 500 \mathrm{pc})$, around regions 1 and 2 (the main optical knots/nuclei), which were detected in the continuum and $\mathrm{H} \alpha$ ionized gas emission (using HST WFPC2 and ESO NTT-SUSI images). The morphology suggests that these spiral disks are probably counterrotating. Sakamoto et al. (1999) found in Arp 220 two similar nuclear/small conterrotating disks $(r \sim 100 \mathrm{pc})$ of molecular gas around the two optical nuclei with masses of $\sim 2.0 \times 10^{9} M_{\odot}$.

The optical obscured R3 shows for wavelength $\lambda \geq 3.75$ $\mu \mathrm{m}\left(\sim L^{\prime}\right.$ filter) typical properties of a nuclear $\mathrm{H}$ II region; i.e., compact and strong continuum emission, typical multiwavelength color index, etc. (Kotilainen et al. 1995; Zenner \& Lenzen 1993; Moorwood \& Oliva 1994; Norris \& Forbes 1995). The HST NICMOS images (which reach only $\lambda \leq 2.5 \mu \mathrm{m}$ ) show that R3 has weak near-IR emission, and it appears to be connected to arm III (Fig. 1g). In general, these short-exposure HST NICMOS images show the strongest circumnuclear knots, and Figure $1 g$ displays mainly regions 1 and 3 and part of the arms $\left(19^{\prime \prime} \times 19^{\prime \prime}\right.$ NIC2 field). In particular, these images show that arm III is also connected to or emanates from R3 (see Fig. 1g).

We note here some general photometric (Table 3) properties of the circumnuclear regions: (1) regions $4,6,8$, and 9 are the strong blue knots in arm II (close to R2); (2) R5 is a strong $\mathrm{H} \alpha$ knot at the end of arm I (region " $\mathrm{X}$ " in Feast \& Robertson 1978); (3) R7a and R7b are the two main $\mathrm{H} \alpha$ knots in arm III (region " $S$ " in Feast \& Robertson 1978 and "A" in Laubert et al. 1978). The R10 (7".3 north and 5".0 west, from the main optical nucleus) is a strong $\mathrm{H} \alpha$ knot in arm I, and it is close to the third illumination source of polarimetry (at 6"1 north and 3".5 west), detected by Scarrott et al. (1996). The spectra and physical conditions of some of these regions are presented and analyzed in $\S \S 3.3$ and 4.4 .

Other, interesting central regions (Table 3) are as follows: (1) R11, R13, R14, and R15 are bright knots inside arm I (in the north area); (2) R12 is a very blue knot inside a secondary arm, part of the main arm I; (3) R16 is a strong $\mathrm{H} \alpha \mathrm{knot}$, located in a secondary or probably obscured fourth arm; (4) R17 is a knot in the disrupted southeast external arm; (5) $\mathrm{R} 18$ is an $\mathrm{H} \alpha$ " isolated " knot, located to the north of $\mathrm{R} 7 \mathrm{~b}$.

It is interesting to note (Table 3 ) that there is an evident continuity in the photometric properties of each spiral arm and also with each corresponding nucleus. In particular, in arm I there is continuity in colors between regions $1,10,11$, $12,13,14,15$, and 4 . In arm II, continuity is detected between regions $2,4,8,9$, and 6 , and in arm III, continuity appears between regions $3,7 \mathrm{a}$, and $7 \mathrm{~b}$. On the other hand, the strong blue colors found in arm II and R2 could be related to intrinsic properties plus the fact that this is probably the nearest arm (Figs. $1 c$ and $1 d$ ).

The [O III] $\lambda \lambda 4959-5007$ narrowband image shows - in general - a similar morphology to the $\mathrm{H} \alpha$ image (Fig. 1c): 
TABLE 3

OpticAl AND IR PhOtometric DATA of NGC 3256

\begin{tabular}{|c|c|c|c|c|c|c|c|c|}
\hline Regions and [Offset $\left.{ }^{\mathrm{a}}(\operatorname{arcsec})\right]$ & $B-V$ & $V-I$ & $V$ & $U-B$ & $I-K$ & $H-K$ & $K^{\prime}-L^{\prime}$ & $A_{V}^{\mathrm{b}}$ \\
\hline $\mathrm{R} 1[00-00-] \ldots \ldots \ldots \ldots \ldots$ & 0.93 & 1.55 & 14.13 & $\ldots$ & 3.09 & 0.48 & 0.95 & $\begin{array}{c}\geq 2.4 \\
\geq[7.8]\end{array}$ \\
\hline R2 [01N05E $] \ldots \ldots \ldots \ldots \ldots \ldots$ & 0.66 & 1.26 & 14.28 & $\cdots$ & 3.26 & 0.61 & $\cdots$ & $\geq 1.7$ \\
\hline R3 [05S00-] ..................... & 1.98 & 2.10 & 16.37 & $\ldots$ & 2.01 & 0.20 & 1.93 & $\begin{array}{c}\geq 5.3 \\
\geq[10.7]\end{array}$ \\
\hline R4 [03N08E] .................... & 0.63 & 1.24 & 14.51 & $\ldots$ & $\ldots$ & $\ldots$ & $\ldots$ & $\geq 1.7$ \\
\hline R5 [01N17E] ...................... & 0.71 & 1.31 & 16.42 & $\ldots$ & $\ldots$ & $\ldots$ & $\ldots$ & $\geq 2.0$ \\
\hline R6 [03N06W] $\ldots \ldots \ldots \ldots \ldots \ldots$ & 0.94 & 1.70 & 15.37 & $\ldots$ & $\ldots$ & $\ldots$ & $\ldots$ & $\geq 2.3$ \\
\hline $\mathrm{R} 7 \mathrm{a}[07 \mathrm{~S} 12 \mathrm{~W}] \ldots \ldots \ldots \ldots \ldots \ldots$ & 0.98 & 1.64 & 15.51 & 0.38 & $\ldots$ & $\ldots$ & $\ldots$ & $\geq 3.0$ \\
\hline $\mathrm{R} 7 \mathrm{~b}[05 \mathrm{~S} 19 \mathrm{~W}] \ldots \ldots \ldots \ldots \ldots \ldots$ & 1.14 & 0.91 & 15.51 & $\ldots$ & $\ldots$ & $\ldots$ & $\ldots$ & $\geq 3.0$ \\
\hline R8 [04N04E] $\ldots \ldots \ldots \ldots \ldots \ldots$ & 0.64 & 1.27 & 14.57 & $\cdots$ & $\cdots$ & $\cdots$ & $\cdots$ & $\ldots$ \\
\hline 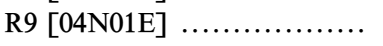 & 0.77 & 1.34 & 14.43 & $\ldots$ & $\ldots$ & $\ldots$ & $\ldots$ & $\ldots$ \\
\hline $\mathrm{R} 10[07 \mathrm{~N} 05 \mathrm{~W}] \ldots \ldots \ldots \ldots \ldots$ & 0.83 & 1.33 & 15.03 & $\ldots$ & $\ldots$ & $\ldots$ & $\ldots$ & $\ldots$ \\
\hline R11 [10N03W] $\ldots \ldots \ldots \ldots \ldots \ldots$ & 0.76 & 1.16 & 15.45 & $\cdots$ & $\cdots$ & $\cdots$ & $\ldots$ & $\ldots$ \\
\hline $\mathrm{R} 12$ [16N01E] $\ldots \ldots \ldots \ldots \ldots \ldots$ & 0.77 & 1.33 & 16.37 & $\ldots$ & $\cdots$ & $\ldots$ & $\ldots$ & $\ldots$ \\
\hline R13 [12N00- $\ldots \ldots \ldots \ldots \ldots \ldots$ & 0.59 & 1.41 & 15.86 & $\ldots$ & $\ldots$ & $\ldots$ & $\ldots$ & $\ldots$ \\
\hline 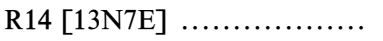 & 0.84 & 1.33 & 16.28 & $\ldots$ & $\ldots$ & $\ldots$ & $\ldots$ & $\ldots$ \\
\hline 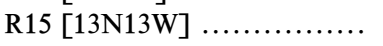 & 0.74 & 1.36 & 16.45 & $\ldots$ & $\ldots$ & $\ldots$ & $\ldots$ & $\ldots$ \\
\hline $\mathrm{R} 16[06 \mathrm{~S} 06 \mathrm{E}] \ldots \ldots \ldots \ldots \ldots \ldots$ & 0.58 & 1.86 & 14.96 & $\ldots$ & $\ldots$ & $\ldots$ & $\ldots$ & $\ldots$ \\
\hline R17 [13S10E $] \ldots \ldots \ldots \ldots \ldots \ldots$ & 0.85 & 1.48 & 17.26 & $\ldots$ & $\ldots$ & $\ldots$ & $\ldots$ & $\ldots$ \\
\hline $\mathrm{R} 18[12 \mathrm{~N} 14 \mathrm{~W}] \ldots \ldots \ldots \ldots \ldots$ & 0.98 & 1.34 & 16.97 & $\ldots$ & $\ldots$ & $\ldots$ & $\ldots$ & $\ldots$ \\
\hline 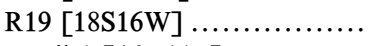 & 0.69 & 1.28 & 17.26 & $\ldots$ & $\ldots$ & $\ldots$ & $\ldots$ & $\ldots$ \\
\hline E-tail $1[46 \mathrm{~N} 11 \mathrm{E}] \ldots \ldots \ldots \ldots$ & 0.86 & 1.44 & 18.81 & $\ldots$ & $\ldots$ & $\ldots$ & $\ldots$ & $\ldots$ \\
\hline E-tail $2[55 \mathrm{~N} 23 \mathrm{E}] \ldots \ldots \ldots \ldots$ & 0.71 & 1.23 & 18.77 & $\ldots$ & $\ldots$ & $\ldots$ & $\ldots$ & $\ldots$ \\
\hline E-tail $3[57 \mathrm{~N} 65 \mathrm{E}] \ldots \ldots \ldots \ldots . . .$. & 0.88 & 1.35 & 19.04 & $\ldots$ & $\ldots$ & $\ldots$ & $\ldots$ & $\ldots$ \\
\hline E-tail $4[48 \mathrm{~N} 111 \mathrm{E}] \ldots \ldots \ldots \ldots$ & 0.85 & 1.11 & 19.51 & $\ldots$ & $\ldots$ & $\ldots$ & $\ldots$ & $\ldots$ \\
\hline W-tail $1[09 \mathrm{~N} 83 \mathrm{~W}] \ldots \ldots \ldots . .$. & 0.58 & 0.98 & 19.96 & $\ldots$ & $\ldots$ & $\ldots$ & $\ldots$ & $\ldots$ \\
\hline W-tail $2[71 \mathrm{~N} 159 \mathrm{~W}] \ldots \ldots \ldots$ & 0.51 & 1.21 & 19.98 & $\ldots$ & $\ldots$ & $\ldots$ & $\ldots$ & $\ldots$ \\
\hline W-tail $3[73 \mathrm{~N} 159 \mathrm{~W}] \ldots \ldots \ldots$ & 0.62 & 1.01 & 19.85 & $\ldots$ & $\ldots$ & $\ldots$ & $\ldots$ & $\ldots$ \\
\hline W-tail $4[129 \mathrm{~N} 198 \mathrm{~W}] \ldots \ldots \ldots$ & 0.64 & 0.35 & 20.39 & $\ldots$ & $\ldots$ & $\ldots$ & $\ldots$ & $\ldots$ \\
\hline SW Loop $[71 \mathrm{~S} 41 \mathrm{~W}] \ldots \ldots \ldots$ & 1.05 & 1.59 & 20.03 & $\ldots$ & $\ldots$ & $\ldots$ & $\ldots$ & $\ldots$ \\
\hline
\end{tabular}

Note. $-B-V, V-I$, and $V$ are from this work (aperture radii of $\sim 3^{\prime \prime}$ ); and $U-V, I-K, K^{\prime}-L^{\prime}, A_{V}$ from Kotilainen et al. 1995 and Zenner \& Lenzen 1993.

${ }^{\text {a }}$ Offset from northern nucleus.

b Values of internal absorption obtained from optical lines emission (from Table 4) and from IR lines/continuum emission (from the work of Kotilainen et al. 1995; values in square brackets). These are lower limits according to the ISO results (Rigopoulou et al. 1996).

extended triple asymmetrical spiral arm structure emanating from three different nuclei. However, there are some differences; in particular, arm II and R2 show strong [O III] emission.

The 3 and $6 \mathrm{~cm}$ radio observations of the central region of this merger (Norris \& Forbes 1995) show extended continuum emission with two strong compact peaks at the position of R1 and R3 plus three weaker/secondary peaks at the position of regions 2,7 , and 10 . They associated the two main compact radio continuum peaks to nonthermal emission from supernovae remnants, which would occur at a rate of one every $3 \mathrm{yr}$ (and they suggested that the two main compact knots are the original nuclei of the two galaxies that collide).

Finally, we note that the continuum emission $(B, V, I, J$, $H, K^{\prime}, L^{\prime}, 3-6 \mathrm{~cm}$ ) and the emission lines ([O III] $\lambda \lambda 4959+5007, \mathrm{H} \alpha+[\mathrm{N}$ II $] \lambda \lambda 6548+6584, \mathrm{Br} \gamma,[\mathrm{Fe}$ II $], \mathrm{CO}$ (2-1)) show extended and similar properties, in a radius of 5-6 kpc (Figs. 1b,1c,1d,1e, and 1g; and also Norris \& Forbes 1995; Kotilainen et al. 1995; Aalto et al. 1991; Moorwood \& Oliva 1994). This suggests a starburst as the same, dominant energy source at optical, IR, millimeter, and centimeter wavelengths. On the other hand, these facts are in good agreement with the suggestion that NGC 3256 is a relatively advanced merger, which is still far from the state of fusion of the original nuclei, and has a high concentration of ionized and molecular gas in the nuclear region. Therefore, interesting processes could be still observed in this merger; for example, the inflow of the ionized $\mathrm{H} \alpha$ gas.

\subsubsection{The Outer Regions $\left(r \geq 30^{\prime \prime} \sim 5 \mathrm{kpc}\right)$}

The external extended envelope $(r \sim 9 \mathrm{kpc})$ shows a rhomboidal shape (Figs. $1 a$ and $1 b$ ) and includes - in the southern part - two probable disrupted arms. We found, for $r \geq 30$ ", "radial filaments" and dust lines emerging from the central region (see Fig. 1b). In this area Scarrott et al. (1996) measured very extended polarization $(r \sim 7-9 \mathrm{kpc})$ attributed to dust reflection and galactic wind. This is consistent with our detection of outflow in the nuclear starburst and extended emission regions (see $\S 3.2 .2$ ). Similar external dusty filaments were found in nearby starburst galaxies with galactic wind (e.g., M82, NGC 253, NGC 1808, and others; see, for references, Franco \& Ferrara 1992).

On the other hand, in the southern part of the external regions, there are only a few strong emitting structures (Fig. $1 b$ ). This fact could be explained by the presence of strong reddening in these areas (Scarrott et al. 1996).

In the outer part of the main body of this merger, there are two disrupted externals arms, which are probably connected to external loops (Figs. $1 b$ and $1 a$ ). In particular, the 
southwest external arm shows a more extended morphology and a clear connection to the southeast loop (Fig. 1b). Additionally, R17 (which is inside of the southeast disrupted external arm) has photometric continuity with the southwest loop (Table 3).

Table 3 also includes the $B V I$ photometric properties of several interesting external regions: (1) region E-tail/1, Etail/2, E-tail/3, and E-tail/ 4 are the four main/strong blue knots inside the $\mathrm{E}$ tidal tail; and (2) region W-tail/1, W-tail/ $2, \mathrm{~W}$-tail/3, and $\mathrm{W}$-tail/4 are the four main blue knots inside the $\mathrm{W}$ tail.

Several condensations show blue colors, and the more intense and bluer regions are located at the west tail (see Table 3). These blue regions are probably associated with star formation processes, as has been observed in similar major mergers IRAS 19254-7245, NGC 4038/39, and Mrk 231 (by Mirabel, Lutz, \& Maza 1991; Colina et al. 1991; Mirabel, Dottori, \& Lutz 1993; Lípari et al. 1994).

\subsection{The Ionized Gas Kinematics}

NGC 3256 is probably the nearest "advanced" major merger known; however, the main kinematical properties of this system remain undefined. Furthermore, different works (e.g., Feast \& Robertson 1978; Sargent et al. 1989; Scarrott et al. 1996) suggested that a detailed kinematics study of this merger is required.

Our kinematic observations consist of more than 1000 individual spectra, which were extracted from long-slit frames obtained at $\sim 25$ position angles (with an instrumental width of $\sim 90 \mathrm{~km} \mathrm{~s}^{-1} \mathrm{FWHM}$, in the sky emission lines). We measured the velocities from the centroids of the stronger emission lines $\mathrm{H} \alpha$, [N II] $\lambda 6584$, and [S II] $\lambda \lambda 6717-6731$, fitting Gaussians. The long-slit positions were obtained mainly passing through the main optical nucleus (each $10^{\circ}-$ $20^{\circ}$ ) in order to use the nucleus velocity as reference, i.e., as the zero value in the velocity field (VF). Therefore, each point in the VF is the result of the determination of (1) the velocity obtained from high-quality spectra $(\mathrm{S} / \mathrm{N} \sim 10-30)$ and (2) the "relative" position, which is accurate on the scale of both VFs $(0.5$ and $1 ")$ presented here.

An important point is the analysis of multiple components in each emission line $(\mathrm{H} \alpha,[\mathrm{N}$ II ] $\lambda 6584$, [S II] $\lambda \lambda 6717-6731$ ). For Cen A and NGC 1052, 4550, and 7332, this type of study was made by Bland, Taylor, \& Atherton (1987), Rubin, Graham, \& Kenney (1992), and Plana \& Boulesteix (1996). In NGC 3256, first we study only the main emission-line component, which is very strong in the central region. However, in the outer regions the presence of multiple components - with similar weak strengthrequired a detailed study (\$3.2.3).

The inclination value adopted as a first approximation in our kinematics study was $i=40^{\circ}$ (from Feast \& Robertson 1978), and the final adopted value was $i=42^{\circ} \pm 5^{\circ}$, which minimized the sum of the squares of the residual velocity values (in the regions where circular motion prevails). Additionally, we assume that the nearest side of the merger is the north part, i.e., the less obscured area.

\subsubsection{The Kinematics in the Nuclear and Central Regions $\left(r \leq 30^{\prime \prime} \sim 5 \mathrm{kpc}\right)$}

Figures $2 a$ and $2 b$ show the $\mathrm{H} \alpha$ velocity field - color-map and contours-for the central 40" $\times 40^{\prime \prime}(\sim 7 \mathrm{kpc} \times 7 \mathrm{kpc}$, and $r_{\max } \sim 30^{\prime \prime} \sim 5 \mathrm{kpc}$ ), with a spatial sampling of $00^{\prime \prime} 76$ arranged in a grid of $1^{\prime \prime} \times 1^{\prime \prime}$ boxes. The errors/ uncertainties vary from approximately $\pm 15 \mathrm{~km} \mathrm{~s}^{-1}$ in the nuclear and central regions up to $\sim 30 \mathrm{~km} \mathrm{~s}^{-1}$ for the weakest lines away from the nuclear areas. Approximately $35 \%$ of the grid points of the velocity field were obtained by linear interpolation of observed radial velocity, mainly in the outer radii (the central regions are oversampled).

We observed in the nuclear region of the VF $\left(r \leq 5^{\prime \prime} \sim 1\right.$ $\mathrm{kpc}$ ) the typical shape of circular motion, i.e., the "spider" shape (Fig. 2b). Additionally, we determine the precise location of the kinematical center of rotation using different methods (interception of isovelocity lines, square root of nuclear rotation curve residuals, etc.). This center is located $3^{\prime \prime}$ south and $1^{\prime \prime}-15^{\prime \prime}$ west from the main optical nucleus, very close to the line that connects the main optical nucleus and the obscured nuclear knot (R3).

The velocity pattern in the neighborhood of the kinematical center and the main optical nucleus $\left(r \leq 4^{\prime \prime} \sim 1\right.$ $\mathrm{kpc}$ ) was studied using the plot $V R$ versus cos (P.A. - P.A. 0 ), where P.A. is the position angle of the radial velocity point and P.A..$_{0}$ is the position angle of the kinematical line of nodes. We found rotational pattern around both areas.

The extended $\mathrm{H} \alpha$ color and isovelocity maps (Figs. $2 a$ and $2 b$ ) have an amplitude of $\sim 250 \mathrm{~km} \mathrm{~s}^{-1}$ and show interesting and complex structures: (1) in the negative velocity region (blueshifted) there is a defined lobe, close to arm III (southwest region, with a strong blue peak of $\Delta V \sim-150 \mathrm{~km} \mathrm{~s}^{-1}$ ), showing continuity to the external region and especially to the west tail; (2) another negative lobe close to the third source of polarization (Scarrott et al. 1996; in the northwest area); (3) in the positive velocity region there are three distinct lobes (but they can not be easily associated with any particular structure). There is also continuity of weak positive values, in the north part of the VF, in the direction to the northeast tail.

The systemic velocity $\left(V_{\text {syst }}\right)$ was determined using different methods. First, for each slit position passing through the main optical nucleus, the $V_{\text {syst }}$ was determined as the mean value of the external positions of the corresponding velocity curve. In this way the resulting mean $V_{\text {syst }}$-for all the P.A.'s and corrected by heliocentric motion-was $2815 \pm 7$ $\mathrm{km} \mathrm{s}^{-1}$. In addition, we measure the velocity of the spider center (using the mean velocity of $V_{R 1}=2823 \pm 12 \mathrm{~km} \mathrm{~s}^{-1}$ ) and it is $V_{\text {syst }}=2819 \pm 8 \mathrm{~km} \mathrm{~s}^{-1}$. Therefore, the mean value for the velocity of the system is $V_{\text {syst }}=2817 \pm 10 \mathrm{~km} \mathrm{~s}^{-1}$.

On the other hand, the velocity obtained for the $\mathrm{CO}$ kinematical center was $2800 \pm 30 \mathrm{~km} \mathrm{~s}^{-1}$ (Aalto et al. 1991; and § 4.3). Previously, Feast \& Robertson (1978) found for the system and $\mathrm{R} 7 \mathrm{a}$ velocities of $2823 \pm 25 \mathrm{~km} \mathrm{~s}^{-1}$ and $2710 \pm 25 \mathrm{~km} \mathrm{~s}^{-1}$, respectively. Zenner \& Lenzen (1993) obtained for the main optical nucleus a value of $2818 \pm 18$ $\mathrm{km} \mathrm{s}^{-1}$. Therefore, the velocity values obtained in this work are in good agreement with those reported previously.

The three noncoplanar arms $(\S 3.1)$ can be traced through the residual lobes of the VF, and one of them (arm II) has clearly an opposite sense of rotation, in agreement with the general $\mathrm{H} \alpha$ and broadband morphology (Figs. $1 c$ and $1 d$ ).

Three superposed rotation curves (RCs) were found from the extraction in the VF of a wide-aperture angular sector $\left(\theta \sim 80^{\circ}\right)$ at P.A. $\sim 90^{\circ}$. Then, we separated these RCs using extraction of small-aperture angular sector $\theta$ of $30^{\circ}$, around P.A. $\sim 55^{\circ}, \sim 90^{\circ}$, and $\sim 130^{\circ}$ (Fig. $2 c$ ). These RCs could be associated with the three main lobes observed in the posi- 

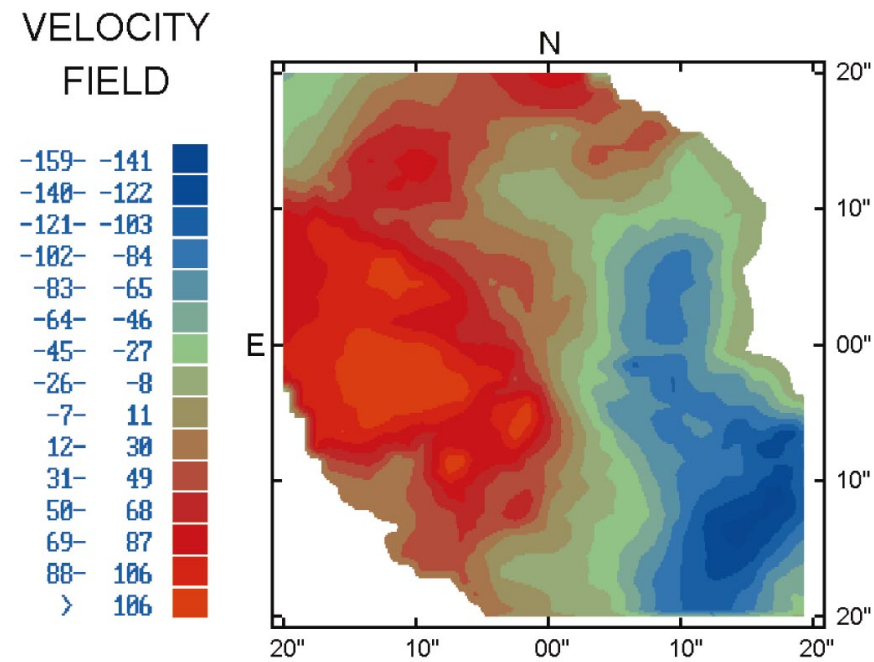

FIG. $2 a$

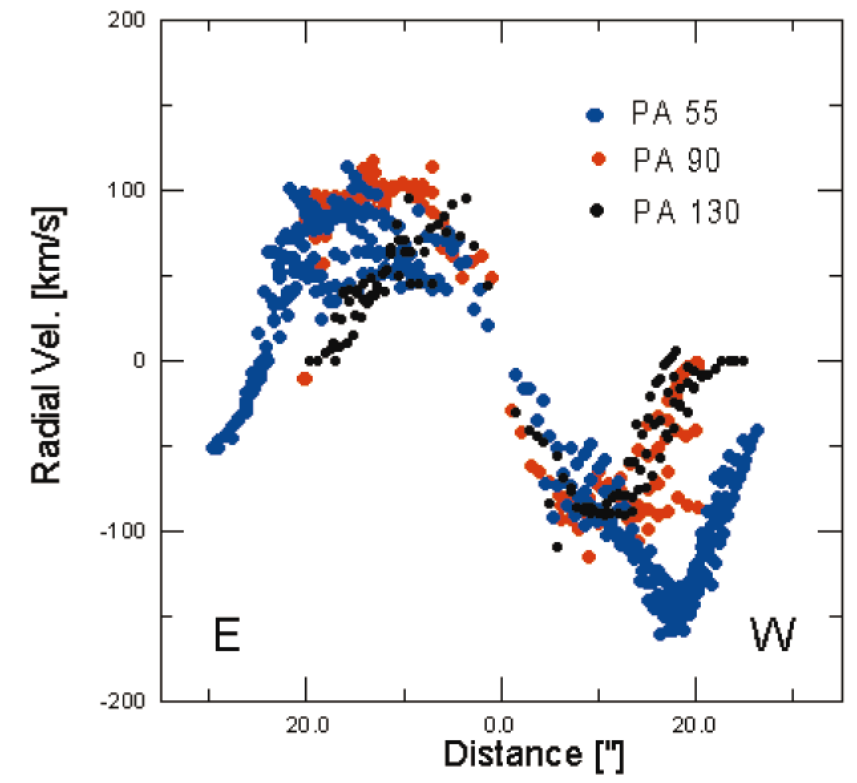

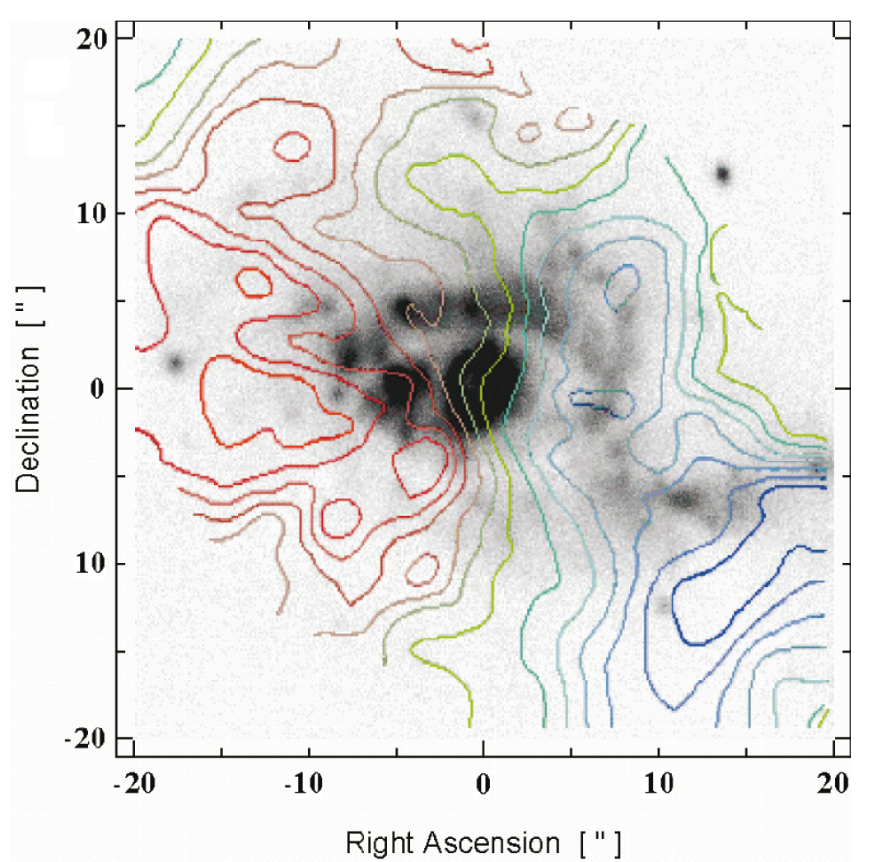

FIG. $2 b$

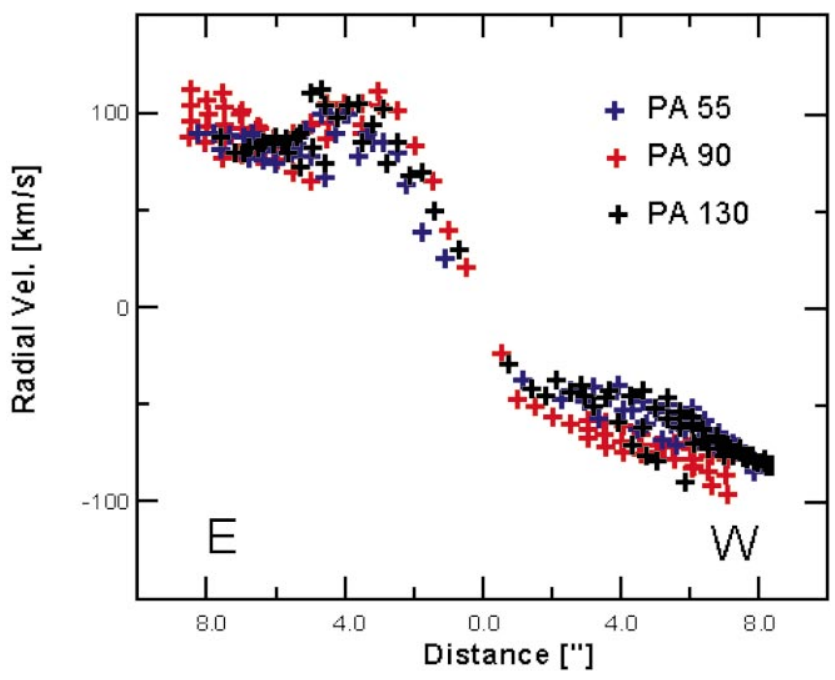

FIG. $2 d$

FIG. $2 c$

FIG. 2.-(a) Color map of the H $\alpha$ velocity field, for the central region $\left(40^{\prime \prime} \times 40^{\prime \prime} \sim 7 \mathrm{kpc} \times 7 \mathrm{kpc}\right.$; with a spatial resolution of $1^{\prime \prime} \times 1^{\prime \prime}$, and errors of \pm 15 $\mathrm{km} \mathrm{s}^{-1}$ ). (b) Contours of $\mathrm{H} \alpha$ velocity field superposed to a $\mathrm{H} \alpha+[\mathrm{N} \mathrm{II}]$ NTT image, for the central region (with similar resolution and error to [a]). The values of the lowest contour and interval are $-120 \mathrm{~km} \mathrm{~s}^{-1}$ and $+20 \mathrm{~km} \mathrm{~s}^{-1}$. (c) Three rotation curves found in the central region of NGC 3256, at P.A. 55 , $90^{\circ}$, and $130^{\circ}$, with spatial resolution of $1^{\prime \prime} 0$, obtained first from a wide opening angle $\theta=80$ and then for $\theta=30$ (see the text). (d) Three rotation curves obtained from the nuclear region of NGC 3256, at P.A. $55^{\circ}, 90^{\circ}$, and $130^{\circ}$, with spatial resolution of 0.5 , and obtained from an opening angle $\theta=20$; showing a superposed inflow shape (see the text). (e) Contour of $\mathrm{H} \alpha+[\mathrm{N} \mathrm{II}] \lambda \lambda 6548,6583$ region of a spectra along P.A. $110^{\circ}$ through the main optical nucleus, showing clearly the outflow/blue nuclear component (note that this blue component shows stronger [N II] $\lambda 6583$ emission than $\mathrm{H} \alpha$ ). $(f)$ Outflow and radial velocity vs. axial distance (from the main optical nucleus) at P.A. $18^{\circ}$.

tive and negative regions of the $\mathrm{VF}$ and show the typical "sinusoidal shape" of merger galaxies (Schweizer 1982; Rubin \& Ford 1983; Zepf 1993). In $\S 4.3$ we discuss the properties of these RCs.

Moorwood \& Oliva (1994), from a Br $\gamma$ velocity map, already suggested the presence of more than one kinematical axis in NGC 3256. They also proposed a major kinematical axis at P.A. $\sim 65^{\circ}$ (which is close to the P.A. $\sim 55^{\circ}$ of one of our RCs). Previously, Feast \&
Robertson (1978) and Aalto et al. (1991) suggested a major kinematical axis at P.A. $\sim 110^{\circ}$ and $\sim 90^{\circ}$, respectively.

The nuclear VF $\left(r \leq 5^{\prime \prime}-7^{\prime \prime} \sim 1 \mathrm{kpc}\right.$, with 0 ."5 sampling) showed stronger evidence for the presence of three RCs (P.A. $\sim 55^{\circ}, \sim 90^{\circ}$, and $\sim 130^{\circ}$; Fig. $2 d$ ) superposed on the generally smooth VF. The center for these RCs is located very close to the main nucleus. These sinusoidal curves have a symmetric "break" at $\sim \pm 4$ " $(r \sim 700 \mathrm{pc})$, and we also obtained a similar result using the extended VF with 1 " 


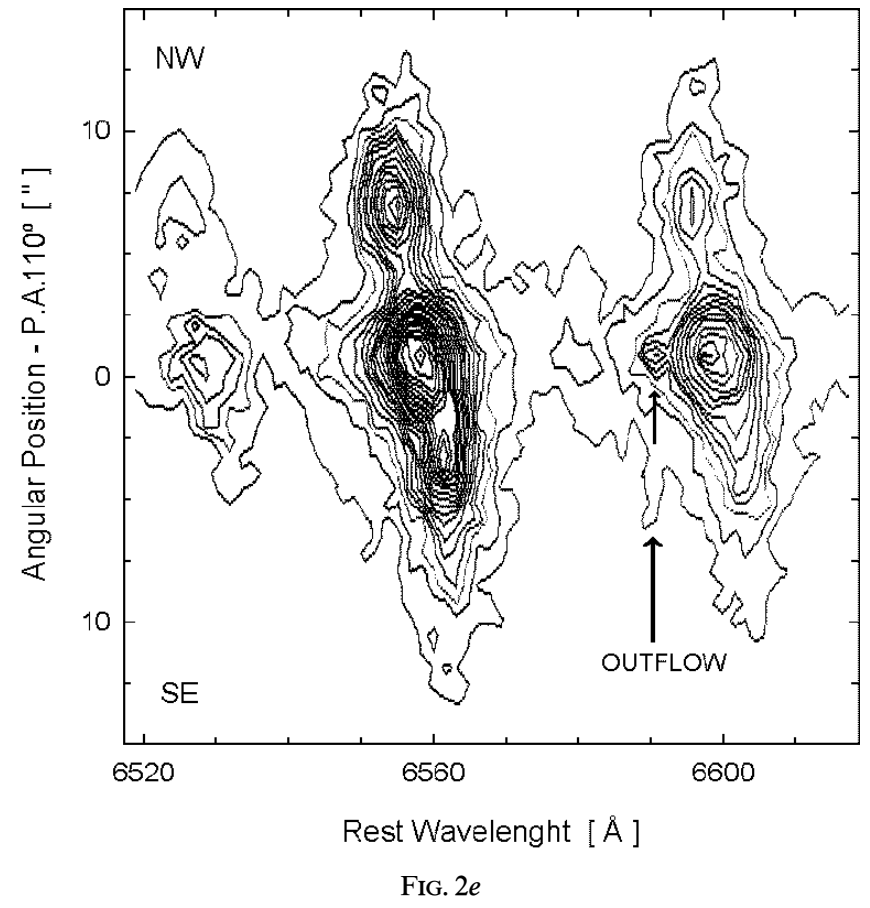

resolution (but less clearly). The superposed sinusoidal shape appears in the nuclear VF in the P.A. range of $40^{\circ}-$ $130^{\circ}$. Additionally, this shape in the RCs is very similar to that found in the nuclear velocity curve of the Seyfert 1 galaxy NGC 6860. This type of superposed curve could be associated with the presence of inflow (Lípari, Tsvetanov, \& Macchetto 1993b), since the increase in the nuclear rotation curve - in relation to the general $\mathrm{RC}$ - is due to the presence of a departure of the general smooth gravitational potential (a major value, in the nuclear region), which could generate the inflow/outflow of the material. We discuss in $\S$ 4.3 these two alternatives.

It is important to note that the region with this probable inflow $\left(r \leq 4^{\prime \prime}\right)$ is approximately where the HST data $(\S 3.1)$ show a spiral structure around the main optical nucleus.

\subsubsection{The Kinematics of the Outflow}

We found direct evidence of outflow in the main optical nucleus (R1) of NGC 3256: practically all the spectra show - in the nuclear region-blue components in the $\mathrm{H} \alpha$, $[\mathrm{N}$ II] $\lambda 6584$, and [S II] $\lambda 6717$ emission lines (Fig. $2 e$ ), with interesting variations for different P.A.'s. In order to check these variations we observed the same P.A. using different instrumental configurations, and we obtained the same result: there is a blue/outflow component for practically all the P.A.s. However, in the interval of P.A. $45^{\circ}-80^{\circ}$ this component practically disappears or is very weak.

Specifically, for P.A.'s without outflow component (e.g., P.A. $45^{\circ}$ ) we made the test of increasing the long-slit aperture (from $1^{\prime \prime}$ to $3^{\prime \prime}$ ), and in all the cases - for this P.A.- the blue component was not detected. A second test was performed for P.A.'s with outflow component (e.g., PA $110^{\circ}$ ); moving the long-slit position very close to the nucleus, we detected the blue component only when the long-slit was exactly at the center of the nucleus. Therefore, we conclude that the outflow is strong mainly in the nuclear area and without - or with a very weak - a component at P.A.

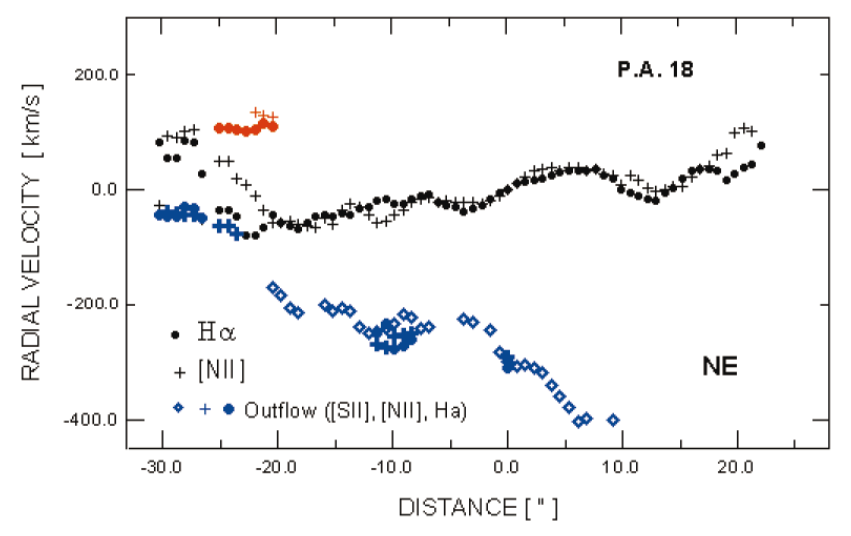

FIG. $2 f$ $45^{\circ}-80^{\circ}$ (i.e., nuclear bipolar outflow, with an opening angle $\left.\theta \sim 140^{\circ}\right)$.

We note that this blue outflow component was clearly detected only using data of relatively high spectral resolution $\left(\sim 90 \mathrm{~km} \mathrm{~s}^{-1} \mathrm{FWHM}\right)$. Additionally, the velocity of this nuclear outflow is $V_{\text {nucl.outflow }} \sim 350 \mathrm{~km} \mathrm{~s}^{-1}$, and the FWHM is $\sim 130 \mathrm{~km} \mathrm{~s}^{-1}$. In $\S 4.5$ this component will be associated to the nuclear starburst.

On the other hand, in order to study the outflow component in the central and external regions, long-exposure spectroscopic observations were performed (co-added spectra of $\sim 3-4 \mathrm{hr}$; see Table 1), in particular, at two selected position angles: P.A. $18^{\circ}$ and $70^{\circ}$ (close to the minor kinematic axis and to the R7, respectively). Figure $2 f$ shows the radial velocity versus axial distance (from the nucleus) for "the main and the outflow components" at PA $18^{\circ}$ and for the $\mathrm{H} \alpha, \mathrm{N}$ [II], and [S II] emission lines. We detected extended outflow-in the emission lines [S II] $\lambda 6717,[\mathrm{~N}$ II $]$ 26584 , and $\mathrm{H} \alpha-$ at different radii, up to $r \sim 30^{\prime \prime}-40^{\prime \prime}$. The outflow component is present in the entire range $\left(r \leq 30^{\prime \prime} \sim 5 \mathrm{kpc}\right.$; Fig. $\left.2 f\right)$ but only in the line [S II] 26717 . This could be explained by a combination of two facts: (1) strong flux in the [S II] outflow component, and (2) the relatively easy detection and measurement of the [S II] $\lambda 6717$ outflow component, since it is located in the blue border of the blend [S II] $\lambda \lambda 6717+6731$ (main component). This extended outflow, reaching $r \sim 5-6 \mathrm{kpc}$, was already suggested by a polarimetry study (Scarrott et al. 1996).

We observed for the galactic wind in NGC 4945 (the nearest starburst and Seyfert nuclei; Lípari et al. 1997) a similar relation in the strength of these three emission lines: strong [N II] and [S II] and weak $\mathrm{H} \alpha$ (see Lípari et al. 1997: their Figs. $3 b$ and $3 c$ ). For NGC 3256, we measure in the extended outflow component the following values of the emission-line ratios: $0.7 \leq\left(\left[\begin{array}{ll}\mathrm{S} & \text { II }\end{array}\right] \lambda \lambda(6717+6731) /\right.$ $\mathrm{H} \alpha) \leq 1.3$ and $0.8 \leq([\mathrm{N} \mathrm{II}] \lambda 6584 / \mathrm{H} \alpha) \leq 1.6$. These values are clearly consistent with shocks driven into clouds accel- 
erated outward by a starburst with galactic wind (see $\S 4.5$; HAM90: their Fig. 14; Lípari et al. 1994, 1997).

3.2.3. The Kinematics in the Outer Regions $\left(r \geq 30^{\prime \prime} \sim 5 \mathrm{kpc}\right)$

$\mathrm{H} \alpha+[\mathrm{N} \mathrm{II}]$ emission is strong only in the nuclear and central area $(r \leq 5 \mathrm{kpc}$; Fig. $1 \mathrm{c})$. In the external areas, the flux and signal-to-noise ratio $(\mathrm{S} / \mathrm{N})$ of the emission lines decrease $(\mathrm{S} / \mathrm{N} \sim 2-5)$, and the errors increase to $\sim 20-40$ $\mathrm{km} \mathrm{s}^{-1}$.

Large-exposure observations show that the $\mathrm{H} \alpha$ ionized gas, in the tails of NGC 3256, presents - in general-a similar velocity behavior to that of the atomic $\mathrm{H}$ I gas in the tails of the merger NGC 7252 (Hibbard et al. 1994): i.e., the external $\mathrm{H} \alpha$ ionized gas is swinging through space in the same sense as the rotation of the central ionized gas.

Specifically, in the western tail the velocity increases from $-100 \mathrm{~km} \mathrm{~s}^{-1}$ at the base of the tail (located at $\sim 22^{\prime \prime}$ west and $16^{\prime \prime}$ south from the R1) to $-130 \mathrm{~km} \mathrm{~s}^{-1}$ at $13^{\prime \prime}-18^{\prime \prime}$ from the base of the tail, and then the velocity gradually decreases. In the eastern tail the kinematical behavior is similar, however, the positive values are smaller in amplitude than those obtained in the western tail.

\subsection{Optical Spectrophotometry}

There are several IR spectophotometric studies of NGC 3256; however, there are only a few general studies at optical wavelengths (see Laubert et al. 1978; HAM90; Aguero \& Lípari 1991). Sargent et al. (1989) already suggested that a detailed spectroscopic study of the "optical knotty" struc- ture of NGC 3256 is required in order to determine clearly the nature of these knots or clumps.

\subsubsection{Optical, UV, and Near-IR Spectrophotometry of Moderate Resolution}

In this section we present results of optical, UV and near-IR spectrophotometry (of moderate spectral resolution: $\sim 290 \mathrm{~km} \mathrm{~s}^{-1}$ FWHM) for the main structures studied in $\S 3.1$, which, combined with the data obtained from photoionization codes (e.g., Ferland 1991) and models of high-metallicity giant $\mathrm{H}$ II regions, massive star formation, and shocks (Garcia Vargas el al. 1997; Pastoriza et al. 1993; Garcia Vargas \& Diaz 1994; Garcia Vargas, Bressan, \& Diaz 1995a, 1995b), will allow us to determine the physical condition in the emitting gas and also in the main ionization sources. These results will be also compared with those obtained previously for the near- and mid-IR wavelengths.

Tables 4 and 5 and Figures $3 a-3 i$ present the results obtained for the main central regions $1,2,4,5,6$, and 7 , plus UV and near-IR spectra of the circumnuclear area [corrected by galactic reddening, with $E(B-V)=0.13$ ] The main conclusions are as follows: (1) all the observed spectra in the central area show similar characteristics, i.e., high-metallicity giant $\mathrm{H}$ in regions or starbursts (extended in a $r \sim 30^{\prime \prime} \sim 5-6 \mathrm{kpc}$; the same area of strong optical, IR, millimeter, and radio continuum and emission lines); (2) the two compact nuclear regions- $\mathrm{R} 1$ and $\mathrm{R} 2-$ show lumi-

TABLE 4

FLUXES OF EMISSION LINES ${ }^{\text {a }}$ OF NGC 3256

\begin{tabular}{|c|c|c|c|c|c|c|c|}
\hline \multirow[b]{2}{*}{$\begin{array}{l}\text { LINES/RATIOS/EW } \\
\left.\text { [Offset }^{c}(\operatorname{arcsec})\right]\end{array}$} & \multicolumn{7}{|c|}{ FLUXES $^{b}$} \\
\hline & $\begin{array}{c}\mathrm{R} 1 \\
{[00-00-]}\end{array}$ & $\begin{array}{c}\mathrm{R} 2+ \\
{[01 \mathrm{~N} 05 \mathrm{E}]}\end{array}$ & $\begin{array}{c}\mathrm{R} 4 \\
{[03 \mathrm{~N} 8 \mathrm{E}]}\end{array}$ & $\begin{array}{c}\mathrm{R} 5 \\
{[02 \mathrm{~N} 18 \mathrm{E}]}\end{array}$ & $\begin{array}{c}\mathrm{R} 6 \\
{[03 \mathrm{~N} 06 \mathrm{~W}]}\end{array}$ & $\begin{array}{c}\mathrm{R} 7 \mathrm{ab} \\
{[07 \mathrm{~S} 12 \mathrm{~W}]}\end{array}$ & $\mathrm{R} 1+\mathrm{R} 2^{\mathrm{d}}$ \\
\hline$[\mathrm{O}$ II $] \lambda 3727 \ldots \ldots \ldots$ & $\ldots$ & $\ldots$ & $\ldots$ & $\ldots$ & $\ldots$ & $\ldots$ & 72.5 \\
\hline $\mathrm{H} \delta \lambda 4101^{\mathrm{e}} \ldots \ldots \ldots \ldots$ & $(4.0)$ & (3.0) & $\ldots$ & $\ldots$ & $\ldots$ & $\ldots$ & 8.6 \\
\hline $\mathrm{H} \gamma \lambda \lambda 340 \ldots \ldots \ldots \ldots$ & 7.0 & 8.3 & 2.2 & 1.4 & 2.5 & $\ldots$ & 14.6 \\
\hline [O III] $\lambda 4363 \ldots \ldots \ldots$ & $\ldots$ & $\ldots$ & $\ldots$ & $\ldots$ & $\ldots$ & $\ldots$ & $(3.0)$ \\
\hline $\mathrm{H} \beta \lambda 48661 \ldots \ldots \ldots \ldots$ & 21.0 & 24.0 & 7.3 & 4.6 & 8.6 & $(11.2)$ & 43.5 \\
\hline$[\mathrm{O}$ III $] \lambda 5007 \ldots \ldots \ldots$ & 7.0 & 10.6 & 3.9 & 2.3 & 4.0 & $(4.8)$ & 19.5 \\
\hline$[\mathrm{Na} \mathrm{I}] \mathrm{D} \lambda 5890 \ldots \ldots$ & $\ldots$ & 3.2 & 1.0 & $\ldots$ & 1.4 & $(0.7)$ & 3.5 \\
\hline$\left[\mathrm{O}_{\mathrm{I}}\right] \lambda 6300 \ldots \ldots \ldots$ & 3.5 & 3.0 & 1.3 & 1.2 & 2.0 & $(4.0)$ & 6.3 \\
\hline $\mathrm{H} \alpha \lambda 6563 \ldots \ldots \ldots \ldots$ & 112.0 & 115.0 & 36.1 & 24.1 & 52.0 & 80.8 & 219.0 \\
\hline$[\mathrm{N}$ II $] \lambda 6583 \ldots \ldots \ldots$ & 65.0 & 47.7 & 13.0 & 9.0 & 21.8 & 34.4 & 85.0 \\
\hline$[\mathrm{S}$ II $] \lambda 6717 \ldots \ldots \ldots$ & 13.0 & 14.3 & 5.4 & 3.0 & 9.1 & 18.8 & 30.0 \\
\hline$[\mathrm{S}$ II $] \lambda 6731 \ldots \ldots \ldots$ & 13.6 & 13.0 & 4.4 & 2.3 & 7.3 & 14.0 & 30.4 \\
\hline$[\mathrm{S}$ III $] \lambda 9069 \ldots \ldots \ldots$ & $\ldots$ & $\ldots$ & $\ldots$ & $\ldots$ & $\ldots$ & $\ldots$ & 23.0 \\
\hline$[\mathrm{S}$ III $] \lambda 9532 \ldots \ldots \ldots$ & $\ldots$ & $\ldots$ & $\ldots$ & $\ldots$ & $\ldots$ & $\ldots$ & 61.0 \\
\hline $\mathrm{H} \alpha / \mathrm{H} \beta \ldots \ldots \ldots \ldots \ldots$ & 5.4 & 4.7 & 5.0 & 5.2 & 6.1 & $(7.2)$ & 5.0 \\
\hline$E(B-V)_{I} \ldots \ldots \ldots \ldots$ & 0.6 & 0.5 & 0.5 & 0.6 & 0.7 & 0.9 & 0.5 \\
\hline \multirow[t]{2}{*}{ Lum $\mathrm{H} \alpha{ }^{\mathrm{f}} \ldots \ldots \ldots \ldots$} & 1.8 & 2.0 & 0.6 & 0.4 & 0.9 & 1.3 & 3.6 \\
\hline & {$[7.3]$} & {$[6.2]$} & [1.9] & {$[1.6]$} & {$[4.3]$} & $\ldots$ & {$[11.4]$} \\
\hline $\mathrm{EW}[\mathrm{O}$ II $](\AA)$ & $\ldots$ & $\ldots$ & $\ldots$ & $\ldots$ & $\ldots$ & $\ldots$ & 44.0 \\
\hline EW H $\beta(\AA) \ldots \ldots \ldots \ldots$ & 23 & 32 & $\dddot{45}$ & 70 & 45 & $(28)$ & 31.5 \\
\hline EW $[\mathrm{O}$ III $](\AA) \ldots \ldots . . . .$. & 8 & 14 & 30 & 38 & 20 & $(7)$ & 17.0 \\
\hline EW $\mathrm{H} \alpha(\AA) \ldots \ldots \ldots \ldots$ & 142 & 235 & 280 & 370 & 210 & 100 & 295.0 \\
\hline $\mathrm{EW}[\mathrm{N} \mathrm{II}](\AA) \ldots \ldots .$. & 77 & 97 & 100 & 145 & 85 & 43 & 135.0 \\
\hline
\end{tabular}

${ }^{\text {a }}$ Observations from CASLEO and CTIO (see the text).

${ }^{b}$ The fluxes are given in units of $10^{-14}$ ergs $\mathrm{cm}^{-2} \mathrm{~s}^{-1}$ and corrected for atmospheric extinction, galactic reddening $[E(B-V)=0.13]$ and redshift. The $\mathrm{R} 2+$ is the $\mathrm{R} 2$ plus the superposed contribution of the border of $\mathrm{R} 4$ (see the text).

${ }^{\mathrm{c}}$ Offset from northern nucleus.

d Observations from CTIO.

e The values between parenthesis are data with low $\mathrm{S} / \mathrm{N}$.

${ }^{\mathrm{f}}$ The luminosities are given in units of $10^{41} \mathrm{ergs} \mathrm{s}^{-1}$. The values in square brackets are data corrected by internal reddening. 


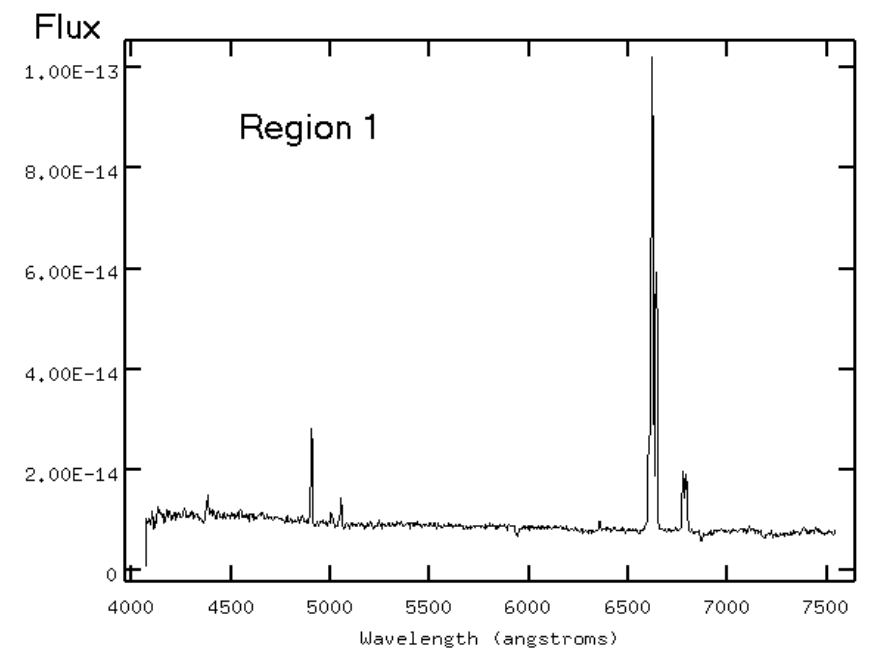

FIG. $3 a$
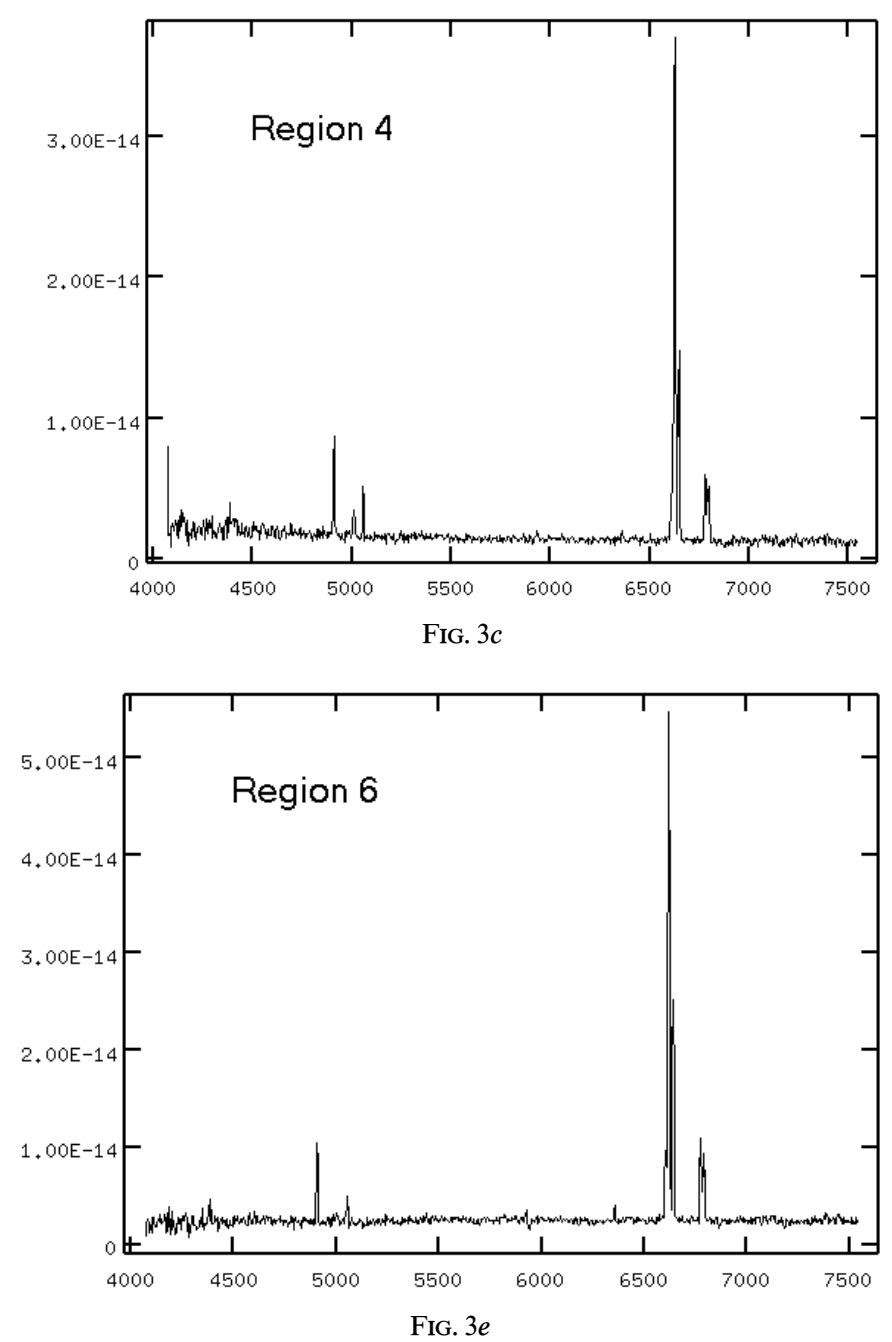
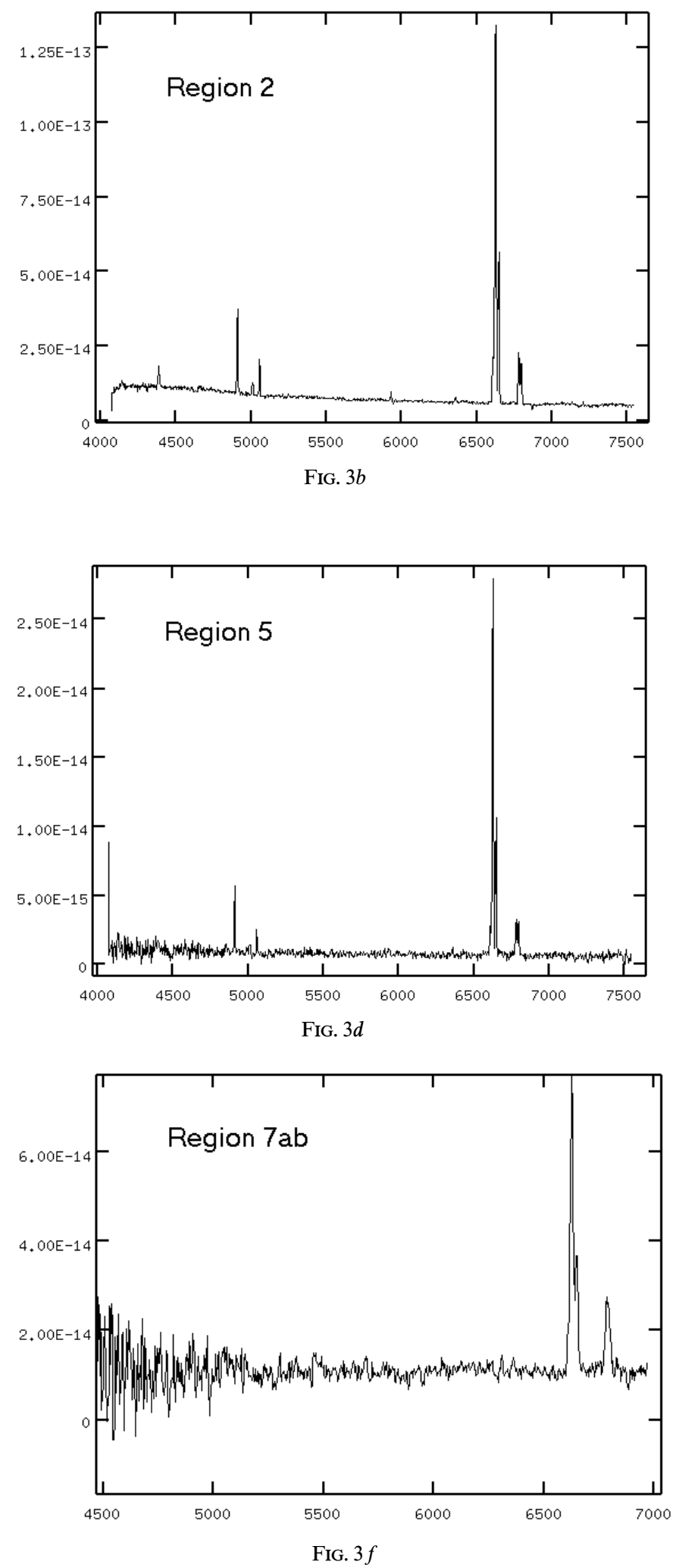

FIG. 3.- Optical spectra of six giant H II regions, located in the nuclear and central area of NGC 3256, plus the UV and near-IR spectra of the circumnuclear area (see the text).

nosities in $\mathrm{H} \alpha$ compatible with nuclear starburst (Table 4 and Kennicutt, Keel, \& Blaha 1989); (3) in the central area only the regions $7 \mathrm{a}$ and $7 \mathrm{~b}$ (arm III) show some differences in the spectra, i.e., a strong reddening in the continuum and the emission lines.
In particular, the observed spectra in the three asymmetrical spiral arms and in R1 and R2 (Figs. $3 a-3 i$; Table 4) show-practically all - the typical features of low-ionization and relatively high metallicity giant $\mathrm{H}$ is regions (Diaz et al. 1987, 1991; Terlevich 1993; Garcia Vargas \& Diaz 1994): 

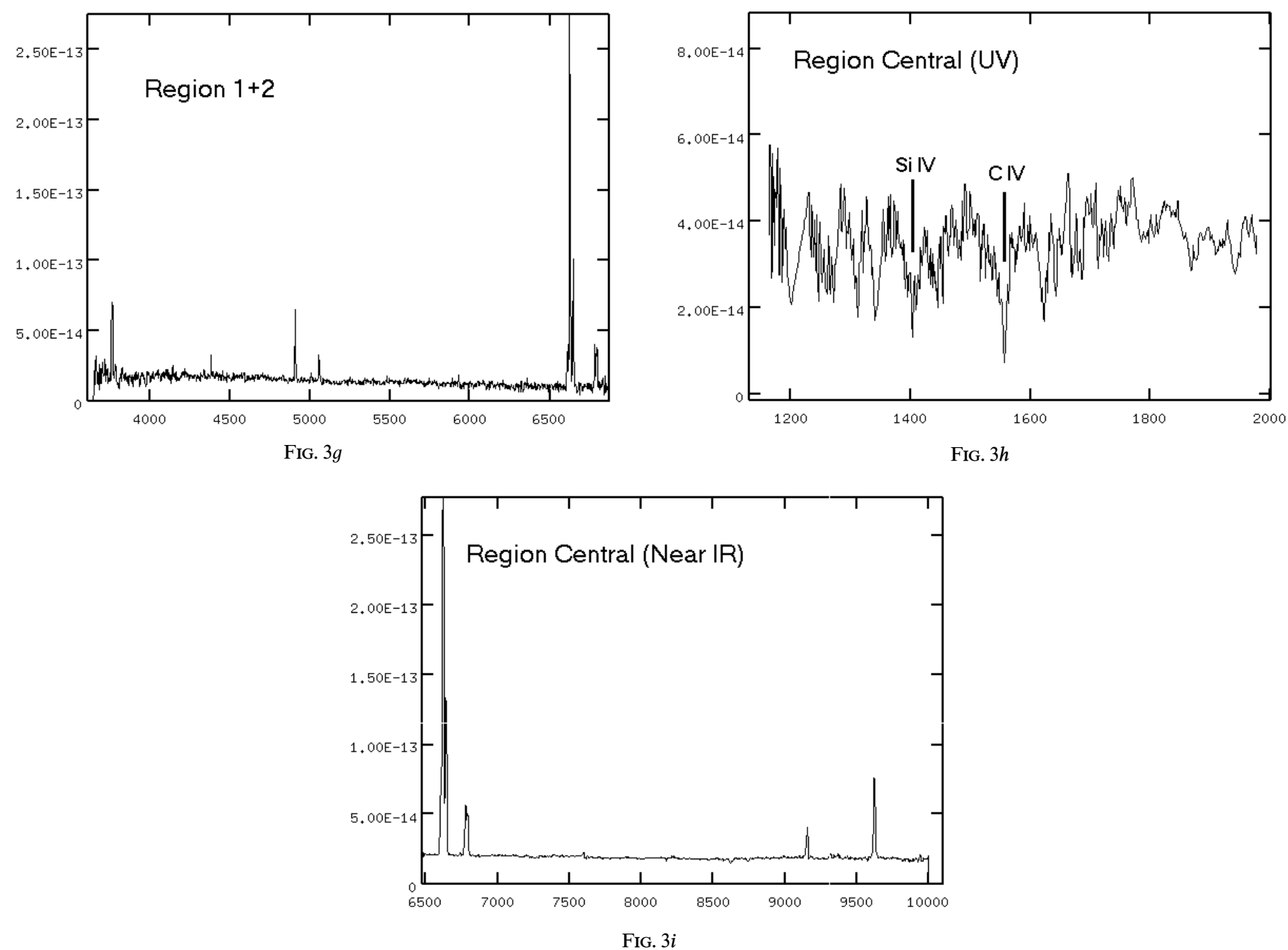

very low $[\mathrm{O} \mathrm{III}] \lambda 5007 / \mathrm{H} \beta$ ratio $(\sim 0.5)$, and absent or very weak [O III] $\lambda 4363$ and $[\mathrm{N}$ II $] \lambda 5755$.

On the other hand, in order to study the stellar populations, it is also important to analyze the absorption spectra of NGC 3256. Table 6 shows the equivalent width (EW)- corrected by galactic reddening - for the main UV, optical, and near-IR absorption lines (from spectra obtained at CTIO, CASLEO, and IUE; Figs. $3 a-3 f$ ). We measured for the region $1+2$-in the near-IR - the EW of the absorption triplet of $\mathrm{Ca}$ II (Ca II $\lambda \lambda 8498,8542,8662) ~ 1.1,5.4$, and

TABLE 5

EMISSION-LiNe Ratios of NGC 3256 aNd NGC 3256C

\begin{tabular}{|c|c|c|c|c|c|c|}
\hline Regions & $\log [\mathrm{O} \mathrm{III}] / \mathrm{H} \beta^{\mathrm{a}}$ & $\log \left[\mathrm{O}_{\mathrm{I}}\right] / \mathrm{H} \alpha^{\mathrm{a}}$ & $\log [\mathrm{N} \mathrm{II}] / \mathrm{H} \alpha^{\mathrm{a}}$ & $\log [\mathrm{S} I I] / H \alpha^{a}$ & $\log [\mathrm{O} \mathrm{II}+\mathrm{O} \mathrm{III]}] / \mathrm{H} \beta^{a}$ & $\mathrm{~S}$ II $/ \mathrm{S} \mathrm{II}^{\mathrm{a}}$ \\
\hline \multirow[t]{2}{*}{$\mathrm{R} 1^{\mathrm{b}} \ldots \ldots \ldots \ldots \ldots$} & -0.47 & -1.50 & -0.27 & -0.60 & $\ldots$ & 0.96 \\
\hline & {$[-0.50]$} & {$[-1.47]$} & {$[-0.28]$} & {$[-0.63]$} & $\ldots$ & \\
\hline \multirow{2}{*}{$\mathrm{R} 2+\ldots \ldots \ldots \ldots \ldots$} & -0.38 & -1.60 & -0.40 & -0.60 & $\ldots$ & 1.10 \\
\hline & {$[-0.41]$} & {$[-1.57]$} & {$[-0.40]$} & {$[-0.62]$} & $\ldots$ & \\
\hline \multirow[t]{2}{*}{$\mathrm{R} 4 \ldots \ldots \ldots \ldots \ldots$} & -0.27 & -1.44 & -0.44 & -0.57 & $\ldots$ & 1.23 \\
\hline & {$[-0.30]$} & {$[-1.42]$} & {$[-0.45]$} & {$[-0.58]$} & $\ldots$ & \\
\hline \multirow[t]{2}{*}{ R5 ............. } & -0.30 & -1.30 & -0.43 & -0.66 & $\ldots$ & 1.30 \\
\hline & {$[-0.33]$} & {$[-1.27]$} & {$[-0.43]$} & {$[-0.68]$} & $\ldots$ & \\
\hline \multirow{2}{*}{ R6 ............... } & -0.33 & -1.42 & -0.38 & -0.50 & $\ldots$ & 1.24 \\
\hline & {$[-0.36]$} & {$[-1.38]$} & {$[-0.38]$} & {$[-0.53]$} & $\ldots$ & \\
\hline \multirow[t]{2}{*}{$\mathrm{R} \mathrm{ab}^{\mathrm{c}} \ldots \ldots \ldots \ldots$} & $(-0.38)$ & $(-1.30)$ & -0.37 & -0.39 & $\ldots$ & 1.34 \\
\hline & {$[(-0.41)]$} & {$[(-1.26)]$} & {$[-0.38]$} & {$[-0.42]$} & $\ldots$ & \\
\hline \multirow{2}{*}{$\mathrm{R} 1+\mathrm{R} 2 \ldots \ldots \ldots \ldots$} & -0.36 & -1.54 & -0.41 & -0.56 & 0.33 & 0.98 \\
\hline & {$[-0.37]$} & {$[-1.52]$} & {$[-0.42]$} & {$[-0.56]$} & {$[0.44]$} & \\
\hline NGC $3256 C \ldots . .$. & -0.25 & -0.82 & -0.36 & -0.37 & $(0.65)$ & 1.40 \\
\hline
\end{tabular}

${ }^{a}[\mathrm{O}$ III $] \lambda 5007 ;[\mathrm{O} \mathrm{I}] \lambda 6300 ;[\mathrm{N} \mathrm{II}] \lambda 6583 ;$ [S IIS $] \lambda \lambda 6716+6731 ;[\mathrm{O} \mathrm{II}] \lambda 3727 ;[\mathrm{S} \mathrm{II}] /[\mathrm{S} \mathrm{II}] \lambda \lambda 6716 / 6731$.

b Values in square brackets are corrected by internal reddening [the color excess $E(B-V)_{I}$ values were obtained from Table 4].

${ }^{c}$ The values between parentheses are data with low $\mathrm{S} / \mathrm{N}$. 
TABLE 6

EQUivalent WidTh of THE AbSORPTION LiNeS OF NGC 3256

\begin{tabular}{|c|c|c|}
\hline Absorption Lines & $\mathrm{EW}^{\mathrm{a}}(\AA)$ & Comments \\
\hline \multicolumn{3}{|c|}{ Optical } \\
\hline \multicolumn{3}{|l|}{ Regions $1+2$ (CTIO): } \\
\hline $\mathrm{Mg} \mathrm{I}+\mathrm{H} 9 \lambda_{3835} \ldots \ldots \ldots \ldots \ldots$ & 1.7 & \\
\hline Ca II K 23933 ................... & 6.0 & \\
\hline Ca II H $\lambda 3968 \ldots \ldots \ldots \ldots \ldots \ldots$ & 4.1 & \\
\hline \multicolumn{3}{|l|}{ Regions $1,2,6,7$ (CASLEO): } \\
\hline Na I D $\lambda 58900$ Reg $1 \ldots \ldots \ldots . .$. & 4.1 & \\
\hline Na I D $\lambda 5890 \operatorname{Reg} 2 \ldots \ldots \ldots \ldots$ & 2.6 & Absorption + emission \\
\hline I D $\lambda 5890 \operatorname{Reg} 6 \ldots \ldots \ldots \ldots \ldots$ & 5.5 & Absorption + emission \\
\hline Na I D $\lambda 5890 \operatorname{Reg} 7 \ldots \ldots \ldots . .$. & 5.0 & Absorption + emission \\
\hline \multicolumn{3}{|c|}{ UV and Near-IR } \\
\hline Regions $1+2(I U E-$ CTIO): & & \\
\hline Si Iv $\lambda 1400 \ldots \ldots \ldots \ldots \ldots \ldots \ldots \ldots \ldots \ldots \ldots \ldots$ & 10.0 & $\lambda_{\text {obs }}=1403.5 \AA$ \\
\hline $\mathrm{C}$ iv $\lambda 1550 \ldots \ldots \ldots \ldots \ldots \ldots \ldots$ & 13.0 & $\lambda_{\mathrm{obs}}=1555.5 \AA$ \\
\hline $\mathrm{Fe}$ III $+\mathrm{Al}$ III $\lambda \lambda 1600-1630 \ldots . .$. & 3 & Absorption blend \\
\hline He II $\lambda 1640 \ldots \ldots \ldots \ldots \ldots \ldots$ & (4) & Absorption + emission \\
\hline Ca II $\lambda 8498$. & 1.1 & \\
\hline Ca II $28542 \ldots \ldots \ldots \ldots \ldots \ldots \ldots$ & 5.4 & \\
\hline Ca II $\lambda 8662 \ldots \ldots \ldots \ldots \ldots \ldots$ & 3.4 & \\
\hline
\end{tabular}

${ }^{a}$ The spectra are corrected for atmospheric extinction, galactic reddening $[E(B-V)=0.13]$, and redshift (see the text).

$3.4 \AA$, respectively, and these lines were not detected as emission features (see the discussion of these results in $\S 4.4$ ).

The clearer "signature" of massive stars and starburst is the presence of absorption lines at the UV region of the spectrum (see Weedman et al. 1981; Leitherer 1991). From the UV-IUE library of Seyfert and starburst galaxies (McQuade, Calzetti, \& Kinney et al. 1995; StorchiBergmann et al. 1995) we have obtained UV spectra of NGC 3256, in the range 1200-2000 ̊. This spectrum shows mainly, for the circumnuclear region $\left(10^{\prime \prime} \times 20^{\prime \prime}\right.$; Fig. $\left.3 g\right)$, the typical starburst broad absorption lines: Si IV $\lambda 1400$ and $\mathrm{C}$ IV $\lambda 1550$. These lines show profiles very similar to the prototype of starburst galaxy NGC 7714 (Weedman et al. 1981). We measured the equivalent width ratio (EW) of these lines, and we found $\mathrm{EW}(\mathrm{Si} \mathrm{IV}) / \mathrm{EW}(\mathrm{C}$ IV) $=$ $0.76 \pm 0.06$, using the spectral windows of $\lambda \lambda 1380-1415$ and $\bar{\lambda} \lambda 1523-1576$, respectively (already used by StorchiBergmann et al. 1995 and Robert, Leitherer, \& Heckman 1993 for their analysis of UV-IUE data). For lowmetallicity systems, the $\mathrm{EW}(\mathrm{Si} \mathrm{IV}) / \mathrm{EW}(\mathrm{C}$ IV) ratio is an important parameter in order to study the initial mass function (IMF); however, for objects with solar abundancessuch as NGC 3256 - a detailed study of the interstellar contribution is required (since the effect of the interstellar material increase with metallicity). The value of the $\mathrm{EW}(\mathrm{Si} \mathrm{IV}) / \mathrm{EW}(\mathrm{C}$ IV) is in agreement with the average obtained for starburst galaxies: $0.69 \pm 0.13$ (Sekiguchi \& Anderson 1987; Scalo 1990) and $0.83 \pm 0.1$ (Robert et al. 1993 ; see $\S 4.4$ ).

It is important to note, that the two main "broad" absorption lines show - in the IUE spectra - a strong blueshift of $V_{\mathrm{Si} \mathrm{IV}}=-750 \pm 60 \mathrm{~km} \mathrm{~s}^{-1}$ and $V_{\mathrm{C}_{\text {IV }}}=-1070 \pm 40$ $\mathrm{km} \mathrm{s}^{-1}$ (see Table 6), in agreement with the prediction of starburst models in which the Si IV and C IV absorption lines can be formed mainly by stellar winds with a P Cyg profile (Robert et al. 1993) and also by the photosphere of B stars (in the case of Si IV) and by the interstellar medium
(ISM). Furthermore, in the UV region, NGC 3256 shows a composite spectra, showing features typical of starburst (e.g., NGC 7714), strong Si Iv, C Iv, and He II lines, and also of IR galaxies (e.g., NGC 7552) with strong $\mathrm{Fe}$ III + Al III blend at $1600-1630 \AA$ and many weak absorption lines from young stars, plus a strong reddening in the UV continuum. Additionally, no shift is expected for the Al III and Fe III lines, since they are originate in the ISM and in the photosphere of the stars (Robert et al. 1993).

\subsubsection{Optical Spectrophotometry with High Spectral/Spatial Resolution}

The relatively high spectral and spatial resolution $(90$ $\mathrm{km} \mathrm{s}^{-1}$ and $\sim 0$ ".8 sampling) allowed the study of the spatial variation of emission lines, physical conditions, and ionizing sources in NGC 3256. In particular, it is important to know the physical conditions in the nuclei, the spiral arms, the outflow component, and the more external regions.

First, we present in Figures $4 a-4 h$ the spatial variation of the emission line ratios [N II $] \lambda 6584 / \mathrm{H} \alpha$, [S II $] \lambda 6731 /[\mathrm{S}$ II $]$ $\lambda 6717,[\mathrm{~S} \mathrm{II}] \lambda \lambda 6717+6731 / \mathrm{H} \alpha$, and the FWHM of the $\mathrm{H} \alpha$ emission line $[\mathrm{FWHM}(\mathrm{H} \alpha)]$ for seven selected P.A.'s $\left(70^{\circ} /\right.$ $56^{\circ}, 155^{\circ} / 18^{\circ}, 45^{\circ} / 155^{\circ}$, and $65^{\circ} / 40^{\circ}$ ). These figures show that (1) the ratio [N II] $\lambda 6584 / \mathrm{H} \alpha$ decreases from 0.60 in the main optical nucleus (R1) and 0.80 in several outer regions to 0.30 in the circumnuclear areas; (2) the ratio [S II] $\lambda 6731 / \mathrm{S}$ II] $\lambda 6717$ decreases from 1.08 in the main nucleus and 0.95 in the spiral arms to $0.6-0.70$ in the external areas; (3) the ratio [S II] $\lambda \lambda 6717+6731 / \mathrm{H} \alpha$ increases from 0.20 0.30 in the central regions to $0.40-0.60$ in the outer regions (for P.A.'s inside the outflow bipolar cone; e.g., P.A. $155^{\circ}$, Fig. $4 f$ ); and it is constant $-0.20-0.30$ - for P.A.'s outside of this outflow area (e.g., for P.A. of $45^{\circ}$, Fig. 4e); (4) the $\operatorname{FWHM}(\mathrm{H} \alpha)$ increases from the instrumental profile value of $\sim 90 \mathrm{~km} \mathrm{~s}^{-1}$ in the outer regions to $\sim 270 \mathrm{~km} \mathrm{~s}^{-1}$ in the lobe with high blue velocity values and arm III, and for the two main optical knots (R1 and R2), the FWHM(H $\alpha)$ values reach $\sim 220 \mathrm{~km} \mathrm{~s}^{-1}$.

In Figures $5 a-5 d$ we also display detailed maps of emission-line ratios [N $\mathrm{II}] \lambda 6584 / \mathrm{H} \alpha$, [S $\mathrm{II}] \lambda 6731 /$ [S II] $]$ 6717, [S II] $\lambda \lambda 6717+6731 / \mathrm{H} \alpha$, and $\mathrm{FWHM}(\mathrm{H} \alpha)$ for the central region of NGC $3256\left(\sim 30^{\prime \prime} \times 30^{\prime \prime}\right.$ with $1^{\prime \prime}$ spatial resolution). These maps were made using a technique similar to that described in $\S 3.2$, for the case of the velocity field map. It is important to note that these maps were made for the main component of the emission lines. We remark the following:

1. In the central $20^{\prime \prime} \times 20^{\prime \prime}\left(r \leq 13^{\prime \prime} \sim 2 \mathrm{kpc}\right)$ all the emission-line ratios maps show-for the main component - the typical values of giant $\mathrm{H}$ II regions (see Terlevich et al. 1991; Kennicutt et al. 1989; and references therein).

This result is in agreement with the fact that this is the area of strongest emission lines (Figs. $1 c, 1 d$, and 1e) coming mainly from the nuclei and the asymmetrical spiral arms (where we detected ongoing massive star formation process; see $\$ \S 3.3 .1$ and Fig. 3).

2. The [S II] $\lambda \lambda 6717+6731 / \mathrm{H} \alpha$ map shows for $r \geq 10^{\prime \prime}$ the typical values of ionization by shock heating ([S II] $\lambda \lambda 6717+6731 / \mathrm{H} \alpha \geq 0.32$; HAM90).

There are two main extended regions in which this ratio reaches very high values $(0.40-0.60)$, aligned with the same P.A. of the outflow component axis (§ 3.2.2; P.A. $\left.\sim 150^{\circ}\right)$. 


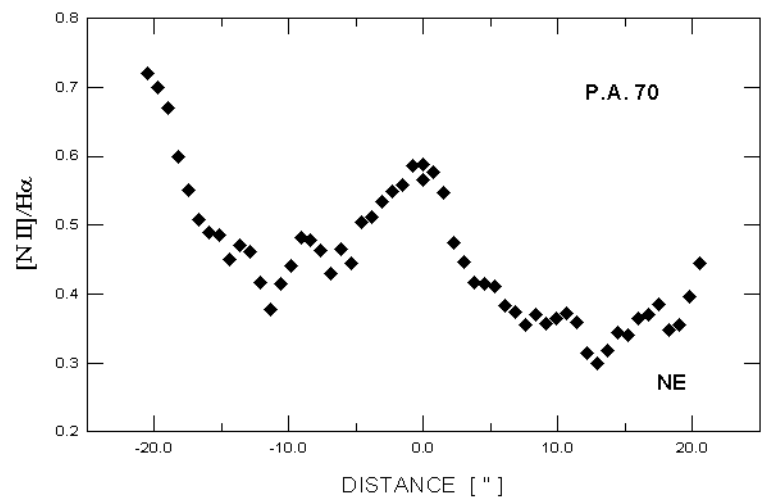

FIG. $4 a$

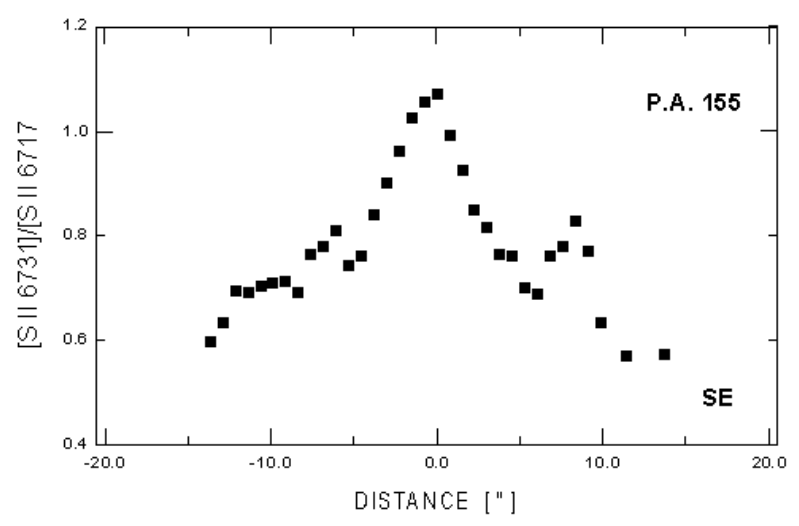

FIG. $4 c$

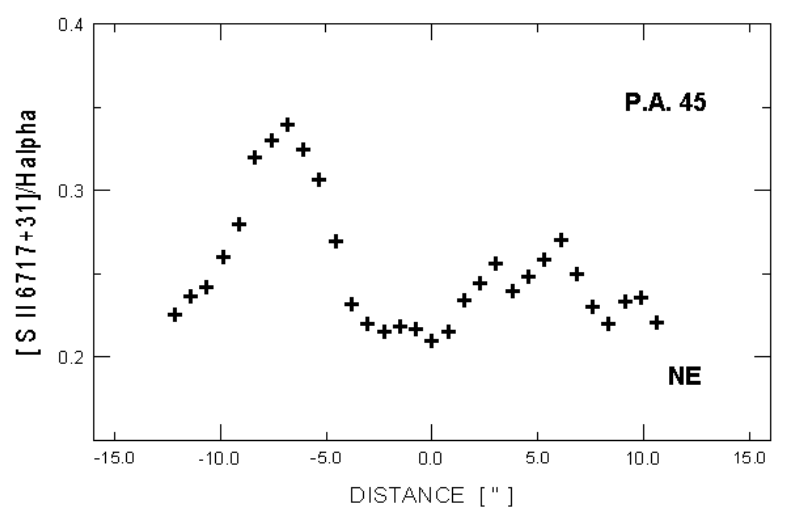

FIG. $4 e$

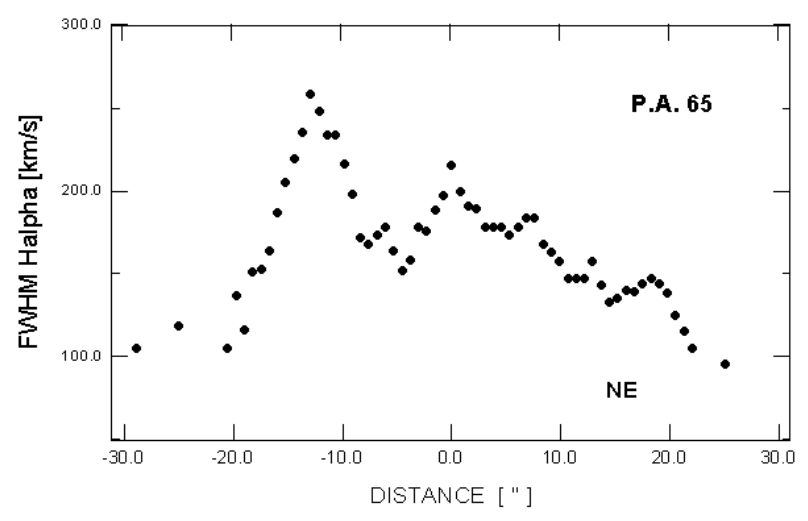

FIG. $4 g$

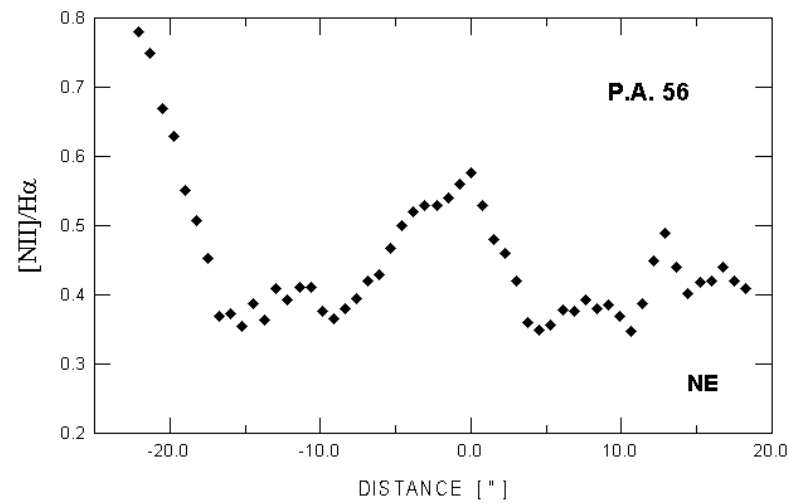

FIG. $4 b$

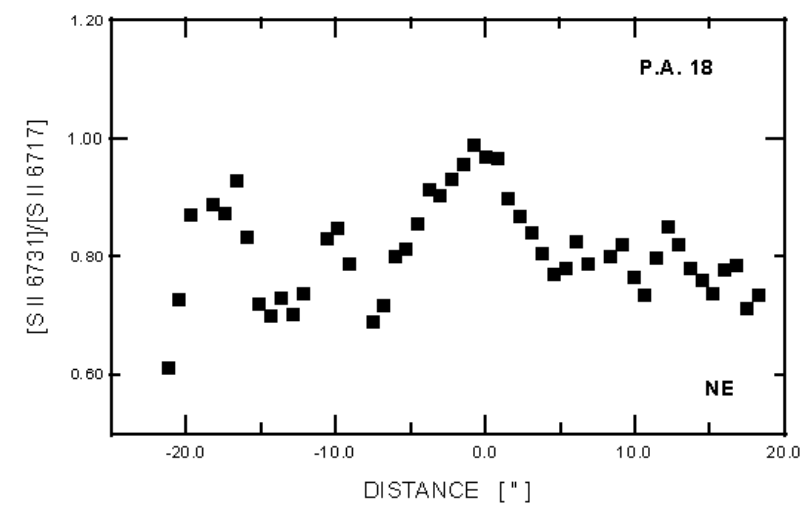

FIG. $4 d$

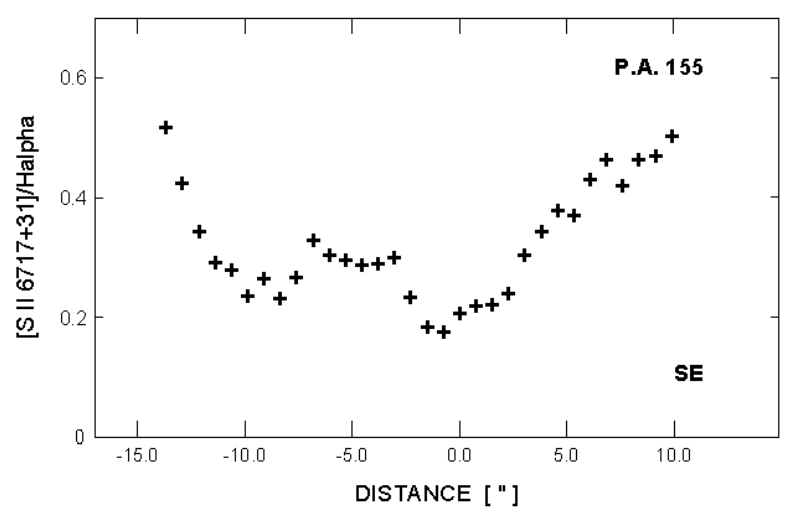

FIG. $4 f$

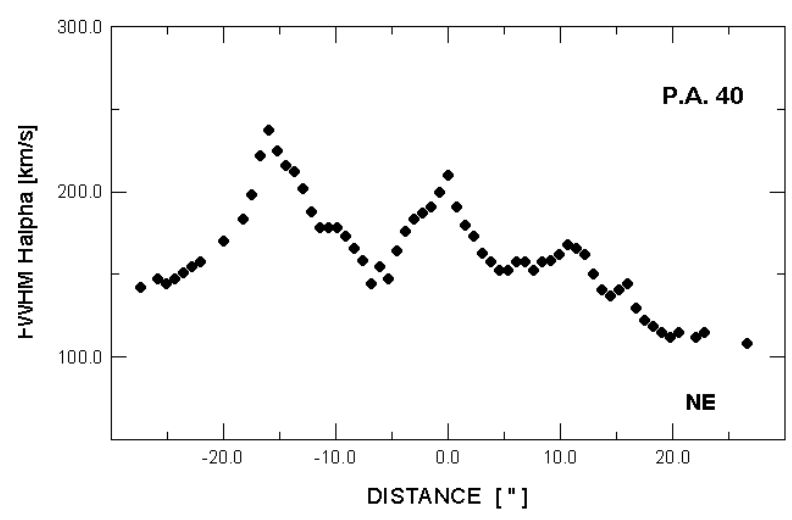

FIG. $4 h$

FIG. 4.-Emission-line ratios and $\operatorname{FWHM}(\mathrm{H} \alpha)$ vs. axial distance from the main optical nucleus (along P.A. $=70^{\circ}, 56^{\circ}, 155^{\circ}, 18^{\circ}, 45^{\circ}, 155^{\circ}, 65^{\circ}$, and $^{\circ} 0^{\circ}$ ). 

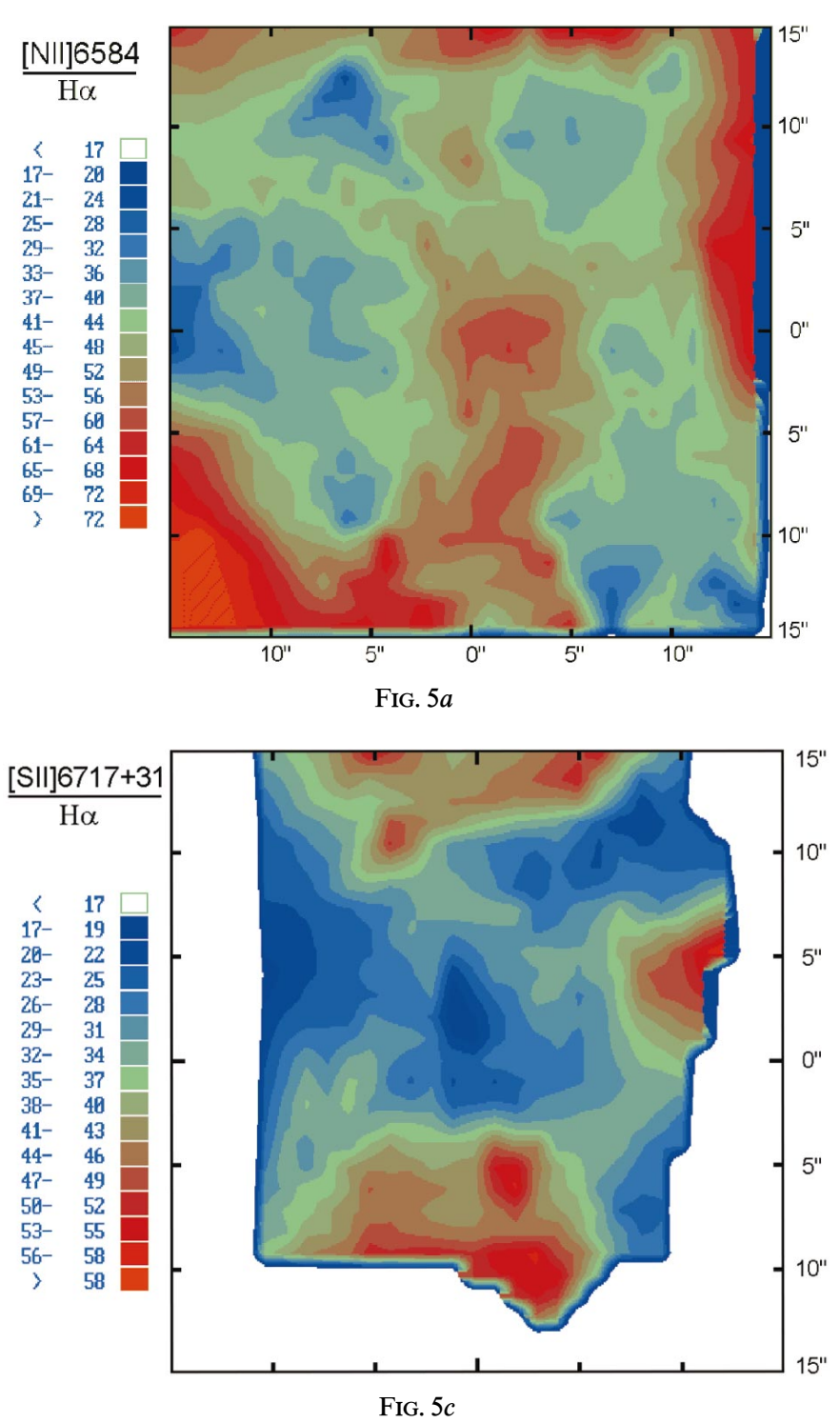
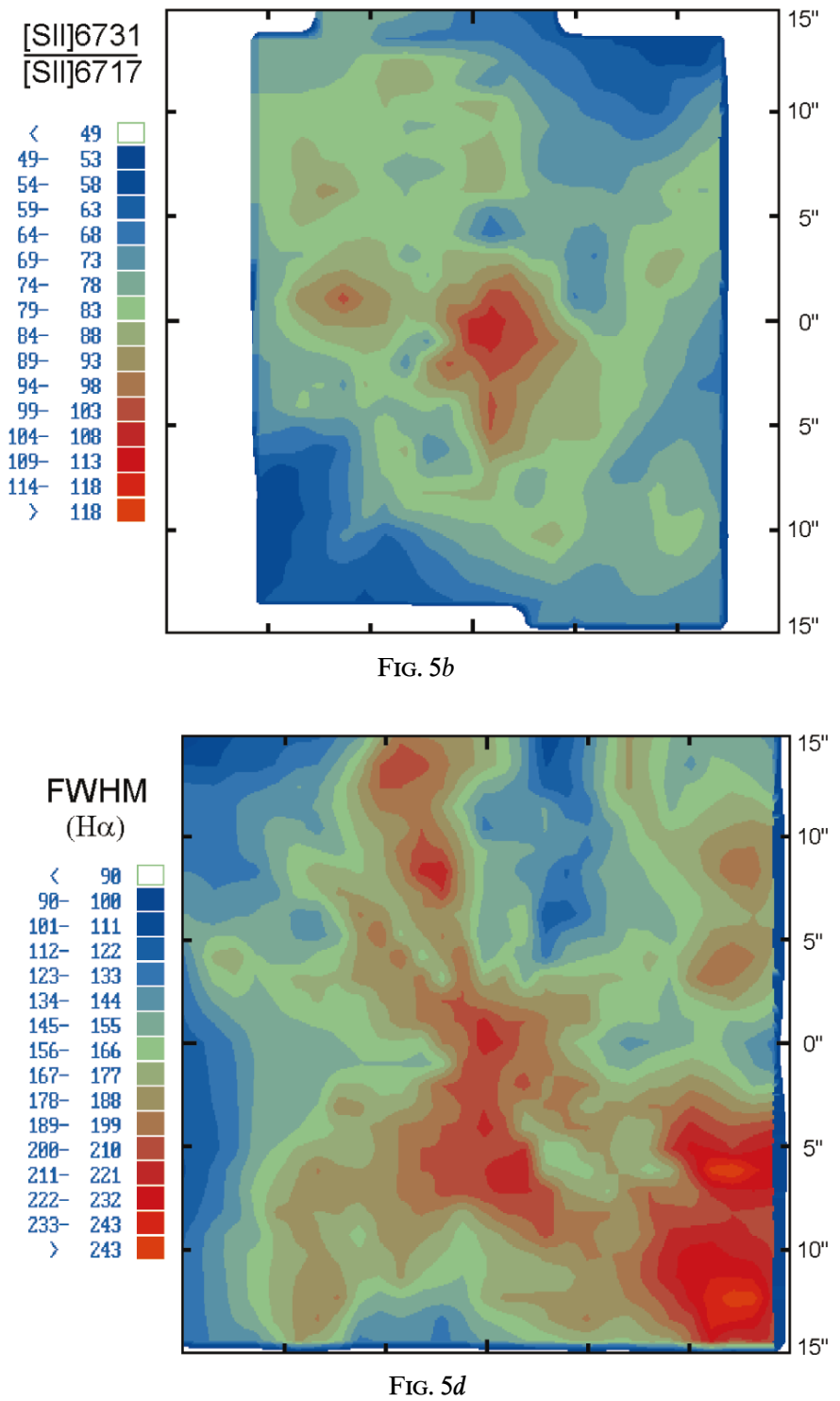

FIG. 5.-Emission-line ratios and $\operatorname{FWHM}(\mathrm{H} \alpha)$ color maps, for the central regions and the main/strong component. North is up, and east is to the left (the scale of the emission-line ratios includes a factor of $10^{2}$ ).

The other extended region that shows high values of this $[\mathrm{S} \mathrm{II}] / \mathrm{H} \alpha$ ratio is located close to the third source of polarization (Scarrott et al. 1996).

3. The [S II] $\lambda 6731 /[\mathrm{S}$ II] $\lambda 6717$ map shows two strong lobes at the position of the two main optical nucleus (reaching values in the range 1.00-1.10), with an elongated lobe in the area of R3. There are also secondary peaks (reaching values of 0.94-0.98) in the other circumnuclear giant $\mathrm{H}$ II regions: $\mathrm{R} 4, \mathrm{R} 6$, and $\mathrm{R} 9$.

4. The $[\mathrm{N} \mathrm{II}] \lambda 6584 / \mathrm{H} \alpha$ map shows a complex structure, with high values in the nuclear and external regions. This complex shape could be the result of the superposition of two effects: overabundance of $\mathrm{N}$ (in the nuclear starburst regions, where the wind of massive Wolf-Rayet stars and/or luminous young globular clusters contributes to generate overabundance of N; see Kobulnicky et al. 1997) and shock heating.

It should be noted, that in the "central regions," the $[\mathrm{N} \mathrm{II}] / \mathrm{H} \alpha$ ratio and $\mathrm{FWHM}(\mathrm{H} \alpha)$ maps show a nice spatial correlation, i.e., the extended areas of high values - in each map - show a similar location. Furthermore, these areas display a similar elongated shape, and they are inside the bipolar outflow region (and close to the direction of the axis of the outflow cone). This result indicates the places where the ionization is produced by shock heating, which broadens the emission lines (see $\S 4.5$, Storchi-Bergmann, Wilson, \& Baldwin 1996 and Lípari et al. 1997 for discussions related to the role of shock processes in the origin of high values of the $[\mathrm{N} \mathrm{II}] / \mathrm{H} \alpha$ ratio).

5. There are two strong peaks in the $\operatorname{FWHM}(\mathrm{H} \alpha)$ map, associated with the main knots in arm III (R7) and with the area of very high negative velocity values. In addition, this map shows secondary peaks at the position of R1 and also in several central and outer regions (Fig. $5 d$ ). This increase in the $\operatorname{FWHM}(\mathrm{H} \alpha)$ could be explained by the presence of multiple components besides the main/strong component (with different velocities) plus shock processes.

These results strongly suggest that the "dominant" sources of ionization, detected in the "main" emission-line component are (1) giant $\mathrm{H}$ II regions, in the nuclear and central area (located in the nuclei and the spiral arms); and 
(2) shock heating, in the central/external regions where we also found radial filaments (Fig. $1 b$ ) and extended outflow (Fig. 2f), at the same places where Scarrott et al. (1996) detected the more extended polarization field known (associated mainly with the outflow component). In addition, for the central region, we also detected composite ionization source at some places: $\mathrm{H}$ II regions plus shocks.

\subsubsection{Properties of the $\mathrm{H}$ in Regions and the Star Formation}

The obtained emission line ratios of [S II] $\lambda \lambda 6731 / 6717$ (Figs. 4 and 5) show a continuum variation in a range 1.10 0.95 , in the ionized gas. The highest value of the [S II] $\lambda \lambda 6731 / 6717$ ratio (i.e., the highest electron density condition, $N_{e}$ ) is reached in the main optical nucleus. Note the good agreement between these high-resolution data and those present in Tables 4 and 5 (which were obtained with moderate resolution and for six strong knots).

The corresponding range of $N_{e}$ obtained from the values of [S II] $\lambda \lambda 6717,6731$ ratio and the relation given by Osterbrock (1989) and Aller (1984) is $\sim 100-1000 \mathrm{~cm}^{-3}$. The radial dependence of the [S II] ratio and the density could be explained by the fact that in the nuclear regions there is a highest concentration of ionized gas.

In low-ionization giant $\mathrm{H}$ II regions and starburst (i.e., high-metallicity giant $\mathrm{H}$ II regions) the determination of electron temperature in the ionized gas $\left(T_{e}\right)$ and abundance is not straightforward, mainly owing to the absence of the lines [O III] $\lambda 4363$ and [N II] $\lambda 5755$. Therefore, the determination of the physical conditions (specially abundance) requires a detailed study in a wide spectral range $\lambda \lambda 3600$ 10000 (Pagel et al. 1979; Diaz et al. 1987, 1991; Vilches et al. 1988; Terlevich 1993; Diaz \& Perez-Montero 1999). A range of $6000-7000 \mathrm{~K}$ of ionized gas electron temperature $\left(T_{e}\right)$ was obtained, using the diagrams of Pagel et al. (1979), Dopita \& Evans (1986), and Aguero \& Lípari (1991).

In general the values of density and temperature in the ionized gas obtained in this paper (for different regions of NGC 3256) show a good agreement with those obtained previously for the whole central region of this galaxy. In particular, Storchi Bergmann et al. (1995) found for the circumnuclear region $\left(10^{\prime \prime} \times 20^{\prime \prime}\right.$, aperture) a value of $N_{e}=$ $204 \mathrm{~cm}^{-3}$ and $T_{e}=6162 \mathrm{~K}$. Additionally, Aguero \& Lípari (1991) found for the nuclear region $\left(3^{\prime \prime} \times 6^{\prime \prime}\right.$ aperture) a value of $N_{e}=600 \mathrm{~cm}^{-3}$ and $T_{e}=5900 \mathrm{~K}$. In addition, these results are in agreement with the upper limit of $N_{e} \sim 1400$ $\mathrm{cm}^{-3}$ reported from Carral et al. (1994) based in their measurement of the [O III] $(88 / 52 \mu \mathrm{m})$ IR line ratio.

There are different and detailed methods to calculate the physical conditions of the ionizing source in giant $\mathrm{H}$ II regions (from optical emission lines), mainly using photoionization codes plus starburst model (Garcia Vargas et al. 1997; Garcia Vargas \& Diaz 1994; Garcia Vargas et al. 1995a, 1995b; Leitherer \& Heckman 1995). First, we measured the temperature of the ionizing source using the optical emission-line ratios (Table 5) and the results of photoionization and $\mathrm{H}$ II region/star cluster models of Pastoriza et al. (1993) for NGC 3310, and we obtained in all their optical and near-IR diagrams (for the six regions) a value of the ionizing source $T_{\text {eff }} \sim 35,000 \mathrm{~K}$ (their Figs. $6 a, 6 b, 6 c$, and $6 d$; Pastoriza et al. 1993), for a parameter of ionization $\log (U)=-3.0$ and solar abundance. In particular, using abundance-dependent diagrams, $[\mathrm{N} \mathrm{II}] \lambda 6584 / \mathrm{H} \alpha$ versus $([\mathrm{O}$ II $] \lambda 3727+[\mathrm{O}$ III $] \lambda 5007) / \mathrm{H} \beta$, and $([\mathrm{S} \mathrm{II}] \lambda \lambda 6717+6731+[\mathrm{S}$ III $] \lambda 9532) / \mathrm{H} \beta$ versus $([\mathrm{O}$ II $]$ $\lambda 3727+[\mathrm{O}$ III $] \lambda 5007) / \mathrm{H} \beta$ (their Figs. $6 c$ and $6 d$ ), we found metallicity close to solar abundance.

These results are in excellent agreement with those obtained by Joseph (1991) and Doyon, Joseph, \& Wright (1994) from measurement of IR emission lines (He I, 2.06 $\mu \mathrm{m} ; \mathrm{Br} \gamma, 2.17 \mu \mathrm{m}$; and $\mathrm{H}_{2}$ ) and IR absorption lines (CO band longward of $2.3 \mu \mathrm{m}$; Na I doublet, $2.206 \mu \mathrm{m}$; and Ca I triplet, $2.263 \mu \mathrm{m}$ ). They found for this powerful starburst $T_{\text {eff }} \sim 35,000 \mathrm{~K}$, lower mass cutoff $\sim 3-6 M_{\odot}$, upper mass cutoff $\sim 30 M_{\odot}$, and age 12-27 $\times 10^{6}$ yr (using a deduced IMF slope of $\alpha \leq 2.2$, where $\alpha$ comes from $d N(m) /$ $d m \sim m^{-\alpha}$ ). They used the starburst models of Telesco \& Gatley (1984) and Prestwich, Joseph, \& Wright (1994). In addition, Moorwood \& Oliva (1994) found practically the same parameters for the starburst in NGC 3256, also using near-IR emission-lines data ([Fe II $] 1.64 \mu \mathrm{m}, \mathrm{H}_{2} 2.12 \mu \mathrm{m}$, $\mathrm{Br} \gamma 2.17 \mu \mathrm{m})$; in particular, they obtained a lower mass cutoff $\sim 6 M_{\odot}$, an upper mass cutoff $\sim 30 M_{\odot}$, and an IMF slope $\alpha \sim 2.5$.

Recently, Rigopoulou et al. (1996) and Lutz et al. (1996) studied the starburst/dust of NGC 3256, using new Infrared Space Observatory (ISO) Short Wavelength Spectrometer (SWS) near- and mid-IR emission-line observations ([Ne III]/[Ne II $](15 / 12 \mu \mathrm{m}),[\mathrm{Ar}$ III]/[Ar II $](8.9 / 6.9 \mu \mathrm{m}),[\mathrm{S} \mathrm{IV}] /[\mathrm{S}$ III] $(10.5 / 18.7 \mu \mathrm{m}))$, plus new Sellmaier et al. (1996), Kurucz (1992), and Kobo \& Sternberg (1996) stellar atmosphere and starburst models. They found for the ionizing radiation field (in the starburst $/ \mathrm{H}$ II regions) $T_{\text {eff }} \sim 41,000 \pm 3000 \mathrm{~K}$, upper mass cutoff $\sim 50-100 M_{\odot}$, and age $10-20 \times 10^{6} \mathrm{yr}$ [for $\log (U)=-2.5$, solar abundance, and Salpeter IMF with slope of 2.4]. They suggest that the formation of very massive stars is probably not inhibited in NGC 3256. In $\S 4.4$ we analyze this interesting result.

The abundances were calculated following the empirical method of Pagel et al. (1979) and Diaz \& Perez-Montero $(1998,1999)$ for low-excitation $\mathrm{H}$ II regions, and we derived for the $\mathrm{R} 1+\mathrm{R} 2: \mathrm{O}^{+} / \mathrm{H}^{+}=8.7, \mathrm{O}^{++} / \mathrm{H}^{+}=8.1, \quad \mathrm{~N}^{+} /$ $\mathrm{H}^{+}=7.9, \mathrm{~S}^{+} / \mathrm{H}^{+}=6.7, \mathrm{O} / \mathrm{H}=8.8, \mathrm{~S} / \mathrm{H}=6.6$. These are similar values to those obtained by Aguero \& Lípari (1991) and Storchi-Bergmann et al. (1995) for the whole circumnuclear region. Therefore, the higher ratio $[\mathrm{N}$ II $] \lambda 6584 / \mathrm{H} \alpha$ observed in the nuclear region is probably due to high metallicity plus shock excitation.

\subsection{The NGC 3256 Group of Galaxies}

We describe here the environment of NGC 3256 and its group, mainly for the following reasons: (1) NGC 3256C, the closer galaxy to NGC 3256 (at $\sim 150 \mathrm{kpc}$ and approximately $-100 \mathrm{~km} \mathrm{~s}^{-1}$ ) is also an ongoing merger, and these two systems plus the other members of the group could evolve to mergers between mergers; (2) in this group (and also in the close NGC 3263 group) practically all the members show interacting/merger morphology (e.g., NGC 3256A shows extended tidal tails); (3) the number of members in NGC 3256 group is not clearly defined; in particular two works based in the same sample of galaxies (Fouque et al. 1992; Garcia 1993) obtained different results, using a similar computational algorithm; (4) the ESO NTTEMMI images show clearly in the southern part of NGC 3256 two dwarf galaxies (Fig. $1 b$ ) at a projected distance of $\sim 100^{\prime \prime}-120^{\prime \prime}$, from the nucleus, and recently, similar dwarf galaxies were found close to Arp 220 by Ohyama et al. (1999). Also, Mould et al. (1991) studied the probable presence of a cluster of galaxies around the NGC 3256 group, and they found a high number of members for this cluster. 
We are working on a detailed study (imaging, kinematics, and spectrophotometry) of the individual members and members candidates of the NGC 3256 and NGC 3263 groups of galaxies, in a radius of $1^{\circ}$. In particular, we started with the study of the galaxies NGC 3256C, NGC 3256A, NGC 3256B, NGC 3261, NGC 3366, NGC 3263/3262, and ESO 317-G-20 (Lípari et al. 2000b). In general, we found (1) that NGC 3256C and NGC 3256A show merger morphology; specifically NGC $3256 \mathrm{C}$ shows similar structure to the merger NGC 3310 (Pastoriza et al. 1993); (2) the nuclear spectra of NGC 3256C (Table 5) show emission line ratios located - in the diagnostic diagrams - in the area between low-ionization $\mathrm{H}$ in regions and LINERs; (3) the circumnuclear $\mathrm{H}$ II regions of NGC $3256 \mathrm{C}$ show very strong blue continuum; and (4) NGC 3263 and NGC 3262 show also interacting structure, plus a tidal tail.

\section{DISCUSSION}

In this section we discuss the main new results obtained in $\S 3$, and a more detailed discussion (including models for multiple merger, for asymmetrical spiral arms, and extended massive star formation process) will be published elsewhere (Lípari et al. 2000b).

\subsection{Multiple Merger Model for NGC 3256 (and NGC 3256/3263 Groups)}

NGC 3256 is probably the advanced merger with more clear features indicative of "multiple" collision/merger. These main evidences came from the presence of (1) three compact nuclear knots, with morphological, kinematic, and spectroscopic properties of nuclear starburst; and (2) the presence of three spiral arms of massive star formation, which emanate from the three nuclei. In addition, the close presence of the merger NGC 3256C (at $150 \mathrm{kpc}$ and approximately $-100 \mathrm{~km} \mathrm{~s}^{-1}$ ) suggests the possibility - in the future - of mergers between mergers. These facts strongly suggest that NGC 3256 is the result of a collision between three galaxies. Such a multiple merger model has been proposed by Barnes (1989), Weil \& Hernquist (1996), and more recently by Taniguchi \& Shioya (1998).

In relation to the three nuclei, it is important to remark that (1) the regions 1 and 3 were previously confirmed - at radio and mid-IR wavelength - as two independent nuclei (by Norris \& Forbes 1995, Kotilainen et al. 1995, Zenner \& Lenzen 1993, and Moorwood \& Oliva 1994); and our VF shows that the kinematic center of NGC 3256 is located between these two regions; (2) our data show that R2 presents very similar morphological and spectroscopic properties to those found for R1 (the main optical nucleus); specifically, in $\S \S 3.1$ and 3.3, we found that both regions show similar nuclear starburst features (spectra, nuclear spiral disk, etc.). In addition, it is important to consider the nature of the third source of polarization (Scarrott et al. 1996). They suggested that probably it is a highly obscured nucleus, with a strong starburst. We found an offset of $\sim 1^{\prime \prime}$, between the third source of polarization and the nearest radio peak (Norris \& Forbes 1995), which is very weak and is located at the same position of the optical region 10 . Therefore, the third source of illumination in the polarimetry study could be associated with $\mathrm{H}$ in regions located in region 10 (however, our data do not exclude completely the presence of an obscured nucleus, in this area).
In $\S 3.1$, we found three independent asymmetrical spiral arms, in the central $5 \mathrm{kpc}$ of NGC 3256. It is important to note that these structures were detected using different images/wavelengths, for each arm: (1) the external part of arm 1 was clearly observed in the $\mathrm{H} \alpha$ NTT image, and the internal area in the HST WFPC2 broadband images; (2) the external and internal parts of arm 2 are evident in $H S T$ WFPC2 I broadband images; and (3) the HST NICMOS and $H S T /$ WFPC2 images show that arm 3 reaches R3. In $\mathrm{R} 2$, the nuclear spiral is less evident (than in R1), probably owing to the effects of inclination plus dust.

We consider - first - a not simultaneous multiple merger model for NGC 3256. Although the sense of rotation of arm I is the same as that of arm III, arm II has the opposite sense of rotation (with respect to those of arms I and III). Therefore, it is expected that the precursor group of galaxies of NGC 3256 consisted of three spiral galaxies, one of which (associated with R2) has a retrograde orbit, or its orbit has been modified during the course of successive mergers.

NGC 3256 has a pair of long tails in both eastern and western sides. The formation of these two tidal tails requires that the final merging between two galaxies occur as a prograde merger (e.g., Barnes \& Hernquist 1996, 1992 and references therein). However, one of these systems could be a previous merger between two objects. Therefore one possible scenario is (1) two galaxies associated with nucleus 1 and 2 merge at first with a retrograde orbit, and then (2) the merger remnant (between the two galaxies) merges with the remaining galaxy - associated with nucleus 3 - with a prograde orbit.

The size of the tails in NGC 3256 can give us a first estimation of the age of the merger, i.e., since the last major merger began. If an escape velocity of $100 \mathrm{~km} \mathrm{~s}^{-1}$ is assumed for the material in the tails (Colina et al. 1991) we obtain a value of $\sim 0.5 \times 10^{9}$ yr. This result is in good agreement with the timescale of mergers with prograde orbit: $0.5 \times 10^{9}$ yr (Barnes 1992; Noguchi 1991). It is important to note that the starburst activity detected in this merger must have occurred very late in the history of the interaction, since the age of the starburst is only 5-25 $\times 10^{6}$ $\mathrm{yr}(\S 3.3 .3)$. This is in agreement with the results published by Mihos \& Hernquinst (1996) for models of starbursts in disk/bulge/halo major merger processes (their Figs. 4 and 2 ), where the extended massive star formation occurred very late in the history of the interaction.

On the other hand, in the framework of the multiple merger scenario, an alternative model is a "simultaneous" triple merger (and for this case there are also several alternatives). A simple simultaneous model could be between two gas-rich massive spiral galaxies (Sc), and a satellite galaxy (of one of these Sc systems). In order to explore these types of scenarios, detailed numerical simulations must be performed, and these are in progress (Lípari et al. 2000b). The first results of these kinds of simulations suggest that the two massive galaxies are the main component of the merger event and that the satellite generally merges later (Y. Taniguchi 1999, private communication). This type of composite model-major plus minor mergers - could give an explanation of the very similar properties (i.e., radio emission, mass, etc.) found between R1 and R3, and some differences observed between these main/ massive nuclei and the nucleus R2. Another interesting possibility is the simultaneous major merger of three massive and gas-rich Sc spirals; however, this type of model 
requires even more complex numerical simulations (J. Navarro 1999, private communication).

Finally, the presence of the merger/interacting system NGC $3256 \mathrm{C}$ very close to NGC 3256 plus the close presence of NGC 3263 group of interacting galaxies and the merger NGC 3256A (see § 3.4) suggest the possibility of mergers between mergers. Additionally, this fact reinforces the idea that multiple mergers (in ULIRGs and LIRGs), in a scale of increasing global mass, will likely generate elliptical and even cD or radio galaxies (Schweizer 1982, 1990; Graham et al. 1984; Sanders et al. 1988a; Lípari et al. 1994; Taniguchi \& Shioya 1998). More specifically, detailed photometric and kinematical studies of "typical" IR mergers (see Shier, Rieke, \& Rieke 1994, 1996 and Shier \& Fischer 1998 for references) show that "standard" mergers could generate mainly low-luminosity ellipticals; and some ULIRGs could produce high-luminosity and massive ellipticals. Our results - for NGC 3256 - suggest that (1) the multiple merger process combined with the induced extreme starburst/galactic wind (see § 4.5) are two main physical processes that need to be considered in order to explain extreme properties in some LIRGs/ULIRGs; and (2) the multiple merger process is a natural way to explain the formation of some massive ellipticals from LIRGs/ ULIRGs, especially in compact groups and at the center of clusters of galaxies.

\subsection{The Nature of the Asymmetrical Spiral Arms}

The three asymmetrical spirals arms show all "very similar physical conditions," i.e., high-metallicity (low-ionization) giant $\mathrm{H}$ II region properties, with temperature of the ionizing sources $T_{\text {eff }} \sim 35,000 \mathrm{~K}$ and solar abundance, out to $r \sim 5 \mathrm{kpc}$. In addition, the two optical nuclei show also similar physical properties (see $\S \S 3.1$ and 3.3).

The three asymmetrical arms have one-arm structure (although arm I has a small counterarm, its length is much shorter). In merger processes, such asymmetrical-or onearm - spiral structures of young star formation and $\mathrm{H}$ II regions were studied by Mihos \& Hernquist (1996) and Taniguchi \& Wada (1996). These arms may be driven mainly by (case I) the triggering of a young stellar component by the dynamical effect of disk/bulge/halo major merger process (Mihos \& Hernquist 1996: see their Figs. 4 and 2), where the inflow phase plays a main role; or (case II) the triggering of star formation by the effect of highly asymmetrical gravitational potential, mainly in minor mergers (Taniguchi \& Wada 1996: see their Fig. 7, where the model $\mathrm{B}^{+}$- for prograde orbit - shows very similar properties to those detected in the arms and nuclear disks in NGC 3256).

The highly asymmetrical gravitational potential (case II), arises in (1) binary (or multiple) black hole in a galactic nucleus, (2) two (or multiple) nuclear star clusters in a galactic nucleus, (3) nuclear star cluster plus a black hole in a galactic nucleus, (4) pair (or multiple) extragalactic nuclei; and when the masses are significantly different by a factor 2 or more (Taniguchi \& Wada 1996). For NGC 3256 we found $(\S 3.1)$ an elongated main structure in the core of R1 (plus a compact knot) and two compact knots (of different size) in the core of R2. In addition, both regions have spectra with only nuclear starburst features. Therefore, the presence - in both nuclei - of two nuclear star clusters (or a bipolar elongated star cluster, or a star cluster plus a lowmass nuclear black hole) could be a likely option to consider for the origin of the asymmetrical spiral arm structures, as observed in NGC 3256. However, in the next section we consider that the processes of large infall of matter plus outflow-in the ISM - are also important components for the origin of these extended spiral arms and massive star formation (i.e., not only dynamical effects).

On the other hand, the broadband $B V I$ photometry (see Table 3) shows similar colors in the three asymmetrical spiral arms, and the three nuclei. The arms show $B-V$ color in the range $\sim 0.5-0.8$ (Table 3), and all show-at least - one strong blue knot. These properties were also observed in the shape of the spectral continuum in each individual region (Fig. 3). However, the presence of dust made these similar photometric properties less clear, specially in arm 3. The optical $B-V$ colors observed in the arms and the three nuclei are compatible with evolutionary models of star clusters of $10-15 \times 10^{6} \mathrm{yr}$ (Garcia-Vargas 1991: their Table 2.7 and Fig. 2.23), for a mass of the star cluster of $M_{T}=3 \times 10^{8} M_{\odot}$.

Morphological, spectroscopic, and photometric data show similar properties in the arms and their nuclei (§ 3.1.1). These are new results that probably require a detailed theoretical study. However, a first and simple explanation is to consider that the physical conditions for the star formation process - in the central region - are similar at a scale of $r \sim 5-6 \mathrm{kpc}$. The galactic wind, detected in the central and nuclear regions of NGC 3256 (\$ 3.2.2 and Scarrott el al. 1996), could be the natural process in young starbursts (with an IMF that results in a high number of massive stars, an $\mathrm{SN}$ event, and the associated stellar wind; Robert et al. 1993) that generated similar conditions, at scale of $\sim 5-6$ kpc. We discuss in $\S \S 4.4$ and 4.5 a new scenario of nuclear star formation process in which the the relation between the galactic wind and the infalling gas plays a main role.

\subsection{The Complex Kinematics and Inflow in NGC 3256 (Ionized and Molecular Gas)}

Most of the features of the $\mathrm{H} \alpha \mathrm{VF}$ could be reproduced by the superposition of three rotation curves extracted at three very different position angles. They show the typical "sinusoidal" shape of merger galaxies (see $\S$ 3.2.1). We found that these RCs are defined mainly by the presence of outer noncircular motions, since the decrease in the external parts is steeper/higher than the values expected by a Keplerian velocity decay. This seems to be also the case for sinusoidal RCs observed in similar mergers: e.g., NGC 7252, 520, and 6240 (Schweizer 1982; Stanford \& Balcells 1990; Bland-Hawthorn, Wilson, \& Tully 1991; Zepf 1993). Therefore, this shape in the RCs is not directly related to the evolution of the halos and the "dark matter" of the original galaxies that collided.

An interesting test was performed using the molecular gas $\left({ }^{12} \mathrm{CO} J=2-1\right)$ velocity map, published by Aalto et al. (1991, their Fig. 6). First, we digitized this CO VF. This CO data have a resolution of $\sim 5^{\prime \prime}-7^{\prime \prime}$, and therefore in order to compare this $\mathrm{CO}$ map to our $\mathrm{H} \alpha$ data, we degraded our velocity field (using a Gaussian filter) to match the $\mathrm{CO}$ resolution. The result was a smooth $\mathrm{H} \alpha$ velocity field very similar to that obtained by Aalto et al. for CO (i.e., in each positive and negative part of the VF, the two/three subregions were almost combined in one extended lobe). Therefore, the lack of resolution in distant mergers could lead us to consider the noncircular motion as global rotation. In addition, we extracted the RCs from the digitized $\mathrm{CO} V \mathrm{VF}$ and the "smooth" $\mathrm{H} \alpha$ velocity fields (for a P.A. $\sim 90^{\circ}$ and 
for an aperture sector of $40^{\circ}$ ). And, we found highamplitude residuals corresponding to each of the "sinusoidal/decreasing RCs," indicating that the smoothed VF does not represent a real general motion.

For the circumnuclear region, $r \leq 10^{\prime \prime} \sim 1.8 \mathrm{kpc}$ (with a general circular motion), we derive a Keplerian mass of $M_{\text {c.nuclear }}=1.6 \pm 0.5 \times 10^{10} M_{\odot}$. Since the $A_{V} \sim 35$ and $L_{\mathrm{IR}} / L_{B} \sim 50$ (Lutz et al. 1996), we used the IR luminosity to derive a mass/luminosity ratio of $M_{\text {c.nuclear }} / L_{\mathrm{IR}} \sim 0.05$ for $r \sim 1.8 \mathrm{kpc}$. Previously, Feast \& Robertson (1978) obtained a lower limit for the total mass of $\sim 5 \times 10^{10} M_{\odot}$ and $M_{\text {total }} / L_{B} \sim 0.3$. In addition, Wright et al. (1988) deduced a $M_{\text {nucl.starburst }} \sim 7 \times 10^{8} \quad M_{\odot}$ and $M_{\text {nucl.starburst }} / L_{\mathrm{IR}} \sim 0.01$ (from the kinematical values obtained by Feast \& Robertson 1978). These results show a good agreement and also a good agreement with those obtained for major mergers between gas-rich Sc spirals and mergers with strong nuclear massive star formation processes (see Wright et al. 1988).

An interesting kinematic result, presented in $\S 3.2$, is the detection of inflow in a $r \sim 4^{\prime \prime} \sim 700 \mathrm{pc}$, close to the nuclear region (i.e., around the main optical nucleus, which is located near to the kinematical center at $\sim 400 \mathrm{pc}$ ). In particular, we found - for NGC 3256 - kinematic and morphological evidences supporting the nuclear inflow scenario, and these results are in agreement with the predictions of numerical models for major and minor mergers: "where starbursts are driven by merger processes by depositing large amount of interstellar gas to the nuclear region" (see Mihos \& Hernquist 1996, 1994a, 1994b; Taniguchi \& Wada 1996; Barnes \& Hernquist 1992).

The detection of the superposed sinusoidal curves in the nuclear region $\left(r \leq \sim 700\right.$ pc) for a range of P.A. $40^{\circ}-130^{\circ}$ could be generated mainly by the presence of nuclear inflow or outflow (Lípari et al. 1993b). However, this range $\left(40^{\circ}-\right.$ $130^{\circ}$ ) of P.A.'s include the P.A. range in which we found an absence of the nuclear outflow component $\left(45^{\circ}-80^{\circ}\right)$, and therefore, the more likely explanation for this shape in the $\mathrm{RCs}$ is the presence of nuclear inflow. This is also in agreement with the observed near/far side orientation $(\S 3.2)$. Furthermore, a very interesting fact is that the mean value of the P.A. - in the inflow region (at P.A. $\sim 80^{\circ}$ ) is practically perpendicular to the axis of the bipolar outflow (at P.A. $150^{\circ}-160^{\circ}$ ), which is predicted by models of outflow generated in nuclear star clusters (Perry 1992), and it is expected if the inflow material (ionized and molecular gas) contributes to the collimation process of the outflow material (Suchkov et al. 1996).

It is interesting to note that in Arp 220, all the "luminous blue star cluster (LBSCs)" (Shaya et al. 1994) are mainly located in the direction perpendicular to the bipolar outflow's axis (where the interaction between the outflow and inflow material is probably generated). And we suggest, in $\S \S 4.4$ and 4.5 , that in NGC 3256 the nuclear blue star cluster was generated mainly by the interaction of the inflow and outflow processes. However, the situation in NGC 3256 is more complex, since the young globular cluster candidates are mainly located in the nuclear and in the three asymmetrical spiral arms (see Zepf et al. 1999, $\S 3.1 .1$, and Figs. $1 d$ and $1 e$ ). We discuss this point in $\S 4.4$.

If we assume that the inflow rate is moderate/low (see Heller \& Shlosman 1994 for references), we can estimate the mass of the main nucleus, for a $r \leq 3^{\prime \prime} \sim 500$ pc where we detected a maximum in the inflow curve $\left(\Delta V_{\max } \sim 65 \mathrm{~km}\right.$ $\mathrm{s}^{-1}$ ). Using a spherical potential model (Bertola et al. 1991; Lípari et al. 1993b) and assuming that the inflow motion is close to circular, we derived a mass of $M_{\text {region } 1} \sim 0.5 \times 10^{9}$ $M_{\odot}$. For region 3, a similar value of the mass could be assumed, according to the result obtained in $\S 3.2 .1$ (the kinematical center is located midway between both nuclei). These values of mass are in good agreement with those expected for galactic nuclei with "massive" stars clusters: $\sim 0.3 \times 10^{9} M_{\odot}$ (Garcia Vargas 1991, and see $\left.\S 4.2\right)$ and also are similar to the deduced value of the nuclear starburst mass $\sim 0.7 \times 10^{9} M_{\odot}$ (Wright et al. 1988).

It is important to note that all detailed evolutionary nuclear starburst models (see Tenorio-Tagle \& MuñozTuñon 1997) require the presence of large infall of matter to the central and nuclear regions (at $\sim \mathrm{kpc}$ scale) as an indispensable condition in order to obtain a large SFR and starburst for $\sim 10^{7} \mathrm{yr}$ (as in NGC 3256 and LIRG/ULIRGs). These infalling materials could change strongly the conditions in the ISM and therefore could play an important role in the evolution of the star formation rate (SFR) and the galactic winds.

The scale of the inflow detected in NGC $3256(r \sim 500 \mathrm{pc})$ also required the study of models of small-scale inflow ( $\sim 10^{2}$ pc). Specifically, Perry (1992) and Williams \& Perry (1994) found very interesting results modeling the outflow of a nuclear stellar cluster and the flow in the ISM (using two-dimensional hydrodynamical, evolutionary population synthesis, and stellar evolutionary track models). They found for the high mass-loss stage (of the stellar cluster) the following: (1) a super/hypersonic outflow with wide opening angle (close to spherical geometry); (2) half of the outflow mass of the cluster is accreted; (3) this infall is roughly perpendicular to the axis of the outflow; (4) a peak luminosity $L_{\text {bol }} \sim 10^{47} \mathrm{ergs} \mathrm{s}^{-1}$ requires an initial cluster stellar mass of $M_{\text {cluster }} \sim 2 \times 10^{9} M_{\odot}$ and initial average stellar density of $\sim 4 \times 10^{8} M_{\odot} \mathrm{pc}^{-3}$. The observed properties of the nuclear inflow detected in NGC 3256 show a good agreement with these theoretical results.

\subsection{The Star Formation in NGC 3256 and LIRGs/ULIRGs}

The new results presented in $\S 3$ are clearly compatible with an extended massive star formation plus a powerful galactic wind, driven by the merger process, and this extended massive star formation process is the main source of high IR luminosity observed in this galaxy. Similar results were found for practically all the prototypes of LIRGs and ULIRGs (see Lutz et al. 1996; Genzel et al. 1988; Downes \& Solomon 1998), where the evidences of the starburst and galactic wind components are frequently less evident than in the nearby merger NGC 3256. In Arp 220, Smith et al. (1998) detected the presence of multiple radio $\mathrm{SNe}$ in the nuclear regions, and they also found that the IR properties could be explained mainly by the presence of a strong starburst. Previously, Rieke et al. (1985), Heckman et al. (1987), and Lípari et al. (1994, 1993a) suggested a similar starburst/galactic wind scenario for the luminous IR mergers Mrk 231, Arp 220, and NGC 6240, where the AGN component is probably strongly obscured or is not active. Recently, new ISO and CO observations of luminous IR mergers confirm that the starburst is mainly the dominant source of IR luminosity in these systems (Genzel et al. 1998; and Downes \& Solomon 1998).

The optical photometric, kinematic, and spectroscopic results $(\S 3)$ complete - together with those obtained pre- 
viously (specially the data obtained by Calzetti et al. 1994; Storchi-Bergmann et al. 1995, where a study of multiwavelenth spectra was also performed) - an interesting multiwavelength set of data for NGC 3256. Previously, only for few starburst galaxies (e.g., NGC 7714) was it possible to make this type of multiwavelength study of star formation. Using this new set of data, we found interesting properties in NGC 3256:

1. At optical wavelengths, the $\mathrm{EW}(\mathrm{H} \alpha+[\mathrm{N} \mathrm{II}])$ for the region $1+2$ is $\sim 430 \AA$ (Table 4). This value is one of the highest known for mergers (see Fig. 2 by Liu \& Kennicutt 1995: $\mathrm{EW}(\mathrm{H} \alpha+[\mathrm{N} \mathrm{II}])$ for the central region of 40 mergers), and this EW parameter is considered as a direct index of SFR in starburst galaxies.

2. At UV wavelengths, the measured values of $E W$ and blueshift in the two main absorption lines $V_{\mathrm{Si} \mathrm{IV}}=-750$ $\pm 60 \mathrm{~km} \mathrm{~s}^{-1}$ and $V_{\mathrm{C}_{\text {IV }}}=-1070 \pm 40 \mathrm{~km} \mathrm{~s}^{-1}$ (Table 6) show a very interesting agreement with the predictions of synthetic models of starburst with galactic wind from massive stars. Specifically, our values are in agreement with models of a instantaneous burst of $10 \times 10^{6} \mathrm{yr}, Z=Z_{\odot}$, and $M_{u}=$ $60 M_{\odot}$ (Robert et al. 1993, their Fig. 4).

3. Using the optical and near-IR emission-line ratios of [S II] $\lambda \lambda 6717+6731 /[\mathrm{S}$ III $] \lambda \lambda 9069+9532$ (for region $1+2$; Table 4) and the correlation between $\log (u)$ and [S II]/ [S III] (from Diaz et al. 1991 and Garcia-Vargas 1991), we found a deduced value of the ionization parameter $\log (u)=-3.0$. This is in good agreement with the value obtained in $\S 3.3$ (using the diagrams of Pastoriza et al. 1993; and for each individual regions).

4. At near-IR wavelengths, the absorption line of the $\mathrm{Ca}$ II triplet shows for the two prominent lines EW $(\lambda \lambda 8542+8662) \sim 8.8 \AA$ (Table 6$)$, which is a normal value for starburst galaxies (see Terlevich, Diaz, \& Terlevich 1990, their Fig. 5). This value is compatible with evolutionary models of star clusters of $4-15 \times 10^{6} \mathrm{yr}$ (Garcia-Vargas 1991, their Table 4.1 and Fig. 4.2) for standard parameters in the IMF. Therefore the range of age derived in this work for the extended massive star formation detected in NGC 3256 is $\sim 5-25 \times 10^{6} \mathrm{yr}$ (and the more probable value is $\sim 10 \times 10^{6} \mathrm{yr}$ ).

An interesting point is the difference found, using ISO IR data, between the properties of the starburst and the massive star formation (i.e., $T_{\text {eff }}$ and the upper mass limit for the ionizing sources) obtained by Rigopoulou et al. (1996) and our results ( $(3.3 .3)$. A first simple idea is that the use of optical observations could lead to biased low values for the high-mass cutoff, since the most massive stars are not visible at optical wavelengths (Mirabel et al. 1998). However, for NGC 3256, Joseph (1991), Doyon et al. (1994), and Moorwood \& Oliva (1994) using near-IR data obtained similar results to those presented in $\S$ 3.3.3. At least the differences in the $T_{\text {eff }}$ values of the ionizing sources could be easily explained by the fact that the $T_{\text {eff }}$ obtained by Rigopoulou et al. (1996) is $\sim 41,000 \pm 3000 \mathrm{~K}$, but Lutz et al. (1996) suggested that using better stellar atmosphere models than Kurucz (1992), the $T_{\text {eff }}$ decreases in $\sim 2000 \mathrm{~K}$. Recently, there was also important improvement in modeling the ionizing source of $\mathrm{H}$ II regions, using mainly the continuum from star clusters and not merely from one type of early $O B$ stars (Garcia-Vargas et al. 1997, 1995a, 1995b; Pastoriza et al. 1993). Therefore, our value of $T_{\text {eff }}=35,000 \pm 3000 \mathrm{~K}$ is the same - within the errors - to that obtained by Rigopou- lou et al. (1996). On the other hand, the results of the upper mass limit for the ionizing sources-which are closely related to the $T_{\text {eff }}$-are model dependent (Garcia-Vargas et al. 1997, 1995a, 1995b), and therefore, their discussion required a specific study of the IMF, the star formation histories, and the starburst process (i.e., constant SFR, $\delta$ burst, extended burst, etc.; see Lípari et al. 2000b). However, our UV-IUE data combined with synthetic starburst/stellar wind models show a good agreement with a upper mass limit of $\sim 60 M_{\odot}$ (which is the same value obtained by Rigopoulou et al. 1996).

Finally, an important result-obtained in $\S 3.1$-is the detection at the core of the main optical nucleus (R1) of a blue elongated structure: a disk of $50 \mathrm{pc} \times 25 \mathrm{pc}$, which in the color map (Fig. $1 f$ ) shows inside an interesting morphology: a compact blue knot of $r \sim 8$ pc (probably a luminous blue stellar cluster) and two extended/weak blue regions. The elongated structure of R1, with disk morphology (disk within disk), could be associated with the nuclear starburst process, and Feldman et al. (1982) suggestedfrom dynamical arguments - this type of disk structure for nuclear starbursts cores. In addition, we detected in R1 and R2 (§ 3.1.1) several other blue compact knots, which could be also associated with young/blue stellar clusters (with $r \sim 8-10 \mathrm{pc}$ ).

Recently, Zepf et al. (1999) published a detailed HST study of candidate young globular clusters in NGC 3256, and they found more than 1000 compact blue objects mainly distributed in the nuclei and the central regions. These results are in good agreement with the extended massive star formation process proposed in for this merger. However, it is important to note that high-resolution HST observations of nearby mergers show different results related to the population, sizes,and types of young/blue stellar clusters (see, for references, Whitmore et al. 1999), and this fact is probably related to the dynamical and physical conditions in each particular merger. In particular, in Arp 220 at the core of the main/west nucleus, an elongated stellar association (of $75 \mathrm{pc}$ ) with a luminous blue star cluster was found by Shaya et al. (1994), using HST broadband images. Therefore, the very nuclei of NGC 3256 and Arp 220 show very similar properties, and Taniguchi et al. (1998) suggested that this particular type of "superluminous blue star clusters" (found mainly at the cores of ULIRGs) is formed when the nuclear infalling dense gas collapses to form massive gaseous clumps, and then massive star formation is induced in them by the effect of the external pressure that is driven by the galactic wind. We detected, in NGC 3256, all the components required for the formation of LBSCs (in particular, inflow plus galactic wind). In the next section we also discuss other properties of the extended massive star formation process.

\subsection{The Galactic Wind in NGC 3256 and LIRGs/ULIRGs}

Graham et al. (1984), HAM90, and Scarrott et al. (1996) already suggested - for NGC 3256 - extended outflow and galactic winds originated in a strong starburst. However, the results of the present work (\$ 3.2.2) are the first direct kinematic evidence for outflow, directly associated with the nuclear starburst: i.e., "galactic winds." Since, there is not evidence of $A G N$ properties in all the multiwavelength studies of NGC 3256 (see, for details, Kotilainen et al. 1995).

In $\S 3.2$, we found that the "nuclear" outflow in NGC 3256 shows $V_{\text {nuc.outflow }} \sim-350 \mathrm{~km} \mathrm{~s}^{-1}$, FWHM $\sim 130 \mathrm{~km}$ $\mathrm{s}^{-1}$, bipolar structure (i.e., without component at P.A. $45^{\circ}-$ 
$\left.80^{\circ}\right)$, and a "very" wide opening angle $\left(\theta \sim 140^{\circ}\right)$. In addition, we detected that this blue component is extended in a $r \sim 5-6 \mathrm{kpc}$. These results are in agreement with the prediction made for the blowout phase of the galactic wind after $8 \times 10^{6} \mathrm{yr}$ from the initial burst, i.e., in the beginning of the dusty starburst phase of Type II SNe (Lípari et al. 1994; Norman \& Ikeuchi 1989; Terlevich et al. 1992). Furthermore, the detection of "radial filaments in practically all the outer regions" of NGC 3256 (see $\S 3.1 .2$ and Fig. $1 b$ ) plus the age of the massive star formation process are also in agreement with the blowout phase of the galactic wind. The polarimetry study of NGC 3256 also shows the typical "extended radial field," similar to those found in galaxies in the blowout phase of the galactic wind (Scarrott et al. 1996).

In addition, we measured for P.A. $110^{\circ}$ the blue and red components in the nuclear outflow: $V_{\mathrm{OF}-\text { blue }}=-350 \pm 30$ $\mathrm{km} \mathrm{s}^{-1}$ and $V_{\text {OF-red }}=+350 \pm 50 \mathrm{~km} \mathrm{~s}^{-1}$. In addition, Figure $2 f$ shows - for PA $18^{\circ}$ - similar values in the blue and red outflow velocity in the external regions. Assuming the biconic surface model for the main origin of the outflow emission lines (see, for references, HAM90 and Colina et al. 1991), similar values of both outflow components suggest that the angle between the bicone axis and the line of sight $\left(i_{c}\right)$ is close to $90^{\circ}$, and therefore using the value of the opening angle of the cone $(\theta)$ we found a value for the velocity of the outflow $V_{\text {outflow }}=370 \mathrm{~km} \mathrm{~s}^{-1}\left(V_{\text {OF-blue }}=\right.$ $\left.-V_{\text {outflow }} \times \cos \left[i_{c}-\theta / 2\right]\right)$. In this simplest model the $V_{\text {outflow }}, \theta$, and $i_{c}$ are all constant and independent of the distance from the nucleus.

Two complex and related aspects, in the theoretical studies of the galactic wind, are the modeling of the collimation process and the evolution of the geometry. The collimation process was studied mainly for spiral galaxies, and for the evolution of the geometry (which appears intimately connected with the structure of the ISM), two main ideas were proposed: (1) bipolar galactic wind arising in the plane-parallel gaseous atmosphere of a galactic disk (e.g., Tomisaka \& Ikeuchi 1988; Suchkov et al. 1994, 1996), which is important in the initial phases; and (2) spherical symmetric galactic wind for the late phases (e.g., Mathews \& Dones 1992; Chevalier \& Clegg 1985), where the wind will lose memory of the initial geometry. Our results for NGC 3256 show composite structure: bipolar outflow (with symmetry axis at the P.A. $\sim 150^{\circ}$ ), but with a very wide opening angle $\left(\theta \sim 140^{\circ}\right)$, which is practically a spherical geometry. This opening angle is higher than the mean values found in the sample of HAM90: $\sim 60^{\circ}-80^{\circ}$, but this range was found mainly for spiral galaxies with galactic wind (NGC 253, M82, and NGC 4945). However, our previous results for the "super-antennae" (another IR merger) also show very wide opening angle in the outflow component: $\theta \sim 100^{\circ}$ (Colina et al. 1991). Therefore, the properties of the galactic wind in mergers show clear differences with those found in spiral galaxies.

In addition, we note that the orientation of the outflow bipolar axis is located close to the orientation of the photometric and the main kinematic minor axis of the merger: P.A. $\sim 170^{\circ}-160^{\circ}$ and P.A. $\sim 00^{\circ}-170^{\circ}$, respectively (the P.A. of the photometric minor axis was obtained fitting ellipses to the ESO NTT $V$ broadband image). Previously, Moorwood \& Oliva (1994) found a P.A. of $\sim 162^{\circ}$ for the near-IR photometric minor axis, fitting ellipses to the $K^{\prime}$ image, and they show that over the central $15 \mathrm{kpc}$, the $K^{\prime}$ image is not yet that of a relaxed system $\left(r^{1 / 4}\right.$ profile $)$.
An important point to consider is that the outflow component shows high emission-line ratios $[\mathrm{N} \mathrm{II}] \lambda 6584 / \mathrm{H} \alpha$ and [S II] $\lambda \lambda 6717+6731 / \mathrm{H} \alpha(\S 3.2 .2)$. We found a similar result for the outflow component in the nearby starburst and Seyfert nucleus of NGC 4945 (Lípari et al. 1997), and we suggested that this interesting result could be explained by a high abundance of $\mathrm{N}$ generated by the presence of massive stars (especially, the early WN type of Wolf-Rayet stars) plus shock processes. In addition, we found (§ 3.3.2) that also the "main component" of the emission lines in the external regions with outflow shows very high values in these two emission-line ratios.

Using the emission-line ratios obtained for the nuclear outflow component (\$ 3.2.2) and for the external regions in the main emission-line component (§ 3.3.2) plus the diagnostic diagram of Veilleux \& Osterbrock (1987) and HAM90 (Fig. 14), we found that these values are inconsistent with gas heating by $\mathrm{H}$ II regions (where $[\mathrm{N} \mathrm{II}] \lambda 6584 / \mathrm{H} \alpha \leq 0.45$, and [S II] $\lambda \lambda 6717+31 / \mathrm{H} \alpha \leq 0.32$, for $\mathrm{H}$ II regions). These ratios are clearly consistent with shocks driven into clouds accelerated outward by a starburst with galactic wind: these values - for the outflow and main components - are located in the diagnostic diagram of HAM90 (their Fig. 14) in the region of SN remnants, shock heating, and Herbig-Haro objects ([S II $] \lambda 6717+31 / \mathrm{H} \alpha \geq 0.32$; see Lípari et al. 1997).

On the other hand, at X-ray wavelengths, NGC 3256 is the only H II galaxy in the Boller et al. (1992) complete sample catalog of IRAS and ROSAT sources confirmed as an luminous X-ray and IR emitter by Moran, Halpern, \& Helfand $(1994,1996)$ : with $L_{\mathrm{X}} \geq 10^{42} \mathrm{ergs} \mathrm{s}^{-1}$. According to the main results presented in $\S 3$, i.e., the presence of an extended starburst with galactic winds, the sources of the high X-ray emission could be attributed mainly to SN remnant plus emission from the hot phase of the ISM associated with the galactic winds (Suchkov et al. 1994). Furthermore, we measure a value of $\log \left(L_{\mathrm{X}} / L_{\mathrm{IR}}\right)=-2.8$, which is a typical value for a starburst galaxy with galactic wind: e.g., M82, NGC 253, NGC 3079, Arp 220, NGC 3628, and NGC 3690 (see HAM90, their Table 7).

The simultaneous presence of "galactic wind" and "inflow" (in the main optical nucleus of NGC 3256) could represent observational evidence that supports the new models of nuclear star formation suggested by Taniguchi et al. (1998) and Shlosman \& Noguchi (1993); see § 4.4. Furthermore, the galactic wind could be a natural process of a starburst driven by a starburst, in a cloudy protogalaxy that evolves by multiple mergers (Berman \& Suchkov 1991; Suchkov \& Berman 1992; Marochnik \& Suchkov 1992). In this scenario the strong galactic wind driven by the initial burst of star formation plays an important role in the next step of the merger evolution; in particular, for new star formation processes. It is important to note that exactly in the same region where we detected the galactic wind $(r \sim 5-$ $6 \mathrm{kpc}$ ), previously Sargent et al. (1989) reported extended molecular $\mathrm{H}_{2}$ gas (for $r \sim 6 \mathrm{kpc}$ ), and these are the main components in the extended star formation scenario proposed in this work.

In addition, in order to study in detail the main and the feedback processes that produce the extended massive star formation, it is also important to consider another component (especially in systems with strong starburst and galactic wind): the low-energy cosmic rays, which-together with the shock process associated with the galactic windplay an important role as strong sources of heating and 
pressure of molecular clouds (Allen 1992; Suchkov, Allen, \& Heckman 1993). These cosmic rays are probably produced at a rate proportional to the $\mathrm{SN}$ rate and could be evacuated by galactic wind (Suchkov et al. 1993). Additionally, studies of nuclear starburst/galactic wind in IR mergers and spiral galaxies have provided evidence for extremely high pressures in these regions: 3-4 orders of magnitude higher than typical pressure in the ISM in our Galaxy (Schaaf et al. 1989; HAM90). Therefore, the contribution of cosmic rays could help to explain the high-pressure environment and the warm temperatures observed in the ISM of these systems (and specially in the molecular clouds). These type of physical conditions are required by our proposed scenario for the formation of extended massive star formation in NGC 3256 (according to the general scenario suggested by Shlosman \& Noguchi 1993 and Taniguchi et al. 1998).

Consequently, probably the interaction between dynamical effects, the galactic wind (outflow), low-energy cosmic rays, and the molecular + ionized gas (in the inflow phase) could be the possible mechanism that generates the "similar extended properties in the massive star formation, at scale of 5-6 kpc."

\section{CONCLUSIONS}

This work shows that understanding the complex scenario of merger, starbutst, and galactic wind processes at low and high redshift strongly depends on resolution and the quality of the data, and even for NGC 3256 (the nearest advanced IR merger with starburst and galactic wind) only a complete and detailed set of multiwavelength observations obtained using high and moderate spatial resolution shows "clean" evidence of galactic wind, nuclear starburst/inflow, and extended massive star formation (which are superposed to merger features). In particular, the analysis of the "real morphology" of the extended massive star formation required ESO NTT-SUSI observations with a spatial resolution better than 0".7 (and even the ESO NTT-EMMI data-obtained with 1"4 resolution-shows mainly confused structures, which look like partial rings), and the "outflow and inflow component" detection required at least spectra obtained with moderate/high resolution (better than $100 \mathrm{~km} \mathrm{~s}^{-1}$; see $\S 3.2$ ) and covering a complete set of position angles (since the outflow and inflow components are weak or absent at the P.A. intervals $45^{\circ}-80^{\circ}$, and $0^{\circ}-$ $40^{\circ} / 130^{\circ}-180^{\circ}$, respectively). We also found that the kinematic presence of "sinusoidal RCs (in mergers)" is mainly indicative of noncircular motion (and is not a direct evidence of merger process; Zepf 1993). Therefore, in order to obtain clear evidence of starburst, galactic wind, and mergers in distant galaxies/QSOs, a detailed and complete multiwavelength set of high-quality data is required (including images, spectrophotometry, and kinematics), added to a careful consideration of different theoretical interpretations.

In conclusion, the results obtained in this paper suggest that NGC 3256 is the product of a multiple merger, which generated an extended massive star formation process with an associated galactic wind plus a nuclear inflow. In particular, we found for the merger NGC 3256 (and previously in Mrk 231, IRAS 19254-7245/super-antennae, and probably in IRAS $07598+6508$ ) detailed kinematic, physical, and morphological evidence that "young starbursts" in luminous IR mergers show the typical features of galactic wind. In addition, we detected in IRAS 22419-6049 the first type 1 IR QSO that shows Wolf-Rayet and interacting features (Lípari \& Macchetto 1992; previously Armus, Heckman, \& Miley 1988 found Wolf-Rayet features also in an IR QSO of type 2). Therefore, these and previous results (see HAM90, where $70 \%$ of their sample of galaxies showing galactic winds are mergers!) strongly suggest that the relation between merger and starburst (with galactic wind and "multiple" Type II SN explosions) plays a main role in the evolution and formation of galaxies (see Lípari et al. 2000a).

The authors wish to thank V. Afanasiev, J. Ahumada, J. Boulesteix, A. Diaz, D. Garcia-Lambas, J. Melnick, A. Micol, A. Moorwood, J. Navarro, M. Nicotra, S. d'Odorico, M. Pastoriza, T. Storchi-Bermann, A. Suchkov, E. Terlevich, K. Thompson, and S. Zepf for discussions and help. We would like to express our gratitude to the staff members and observing assistants at BALEGRE, CASLEO, CTIO, and ESO Observatories. This work was based on observations made using the NASA and ESA $H S T$ and $I U E$ satellites, obtained from archive data at ESO-Garching and STScI-Baltimore. This work was made using the NASA/ IPAC Extragalactic Database (NED), which is operated by the Jet Propulsion Laboratory, California Institute of Technology, under contract with NASA. S. L. was supported in part by Grants from Conicet, Conicor, Secyt-UNC, Fundación Antorchas (Argentina), and STScI (USA). R. D. acknowledges a fellowship from FOMEC (FaMAF) at Cordoba University. Finally, we wish to thank the referees for valuable and constructive comments.

\section{REFERENCES}

Aalto, S., et al. 1991, A\&A, 247, 291

Afanasiev, V., Dodonov, S., \& Carranza, G. 1994, Bol. Assoc. Argentina Astron., 39, 160

Aguero, E., \& Lípari, S. L. 1991, Ap\&SS, 75, 253

Allen, R. 1992, ApJ, 399, 573

Aller, L. 1984, Physics of Thermal Gaseous Nebulae (Dordrecht: Reidel)

Armus, L., Heckman, T. M., \& Miley, G. 1988, ApJ, 326, L45

Barnes, J. 1989, Nature, 338, 123

. 1992, ApJ, 393, 484

Barnes, J., \& Hernquist, L. 1992, ARA\&A, 30, 705

.1996, ApJ, 471, 115

Berman, B., \& Suchkov, A. 1991, Ap\&SS, 184, 169

Bertola, F., et al. 1991, ApJ, 373, 369

Bevington, P. 1969, Data Reduction and Error Analysis for the Physical

Sciences (New York: McGraw-Hill)

Bland, J., Taylor, K., \& Atherton, P. 1987, MNRAS, 228, 591

Bland, J., \& Tully, R. 1988, Nature, 334, 43

Bland-Hawthorn, J., Wilson, A., \& Tully R. 1991, ApJ, 371, L19

Boller, T., et al. 1992, A\&A, 261, 57
Calzetti, D., Kinney, A., \& Storchi-Bergmann, T. 1994, ApJ, 429, 582

Carral, P., et al. 1994, ApJ, 423, 223

Chevalier, R., \& Clegg, A. 1985, Nature, 317, 44

Clements, D., et al. 1996, MNRAS, 279, 459

Colina, L., Lípari, S. L., \& Macchetto, F. 1991, ApJ, 379, 113

Condon, J., Anderson, M., \& Helou, G. 1991a, ApJ, 376, 95

Condon, J., Huang, Z., Yin, Q. \& Thuan, T. 1991b, ApJ, 378, 65

de Vaucouleurs, G., \& de Vaucouleurs, A. 1961, MmRAS, 68, 69

Diaz, A., et al. 1987, MNRAS, 226, 19 1991, MNRAS, 253, 245

Diaz, A., \& Perez-Montero, E. 1998, ESO Workshop, in press 1999, MNRAS, submitted

Diaz, R., Carranza, G., Dottori, H., \& Goldes, G. 1999, ApJ, 512, 623

Djorgovski, S. 1994, in Proc. Conf. Mass-Transfer Induced Activity in

Galaxies, ed. I. Shlosman (Cambridge: Cambridge Univ. Press), 452

Dopita, M., \& Evans, G. 1986, ApJ, 307, 431

Downes, D., \& Solomon, P. M. 1998, ApJ, 507, 615

Doyon, R., Joseph, R. D., \& Wright, G. S. 1994, ApJ, 421, 101

Fabbiano, G. 1988, ApJ, 330, 672 
Feast, M., \& Robertson, B. 1978, MNRAS, 185, 31

Feldman, F., Weedman, D., Balzano, V., \& Ramsey, L. 1982, ApJ, 256, 427

Ferland, G. 1991, Internal Report 91-1, Astronomy Dept., Ohio State University

Filippenko, A. \& Sargent, W. 1992, AJ, 103, 28

Fouque, P. 1992, A\&AS, 93, 211

Franco, J., \& Ferrara, A. 1992, in Evolution of ISM and Dynamics of Galaxies, ed. J. Palous, W. Burton, \& P. Lindbland (Cambridge: Cambridge Univ. Press), 130

Garcia, A. M. 1993, A\&AS, 100, 47

Garcia-Vargas, M. 1991, Ph.D. thesis, Univ. Autonoma de Madrid, Spain

Garcia-Vargas, M., Bressan, A., \& Diaz, A. 1995a, A\&AS, 112, 13 1995b, A\&AS, 112, 35

Garcia-Vargas, M., \& Diaz, A. 1994, ApJS, 91, 553

Garcia-Vargas, M., et al. 1997, ApJ, 478, 112

Genzel, R., et al. 1998, ApJ, 498, 579

Graham, G., Wright, G., Meikle, W., Joseph, R., \& Bode, M. 1984, Nature, 310,213

Heckman, T. M., Armus, L., \& Miley, G. 1987, AJ, 93, 276 1990, ApJS, 74, 833 (HAM90)

Heller, C., \& Shlosman, I. 1994, ApJ, 424, 84

Hibbard, J. E., et al. 1994, AJ, 107, 67

Hutching, J. B., \& Neff, S. 1991, AJ, 101, 434

Joseph, R. D. 1991, in Massive Stars in Starbursts, ed. C. Leitherer,

N. Walborn, T. M. Heckman, \& C. A. Norman (Cambridge: Cambridge Univ. Press), 259

Joseph, R. D., \& Wright, G. S. 1985, MNRAS, 214, 87

Kennicutt, R., Keel, W., \& Blaha, C. 1989, AJ, 97, 1022

Kobulnicky, H., Skillman, E., Roy, J., Walsh, J., \& Rosa, M. 1997, ApJ, 477, 679

Kotilainen, J., Moorwood, A., Ward, M., \& Forbes, D. 1995, A\&A, 305, 107

Kobo, O., \& Sternberg, A. 1996, in preparation

Kurucz, R. 1992, Rev. Mexicana Astron. Astrofis., 23, 181

Landolt, A. U. 1992, AJ, 104, 340

Laubert, A et al. 1978, A\&AS, 35, 55

Leitherer, C. 1991, in Massive Stars in Starbursts, ed. C. Leitherer, N. Walborn, T. M. Heckman, \& C. A. Norman (Cambridge: Cambridge Univ. Press), 1

Leitherer, C., \& Heckman, T. 1995, ApJS, 96, 9

Lípari, S. L., Colina, L., \& Macchetto, F. 1994, ApJ, 427, 174

Lípari, S. L., et al. 2000a, in preparation

. 2000b, in preparation

Lípari, S. L., \& Macchetto, F. 1992, ApJ, 387, 522

Lípari, S. L., Terlevich, R., \& Macchetto, F. 1993a, ApJ, 406, 451

Lipari, S. L., Tsvetanov, Z., \& Macchetto, F. 1993b, ApJ, 405, 586 1997, ApJS, 111, 369

Liu, Ch., \& Kennicutt, R., Jr. 1995, ApJ, 450, 547

Lutz, D. 1991, A\&A, 245, 31

Lutz, D., et al. 1996, A\&A, 315, L137

Marochnik, L., \& Suchkov, A. 1992, in Evolution of ISM and Dynamics of Galaxies, ed. J. Palous, W. Burton, \& P. Lindbland (Cambridge: Cambridge Univ. Press), 234

Mathews, W. G., \& Dones J. 1992, Lick Obs. Bull., preprint

McCarthy, P., Heckman, T., \& van Breugel, W. 1987, AJ, 93, 264

McQuade, K., Calzetti, D., \& Kinney, A. 1995, ApJS, 97, 331

Melnick, J., \& Mirabel, I. F. 1990, A\&A, 231, L19

Mihos, C., \& Hernquist, L. 1994a, ApJ, 425, L13 1994b, ApJ, 431, L9 1996, ApJ, 464, 641

Mirabel, I. F., et al. 1998, A\&A, 333, L1

Mirabel, I. F., Dottori, H., \& Lutz, D. 1993, A\&A, 256, L19

Mirabel, I. F., Lutz, D., \& Maza, J. 1991, A\&A, 243, 367

Moorwood, A. F., \& Oliva, E. 1994, ApJ, 429, 602

Moran, E., Halpern, J., \& Helfand, D. 1994, ApJ, 433, L65 1996, ApJS, 106, 341

Mould, J. R., et al. 1991, ApJ, 383, 467

Noguchi, M. 1991, MNRAS, 251, 360

Norman, C., \& Ikeuchi, S. 1989, ApJ, 395, 372

Norman, C., \& Scoville, N. 1988, ApJ, 332, 124

Norris, R., et al. 1990, ApJ, 359, 291

Norris, R., \& Forbes, D. 1995, ApJ, 446, 594

Ohyama, Y., et al. 1999, ApJ, submitted (astro-ph/9903146)

Okumura S., et al. 1991, in IAU Symposium 146, Dynamics of Galaxies and their Molecular Distributions, ed. F. Combes (Dordrecht: Kluwer), 425

Osterbrock, D. 1989, Astrophysics of Gaseous Nebulae and Active Galactic Nuclei (Mill Valley, CA: Univ. Sci. Books)

Pagel, B., et al. 1979, MNRAS, 189, 75

Pastoriza, M., et al. 1993, MNRAS, 260, 177

Perry, J. 1992, in ASP Conf. Ser. 31, Relationships between AGN and Starburst Galaxies, ed. A. Filippenko (San Francisco: ASP), 169

Phillips, A. 1993, AJ, 105, 486

Plana, H., \& Boulesteix, J. 1996, A\&A, 307, 391

Prestwich, A., Joseph, R., \& Wrigth, G. 1994, ApJ, 422, 73
Rees, M. 1977, ARA\&A, 42, 471

Rieke, G., \& Low, F. 1972, ApJ, 176, L95 1975, ApJ, 197, 17

Rieke, G., et al. 1980, ApJ, 238, 24

1985, ApJ, 290, 116

Rigopoulou, D., et al. 1996, A\&A, 315, L125

Robert, C., Leitherer, C., \& Heckman, T. 1993, ApJ, 418, 749

Rubin, V., \& Ford, W. 1983, ApJ, 271, 556

Rubin, V., Graham, J., \& Kenney, J. 1992, ApJ, 394, L9

Sakamoto K., et al. 1999, ApJ, 514, 68

Sanders, D. B., Egami, E., Lípari, S., Mirabel, I., \& Soifer B. T. 1995, AJ, 110,1993

Sanders, D. B., \& Mirabel, F. 1996, ARA\&A, 34, 749

Sanders, D. B., Scoville, N., \& Soifer B. T. 1991, ApJ, 370, 158

Sanders, D. B., Soifer, B. T., Elias, J. H., Madore, B. F., Matthews, K., Neugebauer, G., \& Scoville, N. Z. 1988a, ApJ, 325, 74

Sanders, D. B., Soifer, B. T., Elias, J. H., Neugebauer, G., \& Matthews, K. 1988b, ApJ, 328, L35

Sargent, A., Sanders, D. B., \& Phillips, T. 1989, ApJ, 346, L9

Scalo, J. 1990, in Windows of Galaxies, ed. G. Fabbiano et al. (Dordrecht: Kluwer), 125

Scarrott, S. M., Draper, P. W., \& Stockdale, D. P. 1996, MNRAS, 279, 1325

Schweizer, F. 1980, ApJ, 237, 303

1982, ApJ, 252, 455

1986, Science, 231, 227

1990, in Dynamics and Interactions of Galaxies, ed. R. Wielen (Heidelberg: Springer), 60

Scott, D., et al. 1998, Nature, 394, 219

Scoville, N. Z., Sargent, A., Sanders, D., \& Soifer, B. 1991, ApJ, 366, L5

Scoville, N. Z., \& Soifer, B. T. 1991, in Massive Stars in Starbursts, ed. C. Leitherer, N. Walborn, T. M. Heckman, \& C. Norman (Cambridge: Cambridge Univ. Press), 233

Sekiguchi, K. \& Anderson, K. 1987, AJ, 94, 644

Sellmaier, F., et al. 1996, A\&A, 305, L37

Sersic, J. L. 1959, Z. Astrophys., 47, 9

Shaaf, R., et al. 1989, ApJ, 336, 762

Shaya, E., et al. 1994, AJ, 107, 1675

Shier, L., \& Fischer, J. 1998, ApJ, 497, 163

Shier, L., Rieke, M., \& Rieke, G. 1994, ApJ, 433, L9 1996, ApJ, 470, 222

Shlosman, I., \& Noguchi, M. 1993, ApJ, 414, 474

Smith, H., Lonsdale, C., Lonsdale, C., \& Diamond, P. 1998, ApJ, 493, L17

Stanford, S., \& Balcells, M. 1990, ApJ, 355, 59

Stone, R., \& Baldwin, J. 1983, MNRAS, 204, 357

Storchi-Bergmann, T., Kinney, A., \& Challis, P. 1995, ApJS, 98, 103

Storchi-Bergmann, T., Wilson, A., \& Baldwin, J. 1996, ApJ, 460, 252

Suchkov, A., Allen, R., \& Heckman, T. 1993, ApJ, 413, 542

Suchkov, A., Balsara, D., Heckman, T., \& Leitherer, C. 1994, ApJ, 430, 511

Suchkov, A., \& Berman, V. 1992, in Proc. Evolution of ISM and Dynamics of Galaxies, ed. J. Palous, W. Burton, \& P. Lindbland (Cambridge: Cambridge Univ. Press), 392

Suchkov, A., Berman, V., Heckman, T., \& Balsara, D. 1996, ApJ, 463, 528

Taniguchi, Y., Ikeuchi, S., \& Shioya, K. 1999, ApJ, 514, L9

Taniguchi, Y., \& Shioya, K. 1998, ApJ, 501, L67

Taniguchi, Y., Trentham, N., \& Shioya, K. 1998, ApJ, 504, L79

Taniguchi, Y., \& Wada, K. 1996, ApJ, 469, 581

Telesco, C., \& Gatley, I. 1984, ApJ, 284, 557

Tenorio-Tagle, G., \& Muñoz-Tuñon, C. 1997, ApJ, 478, 134

Terlevich, E. 1993, Rev. Mexicana Astron. Astrofis., 27, 29

Terlevich, E., Diaz, A., \& Terlevich, R. 1990, MNRAS, 242, 271

Terlevich, R., et al. 1991, A\&AS, 91, 285 1992, MNRAS, 255, 713

Tomisaka, K., \& Ikeuchi, S. 1988, ApJ, 330, 695

Toomre, A. 1977, in The Evolution of Galaxies and Stellar Population, ed.

B. Tinsley \& R. Larson (New Haven: Yale Univ. Obs.), 401

Toomre, A., \& Toomre, J. 1972, ApJ, 178, 623

Ulrich, M. H. 1972, ApJ, 178, 113

1978, ApJ, 219, 424

Veilleux, S., \& Osterbrock, D. E. 1987, ApJS, 63, 295

Veilleux, S., et al. 1994, ApJ, 433, 48 1996, ApJS, 98, 171

Veilleux, S., Kim, D., \& Sanders, D. 1999, ApJ, 522, 113

Vilches, J., et al. 1988, MNRAS, 235, 633

Weedman, D., et al. 1981, ApJ, 248, 105

Weil, M. L., \& Hernquist, L. 1996, ApJ, 460, 101

White, S. 1994, in Mass-Transfer Induced Activity in Galaxies, ed. I. Shlosman (Cambridge: Cambridge Univ. Press), 464

Whitmore, B., et al. 1999, AJ, 118, 1551

Williams, R., \& Perry, J. 1994, MNRAS, 269, 538

Wright, G., et al. 1988, MNRAS, 233, 1

Zenner, S., \& Lenzen, R. 1993, A\&AS, 101, 363

Zepf, S. 1993, ApJ, 407, 448

Zepf, S., Ashman, K., English, J., Freeman, K., \& Sharples, R. 1999, AJ, 118,752 